\title{
Les Hymenolepis de Charadriiformes
}

\section{(Seconde note à propos d'une vingtaine d'autres descriptions dont deux nouvelles)}

\author{
par S. DEBLOCK
}

\section{Plan}

A) Introduction.

B) Complément à la revue de 1962 des espèces connues et amendements de certaines descriptions :

1. Hymenolepis (Hym.) amphitricha (Rud., 1819).

2. Hymenolepis (Hym.) annandalei Southwell, 1922.

3. Hymenolepis (Echinocotyle) brachycephala (Creplin, 1829) nom. nov.

4. Hymenolepis (Hym.) capellae Baer, 1940.

5. Hymenolepis (Hym.) clandestina (Krabbe, 1869).

6. Hymenolepis (Hym.) glandularis Fuhrm., 1909.

7. Hymenolepis (Hym.) himantopodis (Krabbe, 1869).

8. Hymenopelis (Hym.) interrupta (Rud., 1802) Fuhrm., 1906.

9. Hymenolepis (Hym.) kwangensis (Southwell et Lake, 1939) nov. comb.

10. Hymenolepis (Echino.) longirostris (Rud., 1819) nov. comb.

11. Hymenolepis (Echino.) magnisaccis (Meggitt, 1927) nov. comb.

12. Hymenolepis (Hym.) nitidulans (Krabbe, 1882).

13. Hymenolepis (Hym.) porale Meggitt, 1927.

14. Hymenolepis (Hym.) recurvirostrae (Krabbe, 1869).

15. *Hymenolepis » recurvirostroides Meggitt, 1927.

16. Hymenolepis (Hym.) tsengi Joyeux et Baer, 1940.

17. Hymenolepis (Hym.) vaginata Baczynska, 1914.

C) Description d'espèces nouvelles :

1. H. (H.) aploparaksioidis n. sp.

2. H. (H.) rybickae $n . s p$. 


\section{D) Essai de clé diagnostique.}

E) Conclusion.

\section{A) INTRODUCTION}

Cette note complète notre révision antérieure des espèces d'Hymenolepis parasites de Charadriiformes, et résoud plusieurs des problèmes posés en 1962 (A.P.H.C., 1962, 37 [5-6], p. 767-847). Nous devons la plupart des solutions à l'extrême complaisance de M. le Professeur J. G. Baer qui a libéralement mis à notre disposition les très riches collections de l'Institut de Zoologie de Neuchâtel. L'examen de nombreuses préparations originales de Krabbe, Looss, Fuhrmann, Meggitt, Southwell, Joyeux, Baer... ne pouvait que combler notre curiosité ; nous renouvelons à $\mathrm{M}$. le Professeur Baer l'expression la plus sincère de nos remerciements et de notre gratitude pour l'accueil extrêmement cordial et sympathique qu'il nous a réservé dans son Institut. Nous remercions non moins chaleureusement M. S. Prudhoe, conservateur au British Museum pour l'empressement qu'il a mis à satisfaire sans réserve nos trop nombreuses demandes de matériel de référence concernant notamment les collections de Meggitt, ainsi que $\mathbf{M}^{\text {mes }}$ M. Voge de Los Angeles, K. Rybicka de Varsovie et M. Dubinina de Léningrad pour le prêt de préparations personnelles.

\section{B) COMPLEMENT A NOTRE REVUE DE 1962 DES ESPECES CONNUES ET AMENDEMENT DE CERTAINES DESCRIPTIONS}

\section{Remarques préliminaires :}

$1^{\circ}$ Les types ou les spécimens sans mention particulière d'origine proviennent de la collection de l'Institut de Zoologie de Neuchâtel. $2^{\circ}$ Certaines données d'hôtes ou de répartition géographique figurant déjà dans l'article de 1962 n'ont pas été répétées lorsqu'elles n'ont pas eu à subir de modifications importantes. $3^{\circ} \mathrm{La}$ terminologie et les abréviations utilisées sont les mêmes (cf. 1962, p. 768 et fig. 1 et 2). $4^{\circ}$ Toutes les figures ont été tracées à la chambre claire ; les échelles, sauf exception mentionnée, sont figurées en microns.

\section{1) HYMENOLEPIS (HYM.) AMPHITRICHA (Rud., 1819)}

Syn. : Hym. hamasigi (Yamag., 1940) Baer, 1962.

Nouvelles localisations venues à notre connaissance depuis 1962 : Mettrick, 1958 (Angleterre) ; Baer, 1962 (Islande).

L'homologie anatomique d'amphitricha et de hamasigi est manifeste ; les dissemblances de la taille et de la forme des crochets $(22-23 \mu \times 8$, de forme déliée pour la première espèce ; $33 \mu$, de forme plus massive pour la seconde) séparaient, à notre avis, les deux espèces. Néanmoins, l'exemple de $H$. clandestina (Krabbe) - dont les crochets 
présentent des différences du même ordre (cf. p. 700) — prouve qu'elles sont possibles chez une espèce donnée. De toute façon, la systématique actuelle, construite sur des bases morphologiques, se révèle incapable de résoudre ces difficultés; la biologie apportera peut-être une solution définitive. Nous accepterons donc la synonymie ci-dessus.

\section{2) HYMENOLEPIS (HYM.) ANNANDALEI Southwell, 1922}

nec Hym. annandalei var. longosacco Joyeux et Baer, 1939.

nec Hym. annandalei sensu Joyeux et Baer, 1940.

Matériel observé : $1^{\circ}$ types de Southwell ; $2^{\circ}$ et $3^{\circ}$ spécimens de Joyeux et Baer, 1939 et 1940.

Complément de description de l'espèce (planche 1, fig. 1 à 4).

SCOLEX et CROCHETS : bien figurés par Southwell.

Atrium génital : dépourvu de sacculus, inerme, tubulaire, long de 35-40 $\mu$.

Musculature longitudinale interne formée d'environ 45 bandes sur chaque face (fig. 3).

C.O.R. : dépourvus d'anastomose transverse.

Cou : long, $1.400 \times 110 \mu$ de diamètre. L'aspect de l'anneau mûr est celui que nous figurons (fig. 2).

\section{Appareil Reproducteur.}

\section{A) Système mâle.}

Testicules: du type B. $180 \times 90 \mu$ de diamètre à maturité.

V.S.E. : dans le prolongement de la P. du C. Piriforme. $100 \mu$ de long environ $\times$ $50 \mu$ de diamètre.

\section{B) Système femelle.}

OVAIRE : mûr à contours multilobés, 8 à 10 lobes, principalement antérieurs.

310 à $370 \mu$ de large $\times 140-180$, et même $200 \mu$, de hauteur ; échancré postérieurement au niveau de l'emplacement du vitellogène.

Vitellogène : 110-140 $\mu$ de large $\times 90 \mu$ de hauteur. Bord postérieur plus ou moins festonné.

R.S. : volumineux. $320 \mu \times 45 \mu$ de diamètre ; piriforme, il franchit les C.O.R. poraux pour s'unir au vagin.

VAGIN : nettement figuré, tubulaire, long de $90 \mu$ environ $\times 10-12 \mu$ de diamètre, à parois musculeuses épaisses de 4-5 $\mu$. Dépourvu de sphincter et de fibres rétractrices (fig. 4). 


\section{Discussion.}

Contrairement à la description et à l'illustration erronée de Southwell, la morphologie d'Hymenolepis annandalei ne s'éloigne pas du schéma classique ; elle s'apparente à celle de $H$. hughesi Webster, 1947. Hymenolepis annandalei longosacco Joyeux et Baer, 1939 est une espèce distincte que nous considérons synonyme de $H$. clandestina Krabbe (cf. la redescription plus loin). L'Hymenolepis annandalei sensu Joyeux et Baer, 1940 d'un Scolopax rusticola L. de Corse, est constitué d'un mélange de deux espèces différentes, appartenant chacune au genre Aploparaksis.

\section{3) HYMENOLEPIS (ECHINOCOTYLE) BRACHYCEPHALA}

(Creplin, 1829), nov. comb.

nec Hymenolepis (Hymenolepis) b. (Creplin, 1829) Railliet, 1839.

Syn. : Echinocotyle uralensis Clerc, 1902-1903.

Echinocotyle uralensis Clerc, 1902, sensu Southwell, 1922, pro parte.

Hymenolepis oweni Moghe, 1933.

Echinocotyle oweni (Moghe, 1933), sensu Macko, 1962.

Echinocotyle hypoleuci Singh, 1952.

Hymenolepis quasioweni Dubinina, 1953.

Hymenolepis sp. inq., $\mathrm{n}^{\circ}$ 2, Deblock et Rosé, 1962.

Région paléarctique: Europe occidentale, centrale et orientale. Russie d'Asie (Sibérie occidentale).

Région indienne: Indes (Moghe, Singh).

Matériel observé :

$1^{\circ}$ Exemplaires de Joyeux et Baer, 1936 (Dombes, France). (PI. 2, fig. 5 à 9).

$2^{\circ}$ Exemplaires de Southwell, 1922.

$3^{\circ}$ E. uralensis de Tringa glareola (16-7-36) et Tringa nebularia (13-8-1937), Sibérie occidentale.

$4^{\circ}$ H. quasioweni Dubinina, 1953. Types. Philomachus pugnax (9-8-1937) et (14-8-37), Sibérie occidentale.

Les préparations des lots 3 et 4 des collections de Léningrad nous ont été communiquées par Mme M. N. Dubinina.

En 1936, Joyeux et Baer (p. 315) signalent la présence d'Hymenolepis brachycephala (Creplin) chez le «combattant » de la région de Dijon ; nous en avons étudié les préparations: il s'agit d'un Echinocotyle typique; les anneaux, malgré une forte contracture qui ramène les trois testicules en ligne, sont morphologiquement tout à fait identiques à l'espèce que nous avions figurée et décrite en 1962 sous le nom d'Hymenolepis sp. inq. $\mathrm{n}^{\circ} 2$, p. 836.

Ses caractéristiques sont les suivantes: taille de 2 à $3 \mathrm{~cm} \times 1$ à $1,5 \mathrm{~mm}$ de large ; atrium génital garni d'une couronne d'épines nombreuses longues de 5 à $6 \mu$ et muni 
d'un sac accessoire dorsal de 30-40 $\times 20-25 \mu$ de diamètre à épines de $2,5 \mu$. Il y a quatre B.M.I. (1). Scolex de $150 \times 160 \times 90 \mu$; ventouses ovalaires de $90 \mu$ de grand diamètre ; sac du rostre bien développé de $140 \times 50 \mu$. Une couronne de 10 crochets claviformes de 56-63 $\mu$, dont le manche est deux fois plus long que la lame. Trois testicules disposés en ligne. Ovaire postérieur, large, dépassant latéralement les C.O.R. Vitellogène disposé dorsalement par rapport à l'ovaire. Poche du cirre courte de 150 à $200 \mu$ de long. $\mathrm{PC} / \mathrm{AM}=1 / 6$ à $1 / 8$. Cirre glabre de $40 \mu$ environ. Eufs (imparfaitement mûrs) nettement ovalaires; membrane externe 46-56 $30 \mu$; membrane interne $24 \times 13 \mu$; embryon hexacanthe $20-22 \times 10 \mu$; crochets de $7 \mu$ environ.

En 1962, J. K. Macko décrit et figure en détail une espèce d'Echinocotyle d'Europe Centrale (Slovaquie), parasite de plusieurs hôtes: Larus ridibundus L., Anas crecca L. et Philomachus pugnax L. Identifiée à Hymenolepis oweni Moghe, 1933, l'espèce est mise en synonymie avec $H$. quasioweni Dubinina, 1953. La description de l'auteur coïncide assez exactement avec chacun des différents matériels que nous avons étudiés (2). L'examen des types de $H$. quasioweni montre, en effet, que la spinulation ventousaire de ce Cestode est celle d'un Echinocotyle classique (une vingtaine d'éventails disposés sur le bord muscu'eux, comptant chacun de 1 à 5 épines de 6 à $7 \mu$ de long, suivant leur emplacement ; sur le fond, il s'y ajoute une douzaine de rangs verticaux d'épines identiques). Echinocotyle hypoleuci Singh, 1952, ne se distingue de $H$. brachycephala par aucun caractère essentiel, ni non plus $E$. uralensis Clerc. Cette dernière synonymie est confirmée, point par point, par l'anatomie tout entière des exemplaires sibériens de $E$. uralensis que nous avons examinés.

Il se trouve en conséquence que le seul nom valable de l'ensemb.e de ces Cestodes soit celui proposé par Creplin dès 1829. Echinocotyle uralensis sensu Southwell, 1922, représente vraisemblablement un mélange de deux espèces différentes comme le soupçonnait l'auteur. Les crochets de $78 \mu$ sont d'une silhouette très différente de celle de brachycephala, se rapprochant de celle de type nitida; les préparations ne permettent pas une identification plus précise.

\section{4) HYMENOLEPIS (HYM.) CAPELLAE Baer, 1940}

Matériel observé : types. (planche 3, fig. 10 et 11).

\section{Complément de description :}

Dix crochets de $23-24 \times 12,5 \mu$ de hauteur ; $16 \mu$ à la base. Testicules du type B, rarement du type $\mathrm{C}$ et jamais du type $\mathrm{G}$. Poche du cirre à parois musculeuses relativement épaisses. Cirre évaginé : $140 \mu$ de long $\times 15 \mu$ de diamètre moyen, régulièrement

(1) Le nombre des B.M.I. fixé à 15-20 est une erreur d'observation dont nous nous excusons, due à l'excessive décontraction de nos exemplaires qui nous a fait probablement confondre les bandes musculaires longitudinales internes et externes.

(2) Selon Macko, l'œuf mûr de l'espèce est ovale et mesure $60-80 \times 60-68 \mu$ de diamètre ; sa membrane externe est finement grenue; la membrane interne mesure $30-35 \times 22 u$ et les crochets de l'embryon 9 à $11 \mu$. 
cylindrique, légèrement massué à son extrémité où le diamètre atteint 20-22 $\mu$; garni sur la totalité de sa longueur de soies de $5 \mu$ de long dont la taille s'atténue progressivement pour ne plus être représentées que par des points sur le renflement terminal (fig. 11).

Canal déférent court ne dessinant qu'une courte boucle porale lorsque le cirre est invaginé ; au niveau de son raccord avec la V.S.I., il s'entoure d'une série de fibres musculaires annulaires assez bien visibles.

Vagin distal (copulateur) conique, strié longitudinalement par des plicatures de sa paroi ; long de $80-100 \times 17 \mu$ de diamètre maximal au niveau de l'atrium génital. Dépourvu de sphincter et de fibres rétractrices; le vagin proximal demeure invisible. R.S. ovoïde, médian, ne dépassant pas les C.O.R. poraux. Ovaire bilobé, de $210 \times 90 \mu$ à maturité et à bords légèrement festonés. La concavité postérieure, très échancrée, contient le vitellogène de $45 \times 68 \mu$.

L'utérus mûr présente une tendance nette à la bilobation ; œufs nombreux, ovoïdes de $40 \times 33 \mu$, à coque interne légèrement épaissie.

\section{5) HYMENOLEPIS (HYM.) CLANDESTINA (Krabbe, 1869)}

Syn. : Hymenolepis annandalei longosacco Joyeux et Baer, 1939.

Région paléarctique : Europe (Grande-Bretagne, France).

Matériel observé : type de Joyeux et Baer.

Hôte: Tringa sp. (environs de Marseille, France).

Redescription de l'espèce. 'Planche 4, fig. 12 à 15).

Strobile : long de plusieurs centimères. Formé de très nombreux anneaux.

PORES GÉNITAuX : unilatéraux dextres.

Atrium génital: dépourvu de sacculus et d'épines.

Musculature longitudinale externe formée d'une quarantaine de faisceaux; l'interne est formée d'une trentaine de faisceaux volumineux sur chaque face ; ils semblent issus de $2 \times 3$ faisceaux très forts s'insérant à la périphérie du sac du rostre.

C.O.R. longitudinaux non anastomosés, ventraux par rapport aux conduits génitaux.

Scolex : rappelle morphologiquement celui de $H$. hughesi var. europaei, mais avec un sac du rostre plus développé ; de forme générale massuée ; peu différencié du cou. Taille : $200-250 \times 250 \mu$ de diamètre. Ventouses sphériques ou circulaires, musculeuses, inermes, de $80 \mu$ de diamètre. Sac du rostre : 150-200 $\times 80-95 \mu$ de diamètre, dépassant plus ou moins largement le bord postérieur des ventouses. Rostre évaginé : 280-300 $\mu$ jusqu'au fond du sac $\times 70 \mu$ de diamètre, la portion évaginable étant de 100 à $150 \mu$ (fig. 12).

Une couronne de 10 crochets chéliformes assez massifs de 28-30 × 11-13 $\mu$, la base, proiongée d'un manche court bien dessiné, mesurant 22-25 $\mu$ (fig. 13).

Cou : de $180 \mu$ de diamètre. Peu marqué. 
ANNEAUX : trapézoïdaux beaucoup plus larges que haut ; testicules persistants dans les anneaux femelles.

\section{Appareil reproducteur.}

\section{A) Système mâle.}

Testicules: en ligne (type E), les glandes femelles naissant d'un côté ou de l'autre du testicule médian. Taille: $90 \mu$ de diamètre (fig. 14).

V.S.E. : rarement observée à réplétion. Ovoïde : $70 \mu$ de diamètre, située dans le prolongement de la poche du cirre, en avant du lobe antiporal de l'ovaire.

P. du C. : fusiforme. Bien développée : 390-410 $\times 56 \mu$ de diamètre (dans un anneau mûr de $780 \times 125 \mu$ ), atteignant presque les C.O.R. antiporaux dans les anneaux mûrs. Rapport PC/AM = 1/2 (1). Parois musculeuses épaisses.

CIRre évaginé cylindrique long et fin: $120-140 \mu$ (jusqu'à $180 \mu$ ) $\times 10-11 \mu$ à la base et $8 \mu$ à l'extrémité (fig. 15).

\section{B) Système femelle.}

OVAIRE : masse indivise, médiane et ventrale, de $230 \times 90-100 \mu$ située légèrement en arrière des testicules.

Vitellogène: situé légèrement en arrière de l'ovaire; 90-100 × 35-45 $\mu$ de haut.

R.S. : ovoïde ou sphérique, de 90 à $110 \mu$ de diamètre situé en avant de l'aile porale de l'ovaire, ne dépassant pas les C.O.R. poraux.

VAGIN proximal membraneux, tubulaire, dépourvu de sphincter et de fibres rétractrices. Long de $110 \mu \times 7-8 \mu$ de diamètre.

UTÉrus gravide jeune bisacculé.

EUFs inconnus.

\section{Discussion.}

Jusqu'à présent les crochets de $H$. clandestina étaient tenus pour mesurer de 40 à $47 \mu$. Les crochets des spécimens décrits ci-dessus sont nettement plus courts (28-30 $\mu)$; en dépit de cette différence de taille, on peut considérer l'espèce de Joyeux et Baer identique à celle de Krabbe par le reste de sa morphologie (poche du cirre et conduits génitaux notamment), la position de l'ovaire par rapport aux testicules ayant pu être modifiée par la contraction du strobile dans le spécimen de Davies.

N. B. - Hymenolepis uliginosa possède des crochets de morphologie et de taille fort voisines $(42-45 \mu)$, mais un strobile de $4 \mathrm{~mm}$ seulement de long $\times 0,250$.

6) HYMENOLEPIS (HYM.) GLANDULARIS Fuhrm., 1909

$=$ Orlovilepis g. (Fuhrm., 1909) Spassky et Spasskaïa, 1954.

Cloacotaenia g. (Fuhrm., 1909) Yamaguti, 1959.

(1) Ce rapport égale 1/3 dans les anneaux mâles. 
Matériel observé : types représentés par un scolex in toto et des coupes histologiques longitudinales et transverses de strobiles complets très contractés.

Complément de description de l'espèce-type (planche 5, fig. 16 à 21).

Strobiles courts $3-4 \mathrm{~mm}$, de 0,70 à $0,75 \mathrm{~mm}$ de largeur maximale au niveau des anneaux mûrs. Cou bien marqué de $400 \mu$ de diamètre. Plusieurs scolex ne mesurent que $820 \mu$ de diamètre $\times 450 \mu$ de hauteur (fig. 16) ; ventouses de $280 \mu$ de diamètre ; inermes. Sac du rostre de $230 \times 90 \mu$ de diamètre; rostre absent. Le sac du rostre, entièrement clos, ne contient qu'un amas cellulaire. La partie antérieure du scolex ne porte pas l'orifice habituel permettant l'évagination du rostre.

Musculature interne assez puissante formée de 20 à 30 B.M.I. par face, de section lenticulaire (fig. 17).

Poche DU CIRRE (fig. 20 et 21). N'atteint pas les C.O.R. antiporaux, mais seulement le milieu de l'anneau. Piriforme, allongée : $320 \times 40 \mu$. Parois musculaires minces. V.S.I. de $80 \times 45-50 \mu$. Cirre de $12 \mu$ de diamètre, puissamment armé de fortes soies de $6 \mu$ de long, sur une longueur de 100-110 $\mu$; non observé évaginé. Le canal déférent qui lui fait suite semble pourvu de villosités bien marquées. V.S.E. non observable.

Testicules de $80 \mu \times 45 \mu$ de hauteur, en ligne sur les exemplaires contractés (fig. 18).

OVAIRE : petit, $150 \mu$ de large, médian.

V AGIN : long de $70 \mu$ environ $\times 18-20 \mu$ de diamètre ; entouré de cellules portant son diamètre externe à $30 \mu$; lumière garnie de papilles de $5 \mu$ de haut, analogues à celles qui tapissent le déférent. Le vagin communique par une large ouverture avec un R.S. trapu, ovoïde, de $55 \times 35 \mu$, situé au niveau ou au-delà des C.O.R. poraux.

L'œuF non encore mûr (l'embryon hexacante étant dépourvu de crochets), est sphérique et mesure $37 \mu$ de diamètre ; il est néanmoins déjà entouré d'une enveloppe nettement épaissie de 1,5 à $2 \mu$ formant coque résistante (fig. 19).

\section{7) HYMENOLEPIS (HYM.) HIMANTOPODIS (Krabbe, 1869)}

nec Hymenolepis himantopodis sensu Meggitt, 1927.

Hymenolepis himantopodis sensu Shen Tseng, 1933.

Hymenolepis himantopodis sensu Baer, 1940.

Hymenolepis himantopodis sensu Singh, 1959.

Hymenolepis $(H$.$) recurvirostrae sensu Baer, 1959$.

Matériel observé : types constitués des exemplaires de Rudolphi (coupes transversales d'anneaux gravides et longitudinales d'anneaux mûrs), ayant servi à l'étude de Fuhrmann en 1906 (2).

Complément de description de l'espèce-type (planche 6, fig. 22-23)

Strobile (suivant Krabbe, 1869): $50 \times 1 \mathrm{~mm}$.

(2) Nous avions omis de prendre connaissance de celle-ci en 1962. 
ANNEAU MûR : $800 \times 75 \mu$ de haut ; anneau gravide jeune : $900 \times 400 \mu$ d'épaisseur.

Musculature longitudinale: sur chaque face 40 à 50 B.M.E. très fines et une quinzaine de B.M.I. de 10-20 $\mu$ de diamètre.

Atrium GÉnital : tubulaire, long de $100 \mu$, dépourvu de sacculus, d'épines et de toute ornementation. Les conduits génitaux des deux sexes sont seuls observables sur les préparations.

\section{A) Appareil mâle.}

Poche DU CIRre : $260-275 \times 55-60 \mu$; dorsale, atteignant le milieu de l'anneau ou le dépassant légèrement ; parois fortement musculeuses; pourvue de quelques fines fibres rétractrices s'insérant au niveau de son fond. V.S.I. située dans la moitié postérieure, se poursuivant en avant par un canal déférent qui décrit une courte boucle ; absence de tube chitinoïde. CIRre observé invaginé ou très légèrement évaginé ; court : 35-40 $\mu \times 6-10 \mu$ de diamètre, finement ponctué. V.S.E. située dans le prolongement de la poche du cirre, ovoïde, $100 \mu$ de diamètre $\times 125 \mu$ de long. Canal intervésiculaire de $100 \mu$ environ.

\section{B) Appareil femelle.}

VAGIN distal de 100-110 $\mu$, ventral, chitinoïde, cylindro-conique, dépourvu de papilles ; occlus par un sphincter bien figuré de $20 \mu$ de diamètre, et coudé à $90^{\circ}$ par un volumineux faisceau de fibres rétractrices de 15-20 $\mu$ de diamètre allant s'insérer au niveau du bord antiporal de l'anneau. Vagin proximal en forme de tube membraneux de $75 \mu$ de long. R.S. de $150 \times 50 \mu$, ovoïde, ventral, ne franchissant pas les C.O.R. poraux.

\section{Discussion.}

Malgré ses lacunes, cette description établit que l'espèce de Krabbe (caractérisée par son cirre court de $40 \mu$ ponctué, son atrium tubulaire dépourvu d'ornementation, son vagin de structure simple, ses 15 B.M.I.) n'a jamais été retrouvée. H. himantopodis sensu Meggitt, 1927 s'identifie à $H$. (H.) recurvirostrae (Krabbe, 1869), tandis qu'au sens de Shen Tseng, 1933, à celui de Baer, 1940 et à celui de Singh, 1959, les Cestodes s'identifient au contraire à $H$. $(H$.) tsengi Joyeux et Baer, 1940 (cf. plus loin).

8) HYMENOLEPIS (HYM.) INTERRUPTA (Rud., 1802) Fuhrm., 1906

Syn. : Hym. styloides Fuhrm., 1906.

Hym. styloides Fuhrm., 1906 sensu Joyeux et Baer, 1935.

$=$ Weinlandia styloides (Fuhrm., 1906) Shen Tseng, 1933.

Hym. clerci (Shen Tseng, 1933).

$=$ Fuhrmaniella clerci Shen Tseng, 1933.

H. shentsengi (Shen Tseng, 1933) Hughes, 1940.

Dicranotaenia visigi (Shen Tseng, 1933) Skriabine et Mathevossian, 1942. 
Hym. clerci (Shen Tseng, 1933) sensu Tubangui et Masiluñgan, 1937.

Hym. fola Meggitt, 1933.

Oligorchis toxometra Joyeux et Baer, 1928.

Hym. spinosa Linstow, 1906.

Hym. spinosa Linstow, 1906 sensu Southwell et Lake, 1939.

Région paléarctique: Afrique (Egypte, Congo, Afrique Occidentale); Chine.

Région indienne: (Indes, Ceylan, Philippines).

Matériel observé : $1^{\circ}$ types de Fuhrmann. $2^{\circ}$ types de Shen Tseng, 1933. $3^{\circ}$ types de Joyeux et Baer, 1928. $4^{\circ}$ Exemplaires de Joyeux et Baer, 1935 (Tonkin).

Nous résumons ci-dessous la fiche d'identification de ce Cestode caractéristique, essentiellement d'après l'observation des exemplaires de Shen Tseng. Cependant, l'ensemble du matériel examiné, bien que de provenances fort diverses, montre une très grande homogénéité morphologique.

\section{Redescription de l'espèce (planche 7, fig. 24 à 27)}

Strobile : longueur totale : $60-100 \mathrm{~mm}$ sur $0,700-0,800 \mathrm{~mm}$ de largeur maximale.

PORES GÉnITAUX : unilatéraux dextres.

ATRIUM GÉNitAL : dépourvu de sacculus et d'épines. 50 à $60 \mu$ de profondeur.

Musculature longitudinale interne, formée de 4 faisceaux; externe, formée d'une trentaine de faisceaux sur chacune des deux faces. Enserrant étroitement les glandes génitales.

C.O.R. : ventraux par rapport aux conduits sexuels et dépourvus d'anastomose transverse.

Scolex : taille : $215 \mu$ de diamètre $\times 230 \mu$ de long environ. Ventouses : $90 \mu$ de diamètre, inermes. Rostre évaginé : $225 \mu$ de long $\times 45 \mu$ de diamètre maximal. Sac du rostre : $110 \mu$ de long $\times 45 \mu$. Une couronne de 10 crochets claviformes de 32-35 $\mu \times 5$ à $6 \mu$ de hauteur, à manche environ 5 fois plus long que la lame (fig. 24).

Cou : court, de $100 \mu$ de diamètre, la strobilisation débutant rapidement.

ANNEAUX Mûrs : trapézoïdaux. $280 \mu$ de hauteur $\times 780$ de largeur à la base et $520 \mu$ au sommet (fig. 25).

\section{Appareil reproducteur.}

\section{A) Système mâle.}

Testicules: constamment en position du type D. 120-140 $\mu \times 70-90 \mu$ de hauteur. Certains strobiles montrent une protérandrie marquée (testicules disparus dans les anneaux femelles mûrs, juste avant la ponte ovulaire) ; elle ne paraît pas constante.

POCHE DU CIRRE : très développée, de 350 à $380 \mu \times 45-55 \mu$ de diamètre, presque toujours en forme d'accent circonflexe ; atteint ou dépasse légèrement l'axe longi- 
tudinal du strobile. Sa paroi, épaisse de 6 à $10 \mu$, est formée de fortes fibres musculaires longitudinales. $\mathrm{PC} / \mathrm{AM}=1 / 2$.

V.S.I. située dans la moitié ou les $2 / 3$ postérieurs de la poche du cirre ; elle se continue par un canal déférent très long plus ou moins pelotonné, différencié en un mince tube chitinoïde.

CIRre très long à l'état d'évagination: $260 \mu \times 20-25 \mu$ à la base et 10-14 $\mu$ à l'extrémité ; garni sur toute sa longueur d'un revêtement dense de fines soies de $5 \mu$ de long. Dans certains anneaux, le cirre soyeux sort d'un étui, parfois long de plus de $100 \mu$, dépourvu d'épine, qui représente probablement la base glabre d'un cirre ayant subi une double invagination à l'intérieur de la poche du cirre.

VÉSicule SÉminale EXTERNE: ovoïde, située dorsalement au-dessus de la portion antiporale de la poche du cirre. Volumineuse : 180-200 $\times 140-120 \mu$.

\section{B) Système femelle.}

OvaIRE : médian, ventral, souvent bilobé en forme de haricot, à concavité postérieure, les lobes subissant eux-mêmes une lobation secondaire plus ou moins accentuée ; $330 \times 70-80$ à $100 \mu$ de haut.

Vitellogène : entier ou bilobé : 70-90 × 45-60 $\mu$. Postérieur à l'isthme ovarien.

R.S. : symétrique de la V.S.E. par rapport à l'axe longitudinal, antérieur et ventral. Parfois observé dorsal à la poche du cirre. A peu près de même volume que la V.S.E. :

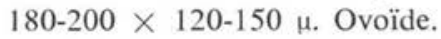

VAGIN : uniquement membraneux, tubulaire. Ventral par rapport à la P. du C. Du R.S. naît un tube membraneux étroit de $190 \mu$ de long $\times 6 \mu$ de diamètre interne et $16 \mu$ de diamètre externe, très sinueux ; la portion distale, de parois minces non chitinoïdes mesure $80 \mu \times 17 \mu$ de diamètre interne et $25 \mu$ de diamètre externe ; entourée de fines fibres musculaires annulaires peu apparentes, et faiblement lignée longitudinalement. Absence de sphincter et de fibres rétractrices (fig. 26).

\section{Discussion.}

Les crochets du rostre de $H$. interrupta (Rud., 1802) et de $H$. fola Meggitt, 1933, ainsi que la taille de la poche du cirre, constituent à peu près les seuls critères d'identification de ces Cestodes qui ne semblent pas pouvoir être discriminés de l'espèce décrite. L'identité est certaine en ce qui concerne $H$. fola sensu Yamaguti et fort probable pour H. spinosa Linstow, 1906 (identité d'hôte et de répartition géographique, en plus des caractères morphologiques ne prêtant pas à confusion). La description de Southwell et Lake, 1939 (chez Rostratula benghalensis du Congo belge) ne s'en écarte guère non plus. Dans le cas d'Oligorchis toxometra, il apparaît que les auteurs semblent avoir confondu, sur les coupes histologiques des anneaux mâles, le massif des glandes femelles avec un testicule surnuméraire ; aucun anneau monté in toto ne présente un nombre de ces organes différent de trois. Il y a bien 4 B.M.I. et non 6 par face.

En conséquence, nous croyons justifié et raisonnable de faire tomber toutes les espèces ci-dessus en synonymie avec $H$. $(H$.$) interrupta (Rud., 1802) Fuhrm., 1906$ la plus anciennement signalée. 


\section{9) HYMENOLEPIS (HYM.) KW ANGENSIS}

(Southwell et Lake, 1939) nov. comb.

Syn. : Oligorchis $k$. Southwell et Lake, 1939.

Hybridolepis $k$. (Southwell et Lake, 1939) Spassky, 1959.

nec Hymenolepis recurvirostrae sensu Baer, 1959 (p. 103).

Région paléarctique: Congo ex-Belge (Kwango).

Hôte: Galachrisia nuchalis (Gray).

Matériel observé : type composé de plusieurs scolex isolés ou attenants à un fragment de strobile comportant des anneaux mâles difficilement lisibles, et d'anneaux mûrs et gravides immatures détachés et montés à plat.

Redescription de l'espèce-type (planche 8, fig. 28 à 32)

Strobile : au moins $10 \times 1,5 \mathrm{~mm}$; la longueur totale devrait atteindre le double sinon davantage.

PORES GÉNITAuX : unilatéraux, dextres.

ATRIUM GÉNITAL: tubulaire, relativement long sur les anneaux contractés (130-150 $\times$ 20-25 $\mu$ de diamètre), entouré de cellules glandulaires. Dans son fond et en contact intime avec l'extrémité porale de la poche du cirre (3) se situe un «peigne atrial » nettement figuré de 10-13 $\mu$ de diamètre, garni intérieurement de soies de 5 à $6 \mu$ et extérieurement d'un massif glandulaire de 25-50 × 10-25 $\mu$ de diamètre (fig. 31).

Musculature longitudinale externe formée de 40 fins faisceaux environ, la musculature longitudinale interne de 9 faisceaux plus volumineux.

C.O.R. ventraux dépourvus d'anastomose transverse.

Scolex : taille : $120 \mu$ de long $\times 150-200 \mu$ de diamètre. Ventouses glabres : $75 \mu$ de diamètre. Sac du rostre : $90 \mu \times 60-75 \mu$ de diamètre dépassant légèrement le bord postérieur des ventouses. Rostre évaginé court et globuleux : $30 \times 30 \mu$ de diamètre (fig. 28).

Crochets : $1 . X .8$ (et non pas $12 \mu$ d'après les auteurs) $\times 5,5 \mu$ de hauteur, chéliformes, à lame légèrement plus longue que la garde. Cou: $120 \mu$ de diamètre.

AnNEAux : trapézoïdaux (fig. 29).

\section{Appareil reproducteur.}

\section{A) Système mâle.}

Testicules : difficilement visibles, les anneaux mâles étant assez épais et contractés. La protérandrie est accusée et les testicules ne persistent pas dans les anneaux femelles mûrs plus lisibles. Leur nombre ne semble pas devoir dépasser trois ; certains

(3) La juxtaposition étroite de ces deux formations pourrait faire croire que le peigne atrial est inclus à l'intérieur de la poctie du cirre. 
anneaux n'en présentent apparemment que deux. Disposés en ligne, ils mesurent 20-25 $\times$ 40-50 $\mu$ (dans des anneaux de $400 \mu$ de large $\times 25 \mu$ de haut).

POCHE DU CIRRE : dans les anneaux mâles jeunes, la poche du cirre atteint toujours le milieu de l'anneau franchissant dorsalement les C.O.R. poraux. Dans les anneaux mûrs, elle ne l'atteint que plus rarement, mesurant 180-200 $\mu \times 45-50 \mu$ de diamètre. Elle se situe assez profondément dans l'anneau par suite de l'allongement de l'atrium génital. Rapport $\mathrm{PC} / \mathrm{AM}=1 / 3$. Parois musculeuses d'épaisseur moyenne $(5 \mu)$. Présence de fibres rétractrices musculaires très fines insérées isolément à la partie antérieure de la poche du cirre (fig. 31).

Crrre : à l'état invaginé, se présente sous forme d'un tube de $6 \mu$ de diamètre, à lumière tapissée de très fines épines sur une longueur de $20 \mu$. A l'état d'évagination la plus grande que nous avons pu observer, il est court, cylindro-conique, finement armé, mesurant 20 à $30 \mu$ de long $\times 8,5 \mu$ de diamètre basal. Il ne semble pas $\mathrm{y}$ avoir de différenciation chitinoïde du canal éjaculateur (fig. 30 et 31).

Vésicule séminale eXterne : située dorsalement dans le prolongement de la poche du cirre. Ovoïde, ou piriforme lorsqu'elle est étirée par le canal intervésiculaire ; mesure de $50 \times 80 \mu$ à $200 \times 100 \mu$ de diamètre.

\section{B) Système génital femelle.}

OVAIRE : généralement trilobé, médian, ne dépassant latéralement pas les C.O.R. Taille : de $120 \times 80 \mu$ de haut à $320 \times 40 \mu$.

Utérus jeune tubulaire, dépassant les C.O.R.

RÉCEPTACLE SÉminal ovoïde ne dépassant pas les C.O.R. poraux. Situé au niveau de l'aile porale de l'ovaire, postérieurement à la poche du cirre. Taille identique à celle de la vésicule séminale externe.

VAGIN : de structure simple, tubulaire, ventral par rapport à la poche du cirre. Portion copulatrice peu différenciée, apparemment non chitinoïde, dépourvue de papille, de sphincter et de fibres rétractrices.

UTÉRUS : sacculaire envahissant la totalité du parenchyme médullaire; œufs mûrs inconnus.

\section{Discussion.}

En dépit d'un examen attentif, l'impossibilité de mettre en évidence dans les anneaux mâles des préparations-types un nombre de testicules supérieur à trois, nous incite avec Baer, 1959 à déplacer ce Cestode décrit comme Oligorchis dans le genre Hymenolepis (Hymenolepis). Il apparaît que les auteurs à qui la protérandrie du Cestode semble avoir échappé se soient efforcés de distinguer des testicules dans les anneaux femelles qui n'en comportaient plus (cf. leur fig. 5).

La présence d'un peigne atrial, le nombre différent des B.M.I., entre autres caractéristiques, différencient aisément cette espèce de H. recurvirostrae (Krabbe, 1869). 


\section{IU) HYMENOLEPIS (ECHINO.) LONGIROSTRIS}

(Rud., 1819) nov. comb.

nec Hymenolepis (Hym.) l. (Rud., 1819) Railliet, 1899.

Syn.: Hymenolepis multiglandularis Baczynska, 1914.

Région paléarctique : Europe (Italie, France); Afrique (Congo, Egypte).

Hôtes: Glareola pratincola (L.), Philomachus pugnax (L.), Charadrius pecuarius, Larus fuscus $\mathrm{L}$.

Matériel observé : $1^{\circ}$ exemplaires de Baer, 1956 ( $P$. pugnax, France, Dombes) constitués d'une dizaine de strobiles contractés et de coupes histologiques longitudinales et transverses. $2^{\circ}$ Exemplaires de Baer, 1959 (C. pecuarius: Congo, Ishango). $3^{\circ}$ Exemplaires de H. multiglandularis Baczynska, 1914 sensu Meggitt, 1927.

\section{Redescription de l'espèce (planche 9, fig. 33 à 37)}

Strobile: de petite taille: 1,2-1,4 $\mathrm{mm}$ de longueur, mais sans anneaux gravides mûrs, $\times 0,25$ à $0,35 \mathrm{~mm}$ de largeur maximale. Nombre d'anneaux peu élevé : 50 à 60 y compris ceux du cou; ils se décomposent environ comme suit : 25 anneaux de cou et immatures, 15 anneaux sexuellement mûrs, 10 anneaux gravides immatures.

PORES GÉNITAUX : unilatéraux dextres.

Atrium génital : court. Garni d'une couronne d'épines de $6 \mu$ de long entourant le seul orifice mâle et d'un sac accessoire dorsal, bien développé de 65-75 $\times$ 30-36 $\mu$ de diamètre; sa lumière longue de $45 \mu$, est garnie d'épines fines de $3 \mu$ de long. Il est entouré d'un important massif glandulaire.

Musculature longitudinale: externe, formée de 30 à 40 fibres simples; interne, formée de 4 faisceaux fins ( $6 \mu$ de diamètre).

Scolex : petit, pyramidal, $130-150 \mu$ de long $\times 110 \mu$ de diamètre ; les quatre ventouses sont typiques du sous-genre Echinocotyle, spinulées (13 à 15 rangs de une à 3 ou 4 petites épines) et mesurent $70 \times 45 \mu$. Sac du rostre allongé dépassant nettement le bord postérieur des ventouses et pénétrant parfois dans les premiers anneaux de cou : $130 \times 40 \mu$ de diamètre. Rostre non observé évaginé. Garni d'une couronne de 10 crochets falciformes de $35 \mu$, la lame mesurant $15 \mu$ et le manche $20 \mu$ de longueur (fig. 33 et 34 ).

Cou : très court, large de $100 \mu$ sur les exemplaires contractés; plus nettement figuré sur les exemplaires normaux (cf. fig. 33).

AnNEAuX : taille : $330 \times 65 \mu$. A l'état de contraction, beaucoup plus larges que longs, sinon presque carrés $(200 \times 130 \mu)$ (fig. 35 et 36 ).

\section{Appareil reproducteur.}

\section{A) Système génital mâle.}

Testicules : les trois testicules, étant donné la contracture du strobile, sont situés en ligne en avant des glandes femelles ; ils emplissent la presque totalité du parenchyme 
médullaire des anneaux mâles. Taille : $7,0 \mu \times 120 \mu$ d'épaisseur. Leur position naturelle sans l'entrave due à la contraction est du type C. Ils ont une nette tendance à l'involution dans les derniers anneaux femelles mûrs.

VÉsicule sÉminale externe: ovalaire, située dans le prolongement de la poche du cirre ou bien dorsalement au-dessus de sa portion antiporale. 100-180 $\times$ 50-60 $\mu$.

POCHE DU CIRRE: fortement développée dans les anneaux mûrs. Mesure 130-170 $\times$ 40-45 $\mu$ de diamètre dans des anneaux de $370 \mu$ de large, atteignant et parfois dépassant les C.O.R. antiporaux $(\mathrm{PC} / \mathrm{AM}=1 / 2$ à $1 / 1,5)$. Parois fortement musculeuses formées de fibres longitudinales. Présence de quelques fibres rétractrices antiporales. La vésicule séminale interne située dans les $2 / 3$ postérieurs de la poche se poursuit par un canal déférent décrivant une large boucle avant d'aborder le cirre.

CIRre : cylindro-conique, glabre, long à l'état d'évagination de 50-55 $\mu \times 7,5 \mu$ de diamètre basal et $2 \mu$ de diamètre terminal. Se termine par un stylet filiforme de $55 \mu$ de long dans les exemplaires examinés.

\section{B) Système génital femelle.}

OvaIre : faiblement bi- ou tri-lobé, allongé transversalement en arrière des testicules. Mesure jusqu'à $200 \mu$ de large $\times 20 \mu$ de hauteur (dans un anneau de $330 \mu \times 40 \mu)$.

Vitellogène : ovoïde, postérieur à l'ovaire : $60 \times 25 \mu$ et dorsal par rapport à cette glande.

RÉceptacle séminal: ovoïde, situé en avant du lobe poral de l'ovaire ; $90 \times$ 35-40 $\mu$; ne dépasse pas les C.O.R. poraux.

VAGIN : non visible sur les préparations.

ANNEAUX GRAVides : utérus sacculaire dépassant latéralement les C.O.R. et contenant un grand nombre d'œufs. Bien qu'observés très immatures, ceux-ci présentent une forme allongée qui doit vraisemblablement persister à maturité en s'accentuant. Encore dépourvus de crochets, ils mesurent $60 \times 18 \mu$ (fig. 37).

\section{Diseussion.}

Les Echinocotyle d'origine française et congolaise ne se distinguent pas les uns des autres.

En se basant sur les crochets du rostre dessinés par Krabbe en 1869 et sur la taille des strobiles - seuls caractères des types qui soient connus avec exactitude on peut estimer que l'espèce de Rudolphi a bien été retrouvée ; aucune autre ne pourrait d'ailleurs la remplacer, bien qu'avec $H$. (E.) dubininae (Clerc, 1902) Deblock et Rosé, 1962 et H. (E.) magnisaccis (Meggitt, 1927), elle forme un groupe morphologiquement très homogène ; seule la taille des crochets $(35,87,70 \mu$ respectivement) réussit à en individualiser les espèces. D'autre part, relevant du sous-genre Echinocotyle, H. longirostris ne se distingue plus de E. multiglandularis Baczynska, 1914, dont nous 
avons étudié les exemplaires de Meggitt, vraisemblablement co-types de ceux de Baczynska, en provenance des collections de Looss d'Egypte.

Deux espèces d'Hymenolepis (nitidulans sensu nov. et lauriei) ont déjà été trouvées parasites aussi bien des Lari que des Charadrii; cette double localisation possible ne paraît donc pas très exceptionnelle de la part de ces petits Cestodes. E. druzniensis Jarecka, 1958, parasite de Larus en Pologne, à crochets falciformes de $42 \mu$ (Korpaczewska ne mentionne nulle part la taille exacte des crochets dans sa description de l'adulte, en 1959) se distingue des trois Echinocotyle précités par son cirre épineux, caractère exceptionnel des Echinocotyle puisque on ne le retrouve que chez E. rosseteri parasite d'Ansériformes (in Czaplinski, 1956).

\section{1) HYMENOLEPIS (ECHINO.) MAGNISACCIS}

(Meggitt, 1927) nov. comb.

nec Hymenolepis (Hym.) m. Meggitt, 1927.

Syn.: Echinocotyle glareolae Singh, 1952.

Hym. (E.) sp. inq. $\mathrm{n}^{\circ} 3$ Deblock et Rosé, 1962.

Région paléarctique: Afrique (Egypte).

Région indienne: Indes.

Hôte : Erolia minuta (Leisl.), Tringa glareola L.

Matériel observé : $1^{\circ}$ types de Meggitt, (coll. de Looss, British Museum de Londres), constitués d'un scolex attenant à des anneaux mâles et de plusieurs fragments de strobiles assez déformés; certains sont difficilement lisibles. $2^{\circ}$ Matériel de Baer, 1959 en provenance d'un Erolia minuta (Leisl.) de Tabuya (Congo ex-Belge) mélangé à Hym. (Echino.) longirostris en plus rares exemplaires (4).

\section{Description de l'espèce-type (planche 10, fig. 38-40)}

Strobile : $1,7 \times 0,22 \mathrm{~mm}$ de largeur maximale au niveau des anneaux mûrs. Le Cestode mûr convenablement étendu mesure sans doute 2,5 à $3,5 \mathrm{~mm}$; il doit être constitué de moins d'une centaine d'anneaux : une trentaine de cou, 10 à 15 anneaux mâles, autant de femelles et autant de gravides.

Anneaux mâles et femelles: $145 \times 80 \mu$. Anneaux gravides : $220 \times 160 \mu$.

Atrium GÉnITAL : garni d'une couronne de petits crochets de $5 \mu$ de long. Présence d'un sac accessoire de $35 \times 19 \mu$ de diamètre à lumière armée d'épines, et entouré de cellules glandulaires.

Musculature longitudinale interne formée vraisemblablement de 4 faisceaux par face.

(4) De ce fait, seuls les crochets $(b)$ de la figure 60 (in Baer, 1959, p. 98) correspondent à $H$. (E.) longirostris; les scolex $(a)$ sont ceux de l'espèce $H$. (E.) magnisaccis. 
Scolex : typique du sous-genre Echinocotyle. $130 \mu$ de long $\times 170 \mu$ de diamètre. Ventouses en ovale très allongé $106 \times 65 \mu$ de large; leur bord musculeux de 10-13 $\mu$ est garni de 20 à 25 groupes de 3-4 petits crochets de 7,5 $\mu$ de long disposés en éventail. Le fond des ventouses, nettement aplati, est garni de 4-5 rangées longitudinales des mêmes épines (fig. 38).

SAC DU ROSTRE : $120 \times 50 \mu$ dépassant peu le niveau du bord postérieur des ventouses.

Rostre: non observé évaginé, garni d'une couronne de 10 crochets falciformes de $74 \mu$ (fig. 39).

Cou : court de $100 \mu$ de large.

\section{Appareil Reproducteur.}

\section{A) Appareil mâle.}

Protérandrie nette; trois TESTICUles de $45 \mu$ environ de diamètre à maturité, disposés selon le type $\mathrm{C}$ ou $\mathrm{D}$, principalement dans les anneaux mâle jeunes.

VÉsicule séminale externe disposée au niveau de l'axe médian du strobile, ovoïde ; $35 \times 50 \mu$ mesurant jusqu'à $115 \times 25 \mu$ dans les anneaux gravides.

POCHE DU CIRRE : en fuseau étroit allongé, très musculeux ; mesurant de 80-95 $\mu \times$ 27-35 $\mu$ dans les anneaux mâles, s'allongeant jusqu'à $140 \times 32 \mu$ dans les anneaux gravides. Elle dépasse les C.O.R. antiporaux. PC/ $\mathrm{AM}=1 / 1,4$ environ.

CIRre : cylindro-conique, glabre, mesurant 40-45 $\mu$ à l'état d'évagination partielle ; $7,5 \mu$ de diamètre à la base. Non observé totalement évaginé. Les organes de la poche du cirre ne sont pas observables (fig. 40).

\section{B) Appareil femelle.}

Assez mal visible. Ovaire probablement bilobé, mesurant $60 \times 25-30 \mu$. VitelloGÈNE postérieur à l'ovaire, $25 \times 15 \mu$. RÉCEPTACLE SÉmINAL ovoïde, médian, $50-55 \times$ $30 \mu$. VAGIN non perceptible. Anneaux gravides: UTÉrus sacculaire non lobé dépassant de part et d'autre les C.O.R. longitudinaux. Eufs (non mûrs) : ovoïdes, de $19 \times 15 \mu$.

\section{Discussion.}

Ne pensant pas que les types de $H$. magnisaccis Meggitt puissent exister encore, nous avions pris, en 1962, la décision de supprimer cette espèce. Ces types retrouvés montrent qu'elle appartient au sous-genre Echinocotyle; celle de Baer en provenance du Congo ne s'en distingue pas morphologiquement sauf par de petites différences de mensurations non significatives (strobile $3 \mathrm{~mm}$; sac accessoire : $56 \times 28 \mu$ de diamètre ; poche du cirre : $125-150 \times 20 \mu$ de diamètre ; cirre de $80 \mu \times 9 \mu$ de diamètre basal ; testicules de 45 à $60 \mu$ de diamètre ; ovaire mûr : $113 \times 45 \mu$. Anneau mûr : 250$280 \times 90 \mu$ de haut) ; néanmoins les crochets du rostre ont exactement la même taille $(74 \mu)$ et le même rapport lame/manche ; ces caractères nous paraissent suffisants pour assurer la similitude des deux lots de Cestodes africains, de même que celle d' $H$. gla- 
reolae Singh, 1952 et de Hymenolepis (Echino.) sp. inq. n” 3 Deblock et Rosé, 1962 dont les crochets sont également identiques.

A notre avis, la différence de longueur de 15 à $20 \mu$ gui sépare les crochets de $H$. magnisaccis de ceux de $H$. dubininae (Olerc, 1902) (6) est suffisamment significative pour séparer les deux espèces. Des récoltes de l'une et de l'autre plusieurs fois répétées, bien séparées géographiquement et chronologiquement, ont montré la fixité de cette différence ; elle correspond probablement à deux espèces distinctes.

\section{2) HYMENOLEPIS (HYM.) NITIDULANS (Krabbe, 1882)}

Syn.: Hym. charadrii Yamaguti, 1935.

Hym. nitidulans (Krabbe) sensu Southwell, 1924.

Matériel observé : Spécimens de Southwell, 1924 (Grande-Bretagne).

Depuis 1924, cette espèce n'était très imparfaitement connue que par la description erronée de Southwell (5); l'examen des préparations de cet auteur donne raison à l'ensemble des hypothèses que nous formulions en 1962 (p. 804-805) : il s'agit bien de $H$. $(H$.$) charadrii Yamag., 1935, tel que nous l'avions redécrit en 1960$ et en 1962.

Les types de $H$. nitidulans (Krabbe, 1882) paraissent avoir été perdus ; dans l'état actuel de nos connaissances, il semble bien qu'aucun Hymenolepis de Charadriiforme autre que $H$. charadrii ne puisse posséder des crochets falciformes de 50-60 $\mu$. En conséquence, nous faisons tomber les deux espèces en synonymie; le Cestode décrit par Southwell ne change donc pas de nom.

\section{3) HYMENOLEPIS (HYM.) PORALE Meggitt, 1927}

Matériel examiné: Types de Meggitt (British Museum de Londres) constitués de trois préparations contenant les fragments non successifs du même strobile.

\section{Description de l'espèce-type (planche 11, fig. 41 à 45)}

STrobile : $25 \times 2 \mathrm{~mm}$ d'après Meggitt. Vraisemblablement plus long (40 à $60 \mathrm{~mm})$. Naturellement assez aplati.

PoREs GÉNITAUX : unilatéraux situés au milieu du bord de l'anneau.

Atrium génital : long de $40 \mu$ à lumière étroite. Présente un sac accessoire inerme complexe, subdivisé en quatre digitations identiques. Diamétralement opposées deux à deux, elles divergent à partir du pertuis atrial pour chapeauter l'extrémité porale de la poche du cirre. Chacune des quatre digitations mesure $20 \mu$ de longueur $\times 10-13 \mu$ de diamètre, la paroi, hyaline, étant épaisse de 4-5 $\mu$. L'embryogénèse montre que la formation prend naissance non aux dépens de la paroi de l'atrium génital, mais du massif cellulaire situé à l'emplacement du futur pore génital, avant son percement (fig. 42, A, B, C).

(5) La supposition de la note infrapaginale 11 (1962, p. 804) s'est révélée exacte.

(6) nom. nov. pour Echinocotyle nitida sensu Clerc, 1902-1903 nec Krabbe, 1869. Voir Deblock et Rosé, 1962, p. 802-804. 
Le parenchyme cortical antérieur au pore génital est garni d'un amas cellulaire bien différencié qu'on ne retrouve pas du côté postérieur ou dans le parenchyme cortical antiporal.

Musculature longitudinale externe formée de 70 à 80 bandes fines de $2,5 \mu$ de diamètre ; l'interne est formée de 25 à 30 bandes de 7-13 $\mu$ de diamètre.

C.O.R. : formés de deux canaux fins superposés; les C.O.R. ventraux sont reliés par une anastomose transverse à la partie postérieure de chaque anneau.

ScOLEX : inconnu.

Cou : étroit ; $100 \mu$ de diamètre.

AnNEAuX: beaucoup plus larges que hauts et plats. Taille : 1.200 à $1.350 \mu \times$ $180 \mu$.

\section{Appareil reproducteur.}

Comme l'avait figuré Meggitt (pl. XXX, fig. 46), le massif des glandes reproductrices est décalé légèrement du côté poral (fig. 41).

\section{A) Anneaux mâles.}

Testicules : disposés en triangle à pointe postérieure (type D), très serrés les uns à côté des autres; le médian semble être le plus dorsal. Plus ou moins lobés. Petits : $50 \mu$ de diamètre.

POCHE DU CIRRE : fusiforme, allongée, $340 \times 60 \mu$ de diamètre maximal ; n'atteint pas le niveau du testicule poral ni l'axe longitudinal du strobile. $\mathrm{PC} / \mathrm{AM}=1 / 3,5$. Parois minces $(3 \mu)$. Contient un cirre puissant.

Cirre invaginé : cylindro-conique allongé $(80 \mu)$, aux parois épaissies fortement colorables, finement spinulées : les épines sont très serrées, formant comme un velours, fines et courtes $(2 \mu)$, toutes égales entre elles.

Le cirre, très partiellement évaginé, apparaît très puissant, armé et épais $(30 \mu$ de diamètre à la base).

Le canal déférent intervésiculaire est long et fin; la vésICULE SÉMINALE EXTERNE, vide de spermatozoïde, est petite et située en avant des glandes sexuelles.

\section{B) Anneaux femelles.}

Sur les types, l'appareil femelle n'est observable que très immature, à l'exception du vagin.

OvaIRE : petit, comme les testicules, $50 \mu$ de diamètre ; ventral, polylobé, situé au milieu du groupe des testicules.

VITELlogène : invisible.

VAGIN : ventral, long de $420 \mu$ environ. Portion distale tubulaire de parois minces, à lumière large. $130-140 \mu \times 15 \mu$ de diamètre. Portion proximale longue à lumière plus étroite. Absence de sphincter et de fibres rétractrices.

RécePtACle séminal: non développé, ventral, en dessous du testicule poral. Ovoïde : $20 \times 30 \mu$. 
ANNEAU GRAVIDE : subit un élargissement considérable : 1.800-1.900 $\mu$ de large $\times 250 \mu$ de haut.

UTÉRUs: emplissant le segment et s'étendant latéralement au-delà des C.O.R. Polylobé (fig. 44).

CEuFs MûRs : allongé, en fuseau à bouts arrondis. Embryon hexacanthe : 25-30 $\times 12 \mu$ de diamètre. Crochets de $9 \mu \times 2,5 \mu$ de haut. Coque externe mince : 90-100 $\times$ 20-25 $\mu$ de diamètre (fig. 45).

\section{Discussion.}

Le Cestode de Meggitt, en dépit de l'absence de son scolex, s'individualise facilement par la possession: $1^{\circ}$ d'un cirre finement épineux très volumineux; $2^{\circ}$ d'un sac accessoire de conformation inédite par sa subdivision en quatre poches symétriques. Ce dernier s'apparente par son importance et sa situation aux formations analogues, mais seulement bisacculées, de l'Hymenolepis bisaccata Fuhrm., 1906, parasite d'Ansériforme et de l'Hymenolepis (= Biglandatrium) biglandatrium (Spasskaïa, 1961), parasite de Colymbiforme (in Spassky, 1963, p. 227).

H. porale sensu Dubinina, 1953 dépourvu de sac accessoire n'est sûrement pas identifiable à l'espèce redécrite.

14) HYMENOLEPIS (HYM.) RECURVIROSTRAE (Krabbe, 1869)

Syn. : H. himantopodis (Krabbe, 1869) sensu Meggitt, 1927.

H. recurvirostrae (Krabbe, 1869) sensu Meggitt, 1927.

H. recurvirostrae (Krabbe, 1869) sensu Voge et Read, 1954.

nec $H$. recurvirostrae magnosacco Joyeux, Baer et Martin, 1936.

H. recurvirostrae sensu Joyeux et Baer, 1940.

H. recurvirostrae sensu Baer, 1959.

H. recurvirostrae sensu Dubinina, 1953.

Région paléarctique : Afrique (Egypte: Krabbe et Meggitt).

Région néarctique: (U.S.A., Californie : Voge et Read).

Hôtes : Recurvirostra avocetta L., Recurvirostra americana.

Matériel observé : $1^{\circ}$ Types de Krabbe (coll. de Bilharz, Egypte). $2^{\circ}$ Exemplaires de H. recurvirostrae (coll. Looss d'Egypte). $3^{\circ}$ Exemplaires anonymes de provenances diverses (Musée de Vienne) et inédites. $4^{\circ}$ Exemplaires de Meggitt correspondant à $H$. himantopodis sensu Meggitt et à $H$. recurvirostrae (Krabbe) (British Museum, Londres). $5^{\circ}$ Exemplaires de Voge et Read (dus à l'amabilité de Mme M. Voge). $6^{\circ}$ Exemplaires de Dubinina.

Description de l'espèce-type (planche 12, fig. 46 à 52).

Strobile : le Cestode complet, si l'on en juge par ses fragments, doit être long de 50 à $60 \mathrm{~mm}$ au moins, assez plat naturellement et large jusqu'à $1,5 \mathrm{~mm}$; il doit comporter un grand nombre d'anneaux (1.000 au moins).

PORES GÉNITAUX: unilatéraux dextres. 
Atrium génItAL : court, tubulaire (10 à $20 \mu$ de long), dépourvu de sacculus et d'épine.

Musculature longitudinale : 25 à 30 B.M.I. par face.

C.O.R. : dépourvus d'anastomose ventrale transverse.

SCOLEX : assez volumineux. Lorsque les 4 ventouses s'ouvrent en avant, son sommet s'aplatit et il affecte la forme d'une pyramide à base carrée. 180 à $300 \mu$ de hauteur $\times 260$ à $300 \mu$ de diamètre maximal (fig. 46).

Ventouses: sphériques 100 à $160 \mu$ de diamètre. Bord musculeux inerme de 10 à $18 \mu$ de large.

SAC DU ROSTRE : $150-200 \times 90-100 \mu$ de diamètre. Son fond dépasse le niveau du bord postérieur des ventouses.

Rostre ÉVAginé : 190 à $230 \mu$ de long $\times 80-90 \mu$ de diamètre. La table apicale est relativement volumineuse, presque toujours globuleuse, de 70 à $90 \mu$ de diamètre. Les petits crochets qu'elle porte sont de ce fait largement espacés les uns des autres. 10 crochets chéliformes disposés en une couronne unique msurant 14-15 $\mu \times 8 \mu$ de hauteur sur les types, et certains exemplaires de Looss et du musée de Vienne. Néanmoins, il en existe de plus petits : 9-10 ×6

Cou : souvent étroit $(100-150 \mu)$ et long : $700 \mu$.

ANNEAUX : beaucoup plus larges que hauts, plats.

Anneaux mâles : $460 \times 50 \mu$, mûrs : $510 \times 70 \mu$, gravides jeunes : $1.500 \times 115 \mu$, mûrs : $1.250 \times 140 \mu$.

\section{Appareil reproducteur.}

\section{A) Système mâle.}

Protérandrie accusée. Testicules non persistants dans les anneaux femelles mûrs. Testicules relativement peu visibles dans les anneaux mâles. 30-38 $\mu$ de diamètre. Disposés selon le type G (en ligne) (fig. 49).

VÉsicule séminale externe: ovoïde, ou sphérique. Située dorsalement à l'extrémité de la poche du cirre ou dans son prolongement. 90-100 $\times 55 \mu$ de diamètre.

Poche DU CIRRE : de forme allongée, dépassant souvent largement les C.O.R. poraux pour atteindre le milieu de l'anneau, sans le dépasser, sauf dans certains anneaux mâles. Parois musculeuses minces $(2 \mu)$. Formée de fibres longitudinales non hélicoïdales. Elle mesure, suivant la maturation de l'anneau, de 180 à $270 \mu$ de long $\times$ 30-35 $\mu$ de diamètre. $\mathrm{PC} / \mathrm{AM}=1 / 3$.

CIRRE invaginé difficilement observable ne laissant aucunement présager de son aspect évaginé ; une légère dilatation terminale permet l'observation de fines épines. Cirre évaginé régulièrement cylindrique sur toute sa longueur, de $100 \times 6 \mu$; sa base est finement spinulée (épines punctiformes de $1 \mu$ ); sa partie distale présente des épines plus fortes et aplaties, en forme d'épine de rosier (fig. 48).

\section{B) Système femelle.}

Dans les anneaux mâles, seuls se différencient nettement le vagin et le réceptacle 
séminal. Le massif embryonnaire des glandes femelles se situe entre les deux testicules poraux.

OvaIRE: allongé transversalement, sensiblement au milieu de l'anneau, pourvu d'une faible bilobation. Mesure $160-180 \mu$ de large $\times 55 \mu$ de haut.

VITEllogène: ventral par rapport à l'ovaire et partiellement sous lui. Ovoïde ; $35 \times 45 \mu$.

RÉCEPTACLE SÉminal: situé sous l'aile porale de l'ovaire. Ovoïde, ne dépassant pas les C.O.R. poraux ; 70-90 × 45-55 $\mu$.

VAGIN proximal, membraneux et tubulaire de faible section; distal, tubulaire ne dépassant pas les C.O.R. poraux ; 40-60 × 7-10 $\mu$ de diamètre. Parois légèrement chitinisées, lisses et minces (1-2 $\mu$ ) lorsque la lumière est dilatée ; de forme cylindro-conique à parois plissées sur les exemplaires de Voge et Read, le sommet occlus de fibres annulaires peu visibles (fig. 52). Absence de sphincter et de fibres rétractrices.

UTÉRUS : inapparent dans les anneaux femelles mûrs. Dans les anneaux gravides : allongé transversalement, dépassant les C.O.R. de part et d'autre; sacculaire à bords festonnés. L'anneau gravide contient proportionnellement une poche de cirre beaucoup plus courte que dans la partie antérieure du strobile, par suite de l'élargissement de l'anneau: $230-270 \times 35 \mu$ (dans un anneau de $1.500 \times 115 \mu)$. Utérus contenant de nombreux œufs (fig. 51).

CEufs mûRs : sphériques ou légèrement ovoïdes. 39-41 × 32-37 $\mu$. Coque interne légèrement et régulièrement épaissie $(1,5-2,5 \mu)$ (fig. 48).

Remarque. - Certains exemplaires se différencient de l'espèce-type par des mensurations différentes et notamment des strobiles aussi longs, mais beaucoup plus étroits, l'anneau gravide ne dépassant pas $365 \mu \times 55 \mu$ avec une poche du cirre de $110 \times 18 \mu$; il ne contient qu'un petit nombre d'œufs de $37 \times 28 \mu$ disposés en deux rangées superposées. PC/AM $=$ 1/3. L'ovaire parfois réniforme à concavité postérieure, peut être fortement multilobé (5-6 lobes).

La vésicule séminale externe peut se replier dorsalement à la poche du cirre, au-dessus de la partie moyenne de celle-ci et du réceptacle séminal.

D'autre part, le vagin distal peut avoir, soit l'aspect cylindro-conique à parois plissées que nous avons figuré, soit après déplissement, un aspect simplement tubulaire; il est alors plus dilaté sur tout son parcours.

\section{Discussion.}

Il devient évident que, depuis sa description par Krabbe, $H$. recurvirostrae n'est cité à bon escient dans la littérature que par deux auteurs :

$1^{\circ}$ Dans la même région géographique, par Meggitt; mais cet auteur commit l'erreur de mentionner la même espèce sous le nom différent de $H$. himantopodis, sans d'ailleurs exposer les motifs de ses conceptions de discrimination.

$2^{\circ}$ D'une façon plus inattendue, étant donné l'éloignement géographique de la Californie et de la localisation-type, par Voge et Read. 
H. recurvirostrae magnosacco Joyeux et coll., 1936 (Somalie) est en réalité une espèce différente (cf. plus loin Hym. $(H$.$) tsengi) ; H. recurvirostrae sensu Joyeux et$ Baer, 1940 (Madagascar) que nous suggérions être individualisable est bien un Hymenolépididé, mais ne possède qu'un seul testicule; il relève par conséquent du genre Aploparaksis. H. recurvirostrae sensu Baer, 1959 (Congo) est lui aussi identifiable à Hym. $(H$.$) tsengi (le dessin de la fig. 64, p. 104$ correspond à l'anatomie génitale d’un anneau encore immature dépourvu de glandes sexuelles), de même que $H$. recurvirostrae sensu Dubinina 1953.

Quant aux spécimens de l'Oural (Clerc, 1910 dont la publication russe ne nous est pas accessible) on ne peut présumer de leur identité exacte.

Les présentes redescriptions des cinq espèces proposées par Baer en 1959, p. 103 pour tomber en synonymie avec $H$. recurvirostrae (Krabbe) montrent que, en dehors de la similitude de leur crochet, elles sont anatomiquement toutes différentes les unes des autres.

$$
\text { 栾 }
$$

\section{5) HYMENOLEPIS (HYM.) RECURVIROSTROIDES Meggitt, 1927}

Syn.: Hymenolepis recurvirostrae sensu Joyeux et Baer, 1940.

Matériel observé: Types de Meggitt (British Museum) et de Joyeux et Baer.

Ces deux espèces, bien qu'Hyménolépididés typiques, ne possèdent qu'un seul testicule ; elles doivent être placées dans le genre Aploparaksis; elles paraissent semblables l'une et lautre. formes.

H. recurvirostroides est à rayer définitivement de la liste des Hymenolepis de Charadrii-

$$
\text { 논 }
$$

16) HYMENOLEPIS (HYM.) TSENGI Joyeux et Baer, 1940

Syn. : Hym. himantopodis sensu Shen Tseng, 1933 nec Krabbe, 1869.

Hym. recurvirostrae var. magnosacco, Joyeux, Baer et Martin, 1936.

Hym. himantopodis sensu Baer, 1940 nec Krabbe.

Hym. recurvirostrae sensu Dubinina, 1953 nec Krabbe.

Hym. recurvirostrae sensu Baer, 1959 nec Krabbe, pro parte (6).

Hym. edouardensis (Baer, 1959) Deblock et Rosé, 1962 (p. 810).

Hym. himantopodis sensu Singh, 1959 nec Krabbe.

Hym. himantopodis (Krabbe, 1869) sensu Joyeux (inédit) (7).

Hym. himantopodis (Krabbe, 1869): exemplaires de collections diverses (Musées de

Berlin et de Vienne). Inédit.

nec H. recurvirostrae sensu Baer, 1959 (p. 103), pro parte.

Localisation géographique :

Région paléarctique: Afrique (Somalie, Congo ex-Belge); Asie (Sibérie occidentale, Chine) ; Europe (France méridionale) (7).

(6) Spécimens de Mwiga, Lac Edouard (Congo ex-belge).

(7) Observation inédite de Joyeux, 1952. Etang du Canet (P.-O.). 
Région indienne : Indes.

Région néotropicale: Antilles (Antigua).

Hôte : Tringa totanus (L.): Shen Tseng (Chine).

Himantopus mexicanus Mull. : Baer (Antilles).

Himantopus himantopus (L.): Baer (Congo Belge); Joyeux (France); Singh (Indes). Recurvirostra avocetta L.: Dubinina (Sibérie occidentale).

Matériel observé : $1^{\circ}$ Types de Joyeux et Baer, 1940. $2^{\circ}$ Spécimens de Shen Tseng, 1933. $3^{\circ}$ et $4^{\circ}$ de Baer, 1940 et $1959.5^{\circ}$ Spécimens inédits de Joyeux (Le Canet, 1962). $6^{\circ}$ et $7^{\circ}$ Spécimens d'Himantopus himantopus des Musées de Berlin et de Vienne, étiquetés sans autre indication, dont plusieurs bonnes coupes histologiques. $8^{\circ}$ Spécimens aimablement communiqués par Mme M. N. Dubinina.

N.B. - La description est faite d'après les préparations de Shen Tseng représentant les premiers spécimens de l'espèce récoltés (coll. Faust). Nous n'avons établi la synonymie complète de l'espèce qu'ultérieurement; les similitudes étant manifestes nous n'avons pas cru indispensable de recommencer l'ensemble de la description uniquement d'après les types.

Description de l'espèce (planche 13, fig. 53 à 60).

Strobile : de longue taille: $60-70 \mathrm{~mm} \times 1,5$ de largeur maximale. Formé d'un très grand nombre d'anneaux (1.000-1.500) beaucoup plus larges que hauts.

PORES GÉNITAUX : unilatéraux dextres.

Atrium génital: caractéristique de l'espèce. Dépourvu de sacculus et d'épines. Nettement plus développé que dans la plupart des autres espèces d'Hyménolepis de Charadriiforme. Son fond est disposé en forme de cuvette ou de cratère de 30-40 $\mu$ de diamètre sur 20-30 $\mu$ de profondeur centrée sur l'orifice mâle ; sa paroi épaissie $(5 \mu)$ est tapissée d'un grand nombre de verrucosités coniques de $2,5 \mu$ de diamètre environ et $2,5 \mu$ de hauteur, disposées de façon concentrique, bien visibles dans les portions jeunes du strobile, mais persistantes dans les proglottis mûrs. Cet atrium est souvent entouré d'un anneau plus colorable dû à une nette condensation cellulaire périphérique (fig. 58, 59).

Musculature longitudinale externe: 40 à 50 faisceaux; interne formée fondamentalement de 4 faisceaux de section volumineuse; mais l'échange assez fréquent de fibres avec les faisceaux voisins augmente légèrement ce nombre sur les coupes transversales (fig. 60).

C.O.R. : dépourvus d'anastomose transverse entre les canaux ventraux, bien qu'il existe des amorces, plus ou moins longues, de collatérales. Partagent le strobile en trois zones longitudinales égales. Ventraux par rapport aux conduits génitaux.

SCOLEX: de forme massuée, sans démarcation nette avec le cou, apparentée à celle de $H$. (H.) hughesi par exemple, mesurant $220 \mu$ de long environ $\times 275 \mu$ de diamètre. Ventouses sphériques de $125 \mu$ de diamètre, inermes. Sac du rostre court, ne dépassant pas en arrière le bord postérieur des ventouses, $150 \mu$ de long $\times 90 \mu$ de diamètre. Rostre court non observé évaginé (fig. 53).

Crochets: chéliformes, disposés en une couronne de 10, de petite taille: 
8,7-9,5 $\times$ 4,5 $\mu$ de hauteur (8). La garde n'est pas spécialement spatulée; elle est sensiblement de même longueur que la lame, tantôt légèrement plus courte, tantôt plus grande (fig. 54).

Cou : $230 \mu$ de diamètre environ. La strobilisation s'amorce 400-500 $\mu$ en arrière du scolex.

ANNEAUX: beaucoup plus larges que hauts.

\section{Appareil reproducteur.}

\section{A) Système mâle.}

Testicules: protérandrie accusée. Testicules non persistants dans les anneaux femelles mûrs ou en voie de maturation; à disposition prédominante en ligne (type $\mathrm{G}$ ). 55-70 $\mu$ de diamètre (fig. 56).

VÉsicule sÉminale eXterne: dorsale, située dans le prolongement de la poche du cirre. Ovoïde, $180-220 \times 44-55 \mu$ de diamètre.

POCHE DU CIRRE : bien développée ; taille variable en fonction de l'âge de l'anneau ; proportionnellement beaucoup plus longue dans les anneaux mâles où elle atteint facilement l'axe longitudinal du strobile, le dépassant légèrement même: PC/Anneau mâle $=1 / 3$. Dans les anneaux femelles mûrs, son fond se situe seulement dans le voisinage immédiat des C.O.R. poraux : $\mathrm{FC} / \mathrm{AM}=1 / 3,5$ à $1 / 4$. Taille : $360-400 \times$ 55-65 $\mu$ de diamètre, parfois jusqu'à $480 \mu$ (dans les anneaux de $1.500 \mu$ de large). Parois fortement musculeuses, épaisses de 7-11 $\mu$ formées de fibres longitudinales non hélicoïdales. De puissantes fibres rétractrices s'insèrent depuis son fond jusqu'au côté antiporal de l'anneau.

VÉsicule SÉminale INTERNE: située dans les $2 / 3$ postérieurs de la poche se poursuivant du côté poral par un canal éjaculateur décrivant une courte boucle avant d'atteindre le cirre.

CIRre Évaginé : régulièrement cylindrique ; 50 à $90 \mu$ de long $\times 10 \mu$ de diamètre à la base ; il peut être considéré comme glabre ; néanmoins, sur une longueur d'une quarantaine de microns, on peut observer une spinulation punctiforme dans la lumière des cirres invaginés, plus particulièrement visible sur les coupes histologiques.

\section{B) Système femelle.}

Ne sont mûrs dans les anneaux mâles que le réceptacle séminal et le vagin.

OVAIRE : postérieur, ventral et médian. Trilobation majeure compliquée sur certains exemplaires d'échancrures plus accusées ; taille : 340-365 $\times 70-90 \mu$ de haut dans les anneaux contractés (fig. 57).

VITELlogène: médian, postéro-ventral par rapport à l'ovaire. Souvent de 3 à 6 lobes. Taille : $100-120 \times 44-60 \mu$.

RÉCEPTACle SÉminal: situé en avant du lobe poral de l'ovaire, ne franchissant pas les C.O.R. poraux : Ovoïde : $160-180 \times 45-70 \mu$ de diamètre.

VAGIN DISTAL : caractéristique de l'espèce. Cylindro-conique à parois légèrement

(8) Des exemplaires en provenance du Musée de Berlin présentent des crochets de $5-5,5 \mathrm{u}$ de long seulement. 
chitinoïdes. Son extrémité antiporale est occluse par un très volumineux sphincter de $30 \mu$ de diamètre au niveau duquel s'insère un très puissant muscle rétracteur dont les fibres viennent rapidement se confondre avec leurs homologues de la poche du cirre. De ce fait, le vagin distal est plus ou moins étiré : le sphincter, suivant les strobiles ou les anneaux d'un même strobile, peut se disposer de part ou d'autre des C.O.R. poraux, souvent jusqu'au milieu de l'anneau dépassant même le fond de la poche du cirre ; court, le cône qu'il figure est très élargi; étiré, il s'amincit. Taille courte : $170 \times 55-60 \mu$ de diamètre externe. Taille longue : $330 \mu \times 30 \mu$ de diamètre externe (dans des anneaux de $780 \mu$ de large) et jusqu'à $480 \mu$ dans des anneaux gravides de $1.500 \mu$. Sa structure complexe est représentée à la figure 59, pl. 13. Sa lumière est tapissée de papilles; il est entouré d'un volumineux manchon de cellules glandulaires qui se laissent colorer d'une façon plus ou moins intense. Le vagin proximal, membraneux, n'a pas été observé.

UTÉRUS: sacciforme à bords festonnés, dépassant latéralement les C.O.R.

Eufs: (d'après exemplaire de Joyeux; immatures, les crochets n'étant pas définitivement formés). Coque externe, régulièrement sphérique, mince, de 38 à $46 \mu$ de diamètre. Coque interne plus ovoïde, légèrement épaissie d'une façon uniforme de $30 \times 20 \mu$ (fig. 55).

\section{Discussion.}

L'atrium génital verruqueux joint à l'anatomie des conduits génitaux caractéristiques de cet Hymenolepis facilite une identification dépourvue d'ambiguïté qui la distingue notamment de $H$. himantopodis Krabbe (cf. la discussion de cette dernière espèce p. 703) ; elle entraîne les synonymies proposées. Nous y rattachons l'espèce de Singh, 1959 exactement conforme aux types.

\section{*}

17) HYMENOLEPIS (HYM.) VAGINATA Baczynska, 1914

Syn. : Hymenolepis innominata Meggitt, 1927.

Matériel observé : Types de Baczinska. Types de Meggitt (British Museum de Londres). Il est manifeste que les préparations des deux auteurs résultent du partage d'un unique lot initial, provenant des collections égyptiennes de Looss.

Description de l'espèce-type (planche 14, fig. 61 à 63).

Strobile: vraisemblablement de plus grande taille que les $40 \mathrm{~mm}$ de Baczynska, les $10 \mathrm{~mm}$ de Meggitt étant une erreur manifeste $(10 \mathrm{~cm}$ ?). Largeur maximale : $1.150 \mu$ au niveau des anneaux mâles seuls observés.

PORES GÉNITAuX : unilatéraux dextres.

Atrium génItal: profond de $100 \mu$ environ dépourvu de sacculus; sa portion mâle est tapissée d'un velours de très fines papilles longues de 2 à $3 \mu$, en forme 
d'aiguilles, sans rapport morphologique avec la spinulation atriale des espèces du sousgenre Echinocotyle (fig. 63 et 63 A).

Musculature: longitudinale interne formée de 25 à 30 faisceaux par face.

C.O.R. : absence d'anastomose transverse; ventraux par rapport aux conduits génitaux.

Scolex : pyramidal, pourvu de 4 ventouses arrondies non spinulées et d'un rostre armé rétractile. Taille : 150-230 $\mu$ de haut $\times 200 \mu$ de diamètre. Ventouses : 80 à $100 \mu$ de diamètre. Sac du rostre: $130-170 \times 55 \mu$ de diamètre se terminant sensiblement au niveau du bord postérieur des ventouses. Rostre évaginé long de 140-200 × 45-55 $\mu$ de diamètre et de $300 \mu$ jusqu'au fond du sac. Crochets disposés en une couronne de 8 , mesurant suivant les scolex, de $35 \times 8$ à $42-44 \times 10 \mu$, claviformes, la garde étant noyée dans le prolongement du manche (fig. 61).

ANNEAUX: plus larges que hauts, trapézoïdaux. Le rapport de la longueur à la largeur $=1 / 4$ environ. Taille des anneaux mâles $1.150 \times 200-250 \mu$.

\section{Appareil reproducteur.}

\section{A) Système mâle.}

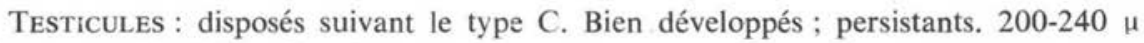
de large $\times 180-200 \mu$ (fig. 62).

Vésicule séminale eXterne : ovoïde, $210 \times 80 \mu$; située dorsalement en arrière de la poche du cirre. Le canal intervésiculaire qui la relie à la poche du cirre est long de $200 \mu \times 20 \mu$ de diamètre.

POCHE DU CIRRE : disposée obliquement par rapport au bord extérieur de l'anneau, et située de part et d'autre des C.O.R. poraux, son fond pouvant atteindre l'axe longitudinal du strobile. Taille maximale : $290 \times 100$ de diamètre. Parois minces $(4 \mu)$. Rapport $\mathrm{PC} / \mathrm{AM}=1 / 3$ à $1 / 4$. Absence de fibres rétractrices.

LA vÉSICUle SÉminAle INTERNE occupe, au niveau des $2 / 3$ postérieurs de la poche du cirre, un volume restreint par suite du grand développement du canal éjaculateur, très contourné, chitinoïde, qui parcourt deux fois la longueur de la poche à partir du sommet poral de la vésicule.

CirRe : observé seulement partiellement évaginé dans l'atrium génital, il est glabre et mesure $50 \times 25$ de diamètre à la base. Evaginé, il est sûrement beaucoup plus long $(300 \mu$ ?), et est peut-être terminé par un stylet.

\section{B) Système femelle.}

Les types ne contiennent pas d'anneau femelle mûr.

OVAIRE: situé ventralement entre les trois testicules. Vraisemblablement multilobé, les lobes devant être petits.

RÉCEPTACle sÉminal : ventral, ovoïde, ne dépassant pas les C.O.R. poraux, volumineux : $210 \times 130 \mu$ de diamètre; situé entre l'aile porale de l'ovaire et la poche du cirre. 
VAGIN PROXIMAL: tubulaire, membraneux, très sinueux, long de 200 à $250 \mu$. Absence de sphincter, mais présence de quelques fibres rétractrices nettes, s'insérant à la limite des deux parties vaginales.

VAGIN DISTAL : cylindro-cônique, chitinoïde, dépassant les C.O.R. poraux. 140-150 $\mu$ de long $\times$ 40-45 $\mu$ de diamètre externe et $13 \mu$ de diamètre interne. Sa lumière est tapissée de fines papilles. Il est entouré d'un manchon épais de cellules glandulaires. Sa structure s'apparente par conséquent à celle de $H$. $(H$.) mudderbugtenensis ou celle de $H$. (H.) tsengi (fig. 63 et $63 \mathrm{~B}$ ).

UTÉrus et EUfs inconnus.

\section{Discussion.}

Toutes les lames de Meggitt portent l'inscription « $H$. vaginata Baczynska secondairement barrées d'un trait. L'auteur ne devait donc pas ignorer l'existence de cette espèce. Il est dommage qu'il n'ait pas jugé utile de faire suivre la création de $H$. innominata d'une motivation de sa décision qui ne nous est pas clairement apparue.

19) HYMENOLEPIS sp. 805 Meggitt, 1927

Hôte : Erolia minuta (Leisl.).

Matériel observé : Spécimens de Meggitt (British Museum).

Cette espèce est un Aploparaksis.

*

\section{C) DESCRIPTION D'ESPECES NOUVELLES}

\section{1) HYMENOLEPIS (HYM.) APLOPARAKSIOIDIS n. sp.}

Syn.: Hymenolepis rectacantha sensu Baer, 1962.

Région paléarctique : Islande.

Hôte : Hoematopus ostralegus (L.).

Matériel observé : Deux scolex, nombreux strobiles contractés, coupes histologiques.

Description de l'espèce (planche 15, fig. 64 à 70).

STROBILE : filiforme ; complet et semi-contracté, mesure 15 à $20 \mathrm{~mm} \times 0,12$ à $0,21 \mathrm{~mm}$ de diamètre maximal ; les strobiles les plus longs doivent atteindre probablement jusqu'à $40 \mathrm{~mm}$. De section régulièrement circulaire. Comporte un très grand nombre d'anneaux (1.000-1.500) courts.

PoREs génItAuX : unilatéraux, dextres.

Atrium GÉnital : court et peu apparent. Dépourvu de sacculus et d'épines; dans certains anneaux mûrs et gravides, il s'extroverse et forme un bourrelet légèrement saillant à l'extérieur de l'anneau, de $15 \mu$ de diamètre environ. 
Musculature longitudinale: a) externe formée d'une quarantaine de fins faisceaux par face ; $b$ ) interne formée de quatre faisceaux relativement volumineux (5 à $7 \mu$ de diamètre) par face (fig. 70).

C.O.R. : les canaux ventraux sont souvent fort dilatés (15 à $30 \mu$ de diamètre) ; toujours dépourvus d'anastomose transverse. Ventraux par rapport aux conduits génitaux.

Scolex : petit : $130 \times 130 \mu$. Quatre ventouses musculeuses inermes de $80 \mu$ de diamètre. Un rostre puissant non observé évaginé. Sac du rostre dépassant largement le bord postérieur des ventouses : $120 \times 60 \mu$. Une couronne de 10 crochets claviformes de 32 à $44 \mu$ de long, à manche et garde bien développés (fig. 64 et 65 ).

Cou : $250 \times 80-100 \mu$ de diamètre.

ANNEAUX: de petite taille $(160 \times 32 \mu$ pour un anneau mûr), plus larges que hauts, cylindriques. Protérandrie accusée; les testicules ne persistent pas dans les anneaux femelles; un grand nombre d'anneaux ne présentent pas encore de glandes femelles formées, alors que les testicules ont disparu depuis longtemps; les réservoirs séminaux envahissent tout le parenchyme médullaire.

\section{A) Système mâle.}

ANNEAU MâLE : $100-120 \times 20 \mu$ environ (fig. 66 et 67 ).

Testicules : disposés suivant le type B ou C. Petits : $23 \times 8 \mu$.

VÉsicule SÉminale EXTERNE: dorsale, située au niveau du fond de la poche du cirre. Ovoïde. De $26 \times 13$ à $50 \times 25 \mu$ suivant sont état de réplétion; vient se situer dans le prolongement de la poche du cirre dans les anneaux gravides mûrs. Le canal déférent intervésiculaire est très dilaté et participe à la mise en réserve des spermatozoïdes.

POCHE DU CIRRE : relativement grande, musculeuse, fusiforme : $65-75 \times 18-20 \mu$ de diamètre; atteint ou franchit les C.O.R. antiporaux. Pas de fibres rétractrices visibles. Cirre petit, ponctué finement, très difficilement visible (10-15 $\mu$ de long). $\mathrm{PC} /$ $\mathrm{AM}=1 / 2$ à $1 / 1,5$.

B) Système femelle. (fig. 68).

OvaIRE : ventral, médian, trilobé, $26 \times 50 \mu$.

Vitellogène : ventral par rapport à l'ovaire. $13 \times 26 \mu$. Ovoïde.

RÉCEPTACLE SÉminAL : relativement petit. $13 \mu$ de diamètre. Ovoïde. Situé entre la poche du cirre et l'aile porale de l'ovaire.

VAGIN : invisible; absence de sphincter et de fibres rétractrices apparentes.

UTÉrus : sacculaire, bi-lobé à l'état jeune, puis ultérieurement multi-lobé. Contient un grand nombre d'œufs. Les anneaux gravides mûrs mesurent $250 \times 35-45 \mu$ de haut (fig. 69).

CEufs MûRS : nettement ovoïdes, à coque uniformément mince. Coque externe : 25-30 $\mu \times 15-20 \mu$ de diamètre. Coque interne : $20 \mu$ de long $\times 9 \mu$ de diamètre. Embryon hexacanthe allongé $17 \times 8 \mu$, à crochets de 5-6 $\mu$ de long (fig. 65). 


\section{Diseussion.}

H. rectacantha Fuhrm., 1906 de Charadrius hiaticula (L.) du Groenland, sommairement décrite à l'origine d'après des exemplaires incomplets, et très médiocrement illustrée, demeure énigmatique. L'hypothèse aue $H$. cambrensis Davies, 1939 lui est synonyme ne peut plus malheureusement être vérifiée (les exemplaires d'après lesquels Davies l'a redécrite en $1939^{\circ}$ ne figurent plus dans les collections de cet auteur à Aberystwyth. Communication personnelle du $D^{r}$ F. G. Rees de 1962). Les crochets du cestode de Fuhrmann, rectilignes (fig. 27, p. 447) comme la dénomination le spécifie, ne ressemblent pas à ceux, tout en sinuosités au contraire et à garde bien accusée, de l'espèce groenlandaise recueillie par Baer chez l'huîtrier pie; les testicules n'y sont pas en ligne, mais en triangle. Ce sont les raisons qui motivent, à notre avis, la création d'une espèce nouvelle, au moins provisoirement. Son habitus d'Aploparaksis que rappellera son nom spécifique, ses crochets, la petitesse de son cirre, l'individualise facilement parmi toutes les autres espèces d'Hymenolepis parasites de Charadrii.

\section{HYMENOLEPIS (HYM.) RYBICKAE n. sp.}

Syn.: H. capellae sensu Rybicka, 1958, nec Baer, 1940.

Région paléarctique: Pologne (Lac Druzno).

Hôte : Capella gallinago (L.).

Matériel observé : Préparation de K. Rybicka contenant un strobile mûr.

Description de l'espèce (planche 16, fig. 71 à 75 ).

Strobile: long de 40 à $50 \mathrm{~mm} \times 0,75$ de largeur maximale au niveau des anneaux sexuellement mûrs. Formé de 400 anneaux environ: 150 très immatures (anneaux de cou), 60 immatures, 60 mâles, 40 hermaphrodites, 80 anneaux gravides au moins.

Pores génitaux : unilatéraux, dextres.

Atrium génital: dépourvu de sacculus et d'épines; long d'une centaine de microns.

Musculature longitudinale interne formée de quatre larges faisceaux par face ( $25 \mu$ à $40 \mu$ de diamètre).

C.O.R. : les C.O.R. se situent ventralement par rapport aux conduits génitaux. Les vaisseaux ventraux sont larges, les dorsaux plus apparents et plus fins $(5 \mu$ de diamètre).

Scolex : Le seul scolex observé est assez déformé ; il semble morphologiquement s'apparenter à celui d' $H$. amphitricha (Rud.); ses dimensions approximatives seraient $130 \mu$ de long $\times 100 \mu$ de diamètre. Ventouses : $60 \mu$ de diamètre, inermes. Rostre demi-évaginé : $90 \mu$ mesurés jusqu'au fond du sac $\times 23 \mu$ de diamètre ; garni d'une couronne de 10 crochets chéliformes de 16-17,5 $\mu$ de long $\times 6,5-7,5 \mu$ de hauteur, à lame très effilée nettement plus longue que la garde (fig. 74). 
Cou : court, $100 \times 100 \mu$ environ.

ANNEAUX: trapézoïdaux, beaucoup plus larges que hauts (rapport hauteur/largeur $=1 / 7$ environ). Il y a protérandrie et les testicules persistent dans les anneaux femelles mûrs.

\section{Appareil reproducteur.}

\section{A) Appareil mâle.}

Testicules: toujours en ligne (type G). Volumineux: 130-150 $\times 70-80 \mu$; adjacents les uns aux autres dans un même anneau, ils dépassent souvent latéralement les C.O.R. dorsaux (fig. 71).

VÉSICULE SÉminAle EXTERNE : reliée à la poche du cirre par un canal intervésiculaire de $60-80 \mu \times 8-10 \mu$ de diamètre gui l'aborde souvent du côté antiporal, elle se situe dans le prolongement de la poche du cirre, au niveau du testicule antiporal; elle dépasse parfois latéralement les C.O.R. antiporaux. Sphérique (100 $\mu$ de diamètre) ou ovoïde $($ de $100 \times 50$ à $160 \times 50 \mu)$.

POCHE DU CIRRE : droite et parallèle au bord antérieur de l'anneau. Parois musculaires minces; son fond dépasse l'axe longitudinal du strobile dans les anneaux mâles atteignant presque les C.O.R. antiporaux. Elle mesure 290-340 $\mu \times 50 \mu$ de diamètre dans des anneaux mâles de $600 \times 100 \mu, 340 \times 50$ dans les anneaux mûrs hermaphrodites de $700 \times 100 \mu$. Le rapport $\mathrm{PC} / \mathrm{AM}$ est égal à $1 / 2,4$. Elle atteint jusqu'à $365 \mu$ dans les anneaux gravides. Elle contient une vésicule séminale interne particulièrement courte, égale au 1/3 environ de sa longueur totale, piriforme; le canal éjaculateur qui la prolonge décrit une boucle avant de se différencier en un très long canal de 13-16 $\mu$ de diamètre, sinueux, qu'individualisent bien ses parois épaissies $(2,5 \mu)$. L'état de fixation du Cestode ne permet pas de distinguer s'il s'agit de soies ou d'une ornementation d'un autre type (9); la portion terminale, longue de $90 \mu$ environ, légèrement plus dilatée $(15-20 \mu)$ est garnie d'épines fines de 2 à $3 \mu$ (fig. 73).

Le CIRRE évaginé est puissant, cylindrique. Il mesure jusqu'à $200 \mu$ de long sur un diamètre de $30 \mu$ à la base, légèrement renflée, située à l'intérieur de l'atrium génital sur une longueur de $60 \mu$ environ et armée d'épines de 2-3 $\mu$. Le diamètre distal est de 20-22 $\mu$; une ornementation existe, mais sa nature ne peut être précisée.

\section{B) Appareil femelle.}

Le massif des glandes femelles apparaît dans les anneaux mâles au niveau du testicule médian (fig. 71 et 72 ).

OVAIRE: très vaste à maturité complète, il occupe ventralement presque tout l'espace situé entre les C.O.R. longitudinaux. Il mesure 190 à $240 \mu$ de large. Nettement trilobé ; chacun des lobes, à peu près égaux, mesurent $100 \times 80 \mu$ de hauteur environ.

VITELlogènE : ventral et postérieur à l'ovaire, de 90-100 × 40-55 $\mu$ de haut, il forme une masse légèrement lobée.

(9) Elle apparaît comme de fines villosités de 2,5 u, mais il pourrait s'agir de soies, d'épines, ou de verrucosités altérées. 
RÉCEPTACLE SÉminAL: il se situe en avant de l'aile porale de l'ovaire, ne fran-

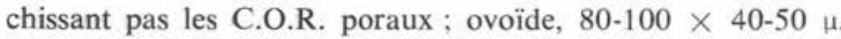

VAGIN : sa morphologie l'apparente à celle de $H$. lauriei Davies. Très long (250 à $300 \mu$ ), il est uniformément tubulaire, sans différenciation distale copulatrice ni de dilatation ampullaire et décrit de grandes sinuosités. Sa paroi interne de $10 \mu$ de diamètre, légèrement chitinisée, apparaît finement plissée longitudinalement ; les coupes optiques montrent ces plis comme des villosités. Ce tube est entouré d'un manchon discret de cellules qui porte son diamètre externe à $20 \mu$ environ. Il ne présente ni sphincter ni fibres rétractrices (fig. 73).

UTÉRUs : invisible dans les anneaux sexuellement mûrs. Dans les anneaux gravides, il est sacculaire, bilobé et contient un grand nombre d'œufs. Les anneaux gravides s'allongent sans s'élargir et mesurent $800 \times 310 \mu$. Y persistent bien apparents : $1^{\circ}$ la poche du cirre, la vésicule séminale externe qui se situent un peu au-dessus de l'axe horizontal de l'anneau. $2^{\circ}$ le vagin souvent dirigé obliquement en direction du réceptacle séminal qui se dispose très postérieurement dans la concavité de l'utérus (fig. 75).

EUfs: insuffisamment mûrs pour fournir une description utilisable. Il semble qu'ils doivent être sphériques ou légèrement ovoïdes et mesurent au moins $30 \times 45 \mu$ ou $40 \times 40 \mu$.

\section{Discussion.}

L'examen comparé des types de $H$. capellae Baer, 1940 et des exemplaires de Rybicka montre qu'il s'agit en réalité de deux espèces distinctes, ainsi que nous le supposions en 1962 (p. 778). La position testiculaire, les anatomies du cirre et du vagin ne se confondent pas. Aucune autre espèce déjà décrite d'Hyménolepis de Charadriiforme, pourvue de crochets chéliformes ou non, ne réunit simultanément les caractères des conduits génitaux énumérés dans la description ci-dessus; nous la considérerons donc comme nouvelle et la dédierons à K. Rybicka sous le nom de Hymenolepis (Hym.) rybickae n. sp.

\section{D) ESSAI DE CLE DIAGNOSTIQUE}

Celle de 1962 (p. 839-843) n'est pratiquement plus valable; nous en fournissons une version modernisée tenant compte des synonymies nouvelles et de nos observations, utilisant les mêmes abréviations, et basées sur le maximum de caractères spécifiques. N'y figurent pas $H$. porale Meggitt au scolex inconnu et $H$. longiovata Johri non encore décrite.

Rostre dépourvu de crochets. Scolex globuleux. Testicules du type B. Cirre épineux de $100 \mu$. Vagin à papilles. 20 B.M.I. Parasite du rectum . . . . . . . . . . . .

H. (H.) glandularis Fuhrm.

Rostre pourvu de crochets :

Crochets chéliformes (= Aploparaksiformes) 
Crochets falciformes (rapport longueur du manche/longueur de la lame voisin

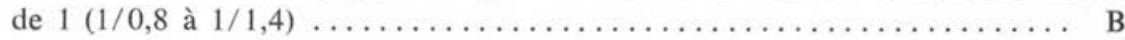

Crochets claviformes (rapport longueur du manche/longueur de la lame très différent de 1 ; le manche $=2$ à 5 fois la lame)

\section{A) Crochets chéliformes}

Une couronne de $68-70 \mathrm{cr}$. de 9-12 $\mu$. P. du C. de $147 \mu . \mathrm{PC} / \mathrm{AM}=1 / 4 \ldots \ldots \ldots$ H. (H.) skriabinissima Krotov

Une couronne de 10 crochets

1. . Ventouses typiques d'Hymenolepis. Atrium génital non épineux. Présence d'un petit sac accessoire du type $*$ peigne atrial $\$ \ldots \ldots \ldots \ldots \ldots \ldots \ldots \ldots 2$

Idem, mais absence de sac accessoire ..................... 3

2. . Crochets de $8 \mu$. P. du C. de 180-200 $\mu(\mathrm{PC} / \mathrm{AM}=1 / 3)$. Cirre de 20-30 $\mu$

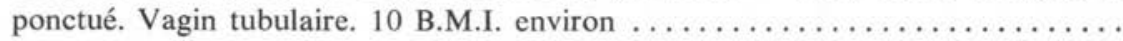

H. (H.) kwangensis Southwell et L.

3. . Cr. du rostre inférieurs à $20 \mu(8$ à $18 \mu)$. Testicules toujours du type $\mathrm{G}$ (en

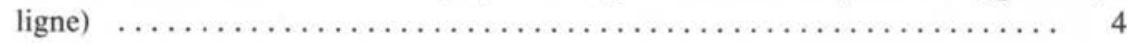

Cr. du rostre supérieurs à $20 \mu$ (20-47). Testicules du type B ou du type G. . 9

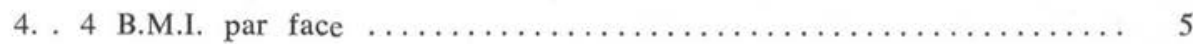

Nombre de B.M.I. supérieur à 4 (15 minimum). Atrium génital toujours dépourvu

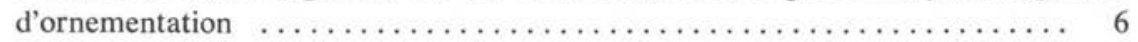

5. . Cul-de-sac atrial en forme de cuvette verruqueuse. Cr. de $9 \mu$. Test. non persistants. $\mathrm{PC} / \mathrm{AM}=1 / 2,5$ à $1 / 5$. Cirre cylindrique glabre de 50-90 $\mu$. Vagin conique entouré d'un épais manchon glandulaire, tapissé de papilles, avec gros sphincter et fibres rétractrices .......... H. (H.) tsengi Joyeux et Baer Atrium génital tubulaire dépourvu de verrues. Cr. de 16-17 $\mu$. Testicules persistants. $\mathrm{PC} / \mathrm{AM}=1 / 2,4$. Cirre cylindrique de $200 \mu$ armé d'épines de $2-3 \mu$. Vagin membraneux, tubulaire, sinueux, de 250-300 $\mu$, sans sphincter ou fibres rétractrices $\ldots \ldots \ldots \ldots \ldots \ldots \ldots \ldots \ldots \ldots \ldots \ldots \ldots \ldots \ldots \ldots \ldots \ldots \ldots$ H. (H.) rybickae n. sp.

6. Vagin distal occlus d'un gros sphincter muni de fibres rétractrices puissantes. Réceptacle séminal ovoïde, $\mathrm{PC} / \mathrm{AM}=1 / 2$ à $1 / 3$. Cirre court ponctué. Crochets

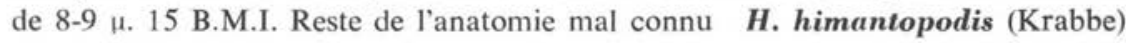
Vagin dépourvu de sphincter et de fibres. Cr. de 10-15 $\mu$. Testicules toujours en

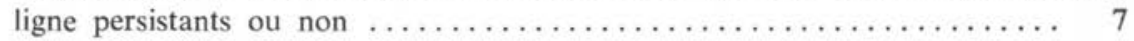

7. Testicules non persistants (protérandrie nette). $\mathrm{PC} / \mathrm{AM}=1 / 2$ à $1 / 3$. Cirre de $100 \mu$ armé. Vagin chitinoïde de $50 \mu$ environ. R.S. ovoïde ne franchissant pas les C.O.R. poraux. 20 à 30 B.M.I. Cr. de 9 à $15 \mu$ H. recurvirostrae Krabbe Testicules persistants, EN ARRIÈrE de l'ovaire. Cr. de 13-14 $\mu$. Vagin membraneux, simplement tubulaire de 60 à $100 \mu \ldots \ldots \ldots \ldots \ldots \ldots \ldots \ldots . . . \ldots$ 
8. . P. du C. courte $(190 \mu$ en moyenne). $\mathrm{PC} / \mathrm{AM}=1 / 5$ ou $1 / 6$. Cirre court $(35 \mu)$, inerme. R.S. piriforme franchissant les C.O.R. poraux :

a) Strobile de 25-30 mm, cirre court, ampullaire, porté par un col rétréci.

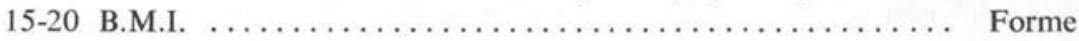
tri-testiculée (avec souvent 4 testicules) d'Oligorchis paucitesticulatus (Fuhrm.)

b) Strobile de $80 \mathrm{~mm}$. Cirre cylindro-conique $(25-35 \mu), 15-20$ B.M.I. . . . . . .

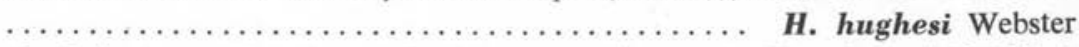
P. du C. longue, 290-340 $\mu(\mathrm{PC} / \mathrm{AM}=1 / 3,5$ à 1/4,5). Cirre long $(130-160 \mu)$ soyeux, bien visible, même invaginé. R.S. ovoïde, ne franchissant pas les C.O.R. poraux ................ H. longocylindrocirrus Deblock et Rosé

9. . P. du C. courte $(\mathrm{PC} / \mathrm{AM}=1 / 8)$. Crochets de $30 \mu$. Testicules persistants en triangle (type B). Cirre court glabre. R.S. piriforme. Vagin membraneux court $(80 \mu) .40$ B.M.I. .................. annandalei Southwell P. du C. longue $(\mathrm{PC} / \mathrm{AM}=1 / 2$ à 1/3). Cirres de plus de $100 \mu \ldots \ldots \ldots 10$

10. . Test. en ligne. Cr. de 28-30 $\mu$ ou 40-47 $\mu$. P. du C. de $400 \mu(\mathrm{PC} / \mathrm{AM}=1 / 2,3)$.

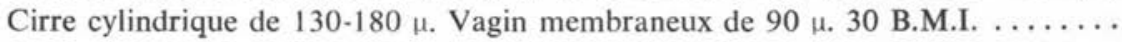
$\ldots \ldots \ldots \ldots \ldots \ldots \ldots \ldots \ldots \ldots \ldots \ldots \ldots \ldots \ldots \ldots \ldots$. clandestina (Krabbe)

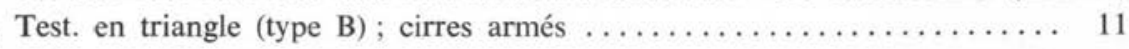

11. . Cirre puissant $(150-200 \mu)$ armé de fortes épines. P. du C. de $400 \mu$ (PC/ $\mathrm{AM}=$ 1/3,5). Vagin chitinoïde de $220 \mu$. Crochets de 20-23 $\mu$ et $33 \mu$. 10-15 B.M.I. $\ldots \ldots \ldots \ldots \ldots \ldots \ldots \ldots \ldots \ldots \ldots \ldots \ldots \ldots \ldots \ldots \ldots \ldots$. amphitricha (Rud.) Cirre cylindrique soyeux de $140 \mu$. P. du C. de $240 \mu(\mathrm{PC} / \mathrm{AM}=1 / 2)$. Vagin chitinoïde de $90 \mu$. Crochets de $23-24 \mu \ldots \ldots \ldots \ldots \ldots$. capellae Baer

弯

N. B. - 2 species inquirendae :

- Cr. de 21-23 $\mu$ à garde massive. P. du C. courte $(180 \mu) \mathrm{PC} / \mathrm{AM}=1 / 4$ à $1 / 5$ (?) $\ldots \ldots \ldots \ldots \ldots \ldots \ldots \ldots \ldots \ldots \ldots \ldots \ldots \ldots \ldots \ldots h$ chionis Fuhrm

- Cr. de $42-45 \mu$ voisins de ceux de clandestina, mais strobile court (4 $\mathrm{mm}$ au lieu

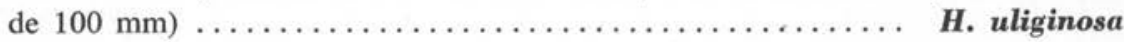

\section{B) Crochets falciformes}

Dans la clé de 1962, p. 841, seuls les reports $\mathrm{n}^{\circ *} 1$ et 2 correspondant aux Echinocotyle sont à modifier. Pour le reste, tenir simplement compte de la synonymie de $H$. charadrii avec $H$. nitidulans et de $H$. magnisaccis avec $H$. glareolae.

Ventouses typiques d'Echinocotyle. S.A. toujours présent. Atrium armé. Cirre glabre. Petits strobiles, de longueur inférieure à $5 \mathrm{~mm} \ldots \ldots \ldots \ldots \ldots .1$ 
1. S.A. hypertrophié proportionnellement à l'anneau, garni de 10 fortes épines de

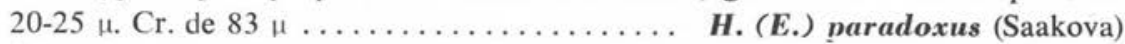
S.A. plus proportionné ou petit $(40 \mu)$, garni de nombreuses petites épines de

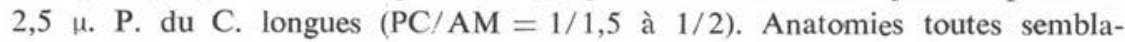

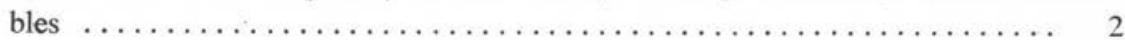

2. Crochets de $33 \mu \ldots \ldots \ldots \ldots \ldots \ldots \ldots \ldots$ H. (E.) longirostris (Rud.) Crochets de $65-67 \mu \ldots \ldots \ldots \ldots \ldots \ldots \ldots$ H. (E.) magnisaccis (Meggitt) Crochets de $86-89 \mu \ldots \ldots \ldots \ldots \ldots \ldots \ldots$ H. (E.) dubininae (Clerc)

\section{C) Crochets claviformes}

Ventouses typiques d'Echinocotyle. S.A. toujours présent. Atrium armé. Cirre glabre cylindro-conique. Vagin membraneux, tubulaire, court $\ldots \ldots \ldots \ldots . \quad 1$ Ventouses totalement inermes. Absence de S.A.

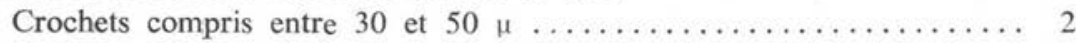

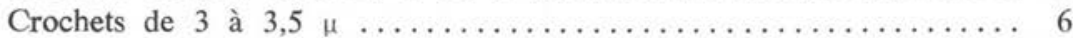

1. . Cr. de 20-25 $\mu, 2 \times 4$ B.M.I. ; P. du C. courte $(100-150 \mu) . \mathrm{PC} / \mathrm{AM}=1 / 5$ à $1 / 6$. Testicules du type $\mathrm{C} \ldots \ldots \ldots \ldots \ldots \ldots \ldots \ldots \ldots \ldots$. (E.) tenuis Clerc

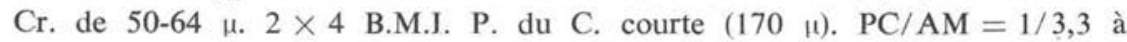
$1 / 8,5$. Testicules du type $\mathrm{D}$ ou $\mathrm{H}$ (suivant contraction). Ovaire très large. Vtg

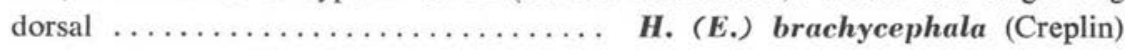

2. . Cestodes très petits ou petits $(5-25 \mathrm{~mm})$, à $\mathrm{P}$. du $\mathrm{C}$. « longues » $(\mathrm{PC} / \mathrm{AM}=1 / 1,5$

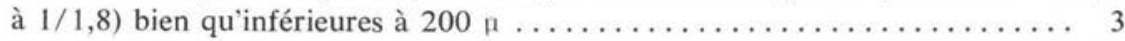
Cestodes moyens $(40-100 \mathrm{~mm})$, à $\mathrm{P}$. du C. * courtes $(\mathrm{PC} / \mathrm{AM}=1 / 2$ à 1/4) bien que supérieures à $270 \mu$. Testicules non en ligne (type $\mathrm{C}$ ou $\mathrm{D}$ ) $\ldots \ldots \ldots 5$

3. Testicules non persistants, en ligne (type G). Crochets rectilignes de 46-51 $\mu$. Poche du cirre de $160 \mu$. Cirre inerme court $(25 \mu)$. Vagin membraneux de $40 \mu$, pelotonné dans la région atriale .......... H. (H.) cambrensis Davies Testicules non persistants, toujours en triangle (type B ou C). Crochets inférieurs à $45 \mu$. P. du C. de 70-80 $\mu$. Cirres de 10-15 $\mu$ très finement armés

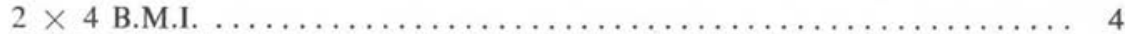

4. . Cr. sinueux de $32-44 \mu$. Cirre cylindrique ponctué. Vagin membraneux (?) peu visible. Eufs mûrs nettement ovalaires de $27 \times 18 \mu \ldots \ldots \ldots \ldots \ldots \ldots \ldots$

H. (H.) aploparaksioidis n. sp. Cr. rectilignes de 40-43 $\mu$. Cirre de $15 \mu$ armé de petits crochets au niveau d'une ampoule basale. Vagin distal cylindro-cônique, court $(20 \mu)$, chitinoïdisé

H. (H.) rectacantha Fuhrm.

5. . $2 \times 25-30$ B.M.I. Huit crochets de $35-44 \mu$. Vagin très développé $(140 \times 40 \mu)$ à papilles, entouré de cellules glandulaires. Cirre très long $(200 \mu)$. Testicules du 
type B ou C . . . . . . H. (H.) vaginata Baczynska (10) $2 \times 4$ B.M.I., 10 cr. de 32-35 $\mu$. Vagin membraneux tubulaire sans papille, de $80 \mu$. Cirre soyeux de $260 \mu$. Testicules du type D . . . . . . . . . . . . . . $\ldots \ldots \ldots \ldots \ldots \ldots \ldots \ldots \ldots \ldots \ldots \ldots \ldots \ldots \ldots \ldots \ldots \ldots$ H. (H.) interrupta (Rudolphi)

6. Strobile de 5-7 $\times 0,6-1,1 \mathrm{~mm}$. Testicules en ligne. $\mathrm{PC} / \mathrm{AM}=1 / 2$. Cirre inerme. Utérus gravide bisacculé. CEufs de $20 \times 25 \mu$ de diamètre $\ldots \ldots \ldots \ldots \ldots \ldots$

H. (H.) jerrata Johri.

\section{E) CONCLUSIONS}

Ayant poursuivi l'examen des types des espèces d'Hymenolepis parasites de Charadriiformes commencé en 1962, il nous a été possible de confirmer plusieurs des hypothèses que nous avions émises concernant :

A) soit l'hétérogénéité de certaines espèces transparaissant dans les descriptions de la littérature :

$1^{\circ}$ Le complexe annandalei, dissocié en $H$. clandestina et Aploparaksis sp.

$2^{\circ}$ Le complexe recurvirostrae, dissocié en quatre espèces différentes: recurvirostrae sensu stricto; himantopodis sensu Meggitt ; tsengi (J. et B., 1940) qui groupe toutes les espèces décrites à ce jour sous le nom d' $H$. himantopodis, à l'exception de l'espèce-type de Krabbe ; et Aploparaksis sp.

$3^{\circ} H$. capellae, dont nous distinguons $H$. rybickae n. sp.

B) Soit au contraire certaines analogies (synonymies de $H$. interrupta et de H. nitidulans notamment).

D'autres synonymies, absolument imprévisibles dans l'état de nos connaissances antérieures, se sont clairement manifestées.

Beaucoup de types ont été redécrits avec la précision compatible avec l'état des préparations observées.

En dépit de la création de 7 espèces nouvelles depuis 1962 (belopolskaïae, capetownensis, etaplesensis, patersoni ; longocylindrocirrus, aploparaksiö̈dis, rybickae), les 54 espèces d'Hymenolepis parasites de Charadriiformes que comptait la littérature en 1962 ont été réduites au nombre de 40 par le jeu des synonymies ou par exclusion du genre (11).

Seules demeurent species inquirendae: $H$. chionis, paranitidulans, rectacantha,

(10) Depuis la rédaction de ce travail, il vient s'intercaler, à peu près à ce niveau de notre clé, Hymenolepis (H.) megacantha (Spassky et coll., 1963) avec les caractéristiques essentielles suivantes: strobile de 15 à $20 \mathrm{~mm} \times 0,46$. Scolex de $300 u$ de diamètre. 10 crochets claviformes de $90-95 \mu$ (la longueur du manche égale deux fois la lame). Ventouses inermes. Nombre de B.M.I. supérieur à 4. Disposition ovaire-testicule du type B.P. du C. de $126 \times 38 \mu$ (dans les anneaux de $460 \times 310 \mu$ ). $\mathrm{PC} / \mathrm{AM}=1 / 4$. Cirre et vagin non décrits. Anneaux gravides inconnus. Hôte : Tringa sp. de la région indienne (Viêt-Nam).

(11) $H$. longiovata Johri n'ayant pas encore fait, à notre connaissance, l'objet d'une publication ne peut être considérée comme valide. 
uliginosa, ainsi que porale dont le scolex n'est pas connu. Le quart des espèces décrites est présent en France (amphitricha, brachycephala, etaplensensis, lauriei, longocylindrocirrus, magnisaccis, nitida, nitidulans; clandestina, longirostris et tsengi); les huit premières ont été trouvées sur les côtes de la Manche.

Le genre Oligorchis déjà amoindri de ses Paradilepis, se voit encore réduit par la perte de ses Hymenolepis (kwangensis et toxometra) à 3 espèces parasites d'Oiseaux : strangulatus, paucitesticulatus et cyanocittii.

Il se confirme en outre que les caractères spécifiques des Hymenolepis sont nettement marqués, dans la plupart des cas, au niveau des terminaisons des conduits génitaux mâles et femelles. En conséquence, les auteurs découvrant de nouvelles espèces doivent les définir essentiellement par la description et l'illustration détaillée de ces organes, examinés aux grossissements les plus puissants des microscopes (immersion). Le désordre qui règne actuellement dans la systématique de groupes de Cestodes réputés confus semble avoir été créé bien artificiellement par des examens hâtifs conduisant à des descriptions superficielles donc approximatives, sinon inexactes.

Lorsqu'un pareil travail de révision aura été entrepris pour tous les Hymenolepis connus, peut-être sera-t-il possible de préciser les affinités morphologiques de certaines espèces, que l'on pourra alors grouper en sous-genres homogènes ; néanmoins, d'après ce que nous connaissons actuellement de la morphologie des Hymenolepis de Charadriiforme, il semble que cette voie ne laisse subsister qu'une très problématique espérance d'aboutissement.

\section{Bibliographie}

Ne figurent ici que les références nouvelles par rapport à l'article de Deblock et Rosé de 1962 ou venant en complément de celui-ci.

BAER (J. G.), 1962. - The Zoology of Iceland II (12). Cestoda, E. Munksgaard, Copenhague et Reykjavik, édit.

CZAPLINSKI (B.), 1956. - Hymenolepididae Fuhrmann, 1907 (Cestoda), parasites of some domestic and wild Anseriformes in Poland. Acta. Parasit. Polon., 4 (8), p. 175-373.

Deblock (S.) et Rosé (F.), 1962. - Les Hymenolepis (sensu lato) de Charadriiformes (à propos de 23 descriptions). Ann. Par. hum et comp., 37 (5-6), p. 767-847.

- - 1964. - Hymenolepididae (Cestoda) des Charadriiformes des Côtes de France. Validité du genre Oligorchis Fuhrm., 1906 et description d'Hymenolepis longocylindrocirrus n. sp. Ann. Par. Hum. et Comp., 39, p. 157-178.

JARECKA (L.), 1958. - Plankton crustaceous in the life cycle of tapeworms occuring at Druzno Lake. Acta Parasit. Polon., 6 (2), p. 65-109.

JCHRI (L. N.), 1960, - On two new avian Cestodes belonging to the subfamily Hymenolepididae Perrier, 1897 from Delhi State. Proceed. Nat. Acad. of Sciences, India, sect. B, part III, 30 , p. $234-240,2$ fig. 
Joyeux (C.), Gendre (E.) et BAer (J. G.), 1928. - Recherches sur les helminthes de l'Afrique Occidentale française. Coll. de la Soc. de Path. Exot. Monographie 11. Masson et $\mathrm{C}^{\text {ie }}$ édit. Paris, $120 \mathrm{p}$.

KoRPACZEWSKA (W.), 1959. - The adult forms of Echinocotyle druzniensis Jarecka, 1958. Bull. Acad. Polon. des Sciences, 7 (5), p. 195-198.

Macko (J. K.), 1962. - Revízia druhu Hymenolepis oweni Moghe 1933, Nájdeného u novych hostitel'ov, a jeho preradenie do rodu Echinocotyle Blanchard, 1891. Biológia (Bratislava), 12 (8), p. 606-613.

MetrricK (D. F.), 1958. - Helminth Parasites of Hertfordshire birds. II. Cestoda. Jl. of Helminth., 32 (3), p. 159-194.

Ryвicka (K.), 1958. - Cestodes d'Oiseaux (Ansériformes exceptés) du Lac Druzno (Faune parasitaire de la biocénose du Lac Druzno. IV ${ }^{e}$ partie). Acta Parasit. Polon., 1958, 6 (4), p. 143-178.

SingH (K. P.), 1959. - Some avian Cestodes from India. III. Species belonging to family Hymenolepididae. Indian Jl. of Helminth., 11 (1), p. 43-62.

Southwell (T.) et LAKe (F.), 1939. - On a collection of Cestoda from the belgian Congo. Ann. Trop. Med. Parasit., 33, p. 63-90 et 107-123.

SPASSKY (A.-A.), 1963. - Eléments de cestodologie. Tome II : les Hyménolépididés. Cestodes d'oiseaux domestiques et sauvages ( $1^{\text {re }}$ partie). Edition de l'Académie des Sciences de l'U.R.S.S., Moscou, 1963, 418 pp. (p. 226-234).

Spassky (A. A.), Dang (V. N.) et Jurpalova (N. M.), 1963. - Trois nouvelles espèces d'Hymenolepididés d'oiseaux domestiques et sauvages du Viêt-Nam, in Parasites des Animaux et des Végétaux de Moldavie. Ed. de I'Inst. Biol. Acad. Sci. Rép. Moldave (133 p.), p. 75-83, 8 fig. (en russe).

Tubangui (M. A.) et Masiluñgan (V. A.), 1937. - Tapeworms parasites of Philippine birds. Philippine Jl. of Science, 62 (4), p. 409-438.

Williams (I. C.), 1961. - A list of parasitic worms including twenty-two new records from british birds. Ann. Mag. of Nat. Hist., ser. 13, 4, p. 467-480.

Travail du Laboratoire de Parasitologie

de la Faculté mixte de Médecine-Pharmacie de Lille (France) $\left(\mathrm{D}^{\mathrm{r}}\right.$ : $\mathrm{P}^{\mathrm{r}} \mathrm{J}$. BigueT)

et de l'Institut de Zoologie de Neuchâtel (Suisse) ( $\mathrm{D}^{r}$ : $\mathrm{P}^{r}$ J. G. BAER) 

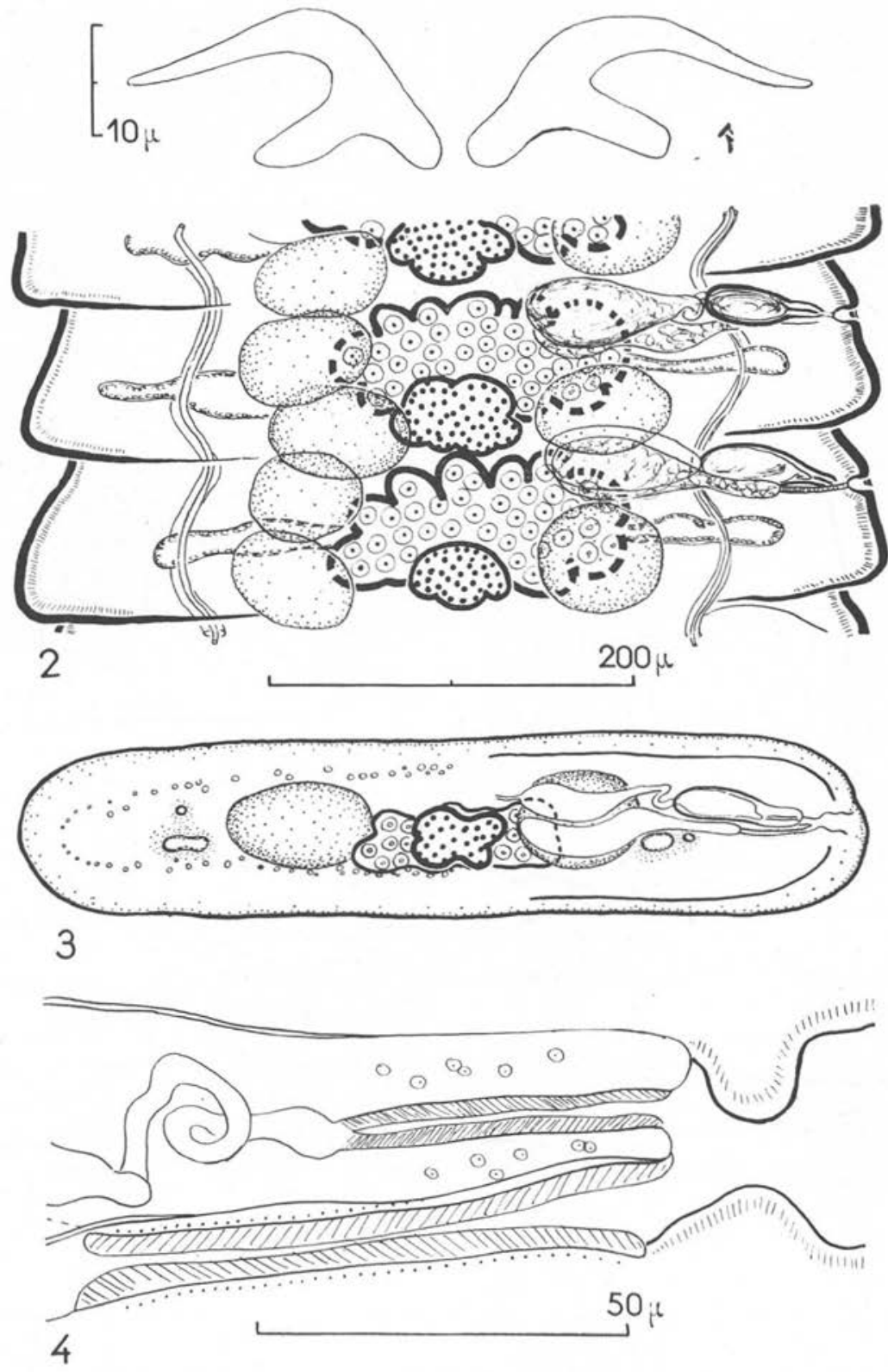

Planche 1. - H. (H.) annandalei Southwell, 1922. Types. 1. Crochets. 2. Anneau mûr (vue dorsale). 3. Coupe transversale d'anneau mûr. 4. Détail des conduits génitaux (Coupe histologique). 

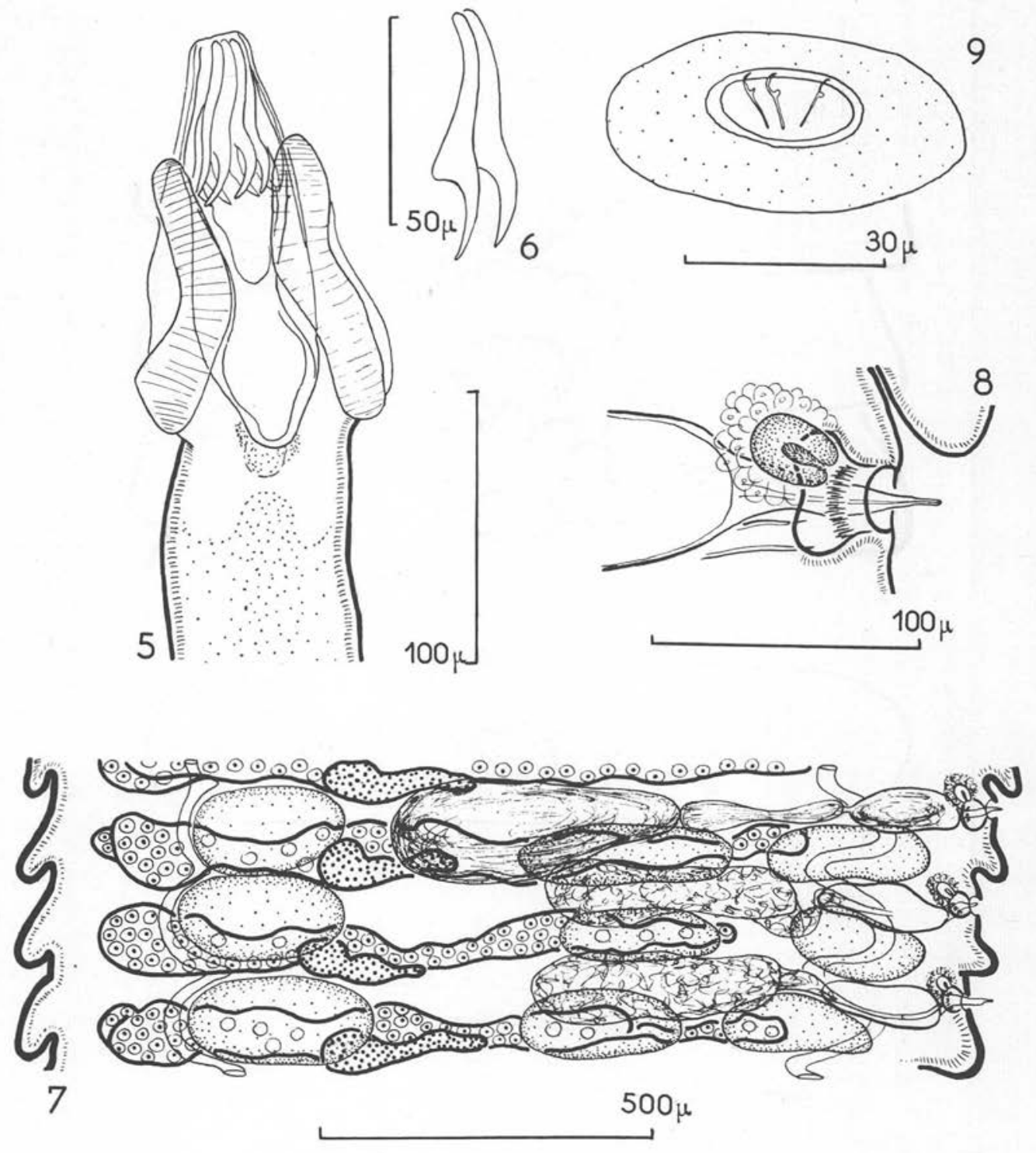

Planche 2. $-H$. (E.) brachycephala (Créplin, 1829). Exemplaires de Machetex pugnix (Dombes, France) in Joyeux et Baer, 1936. 5. Scolex (profil). 6. Crochets. 7. Anneaux mûrs (vue dorsale). Des organes ont été omis dans les anneaux pour la clarté du dessin. 8. Atrium génital. Vue dorsale 9. CEuf mûr. 


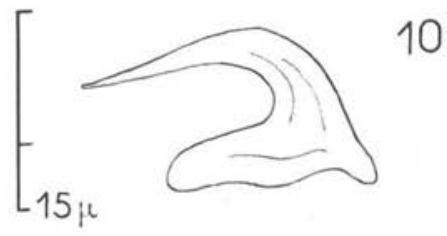

10

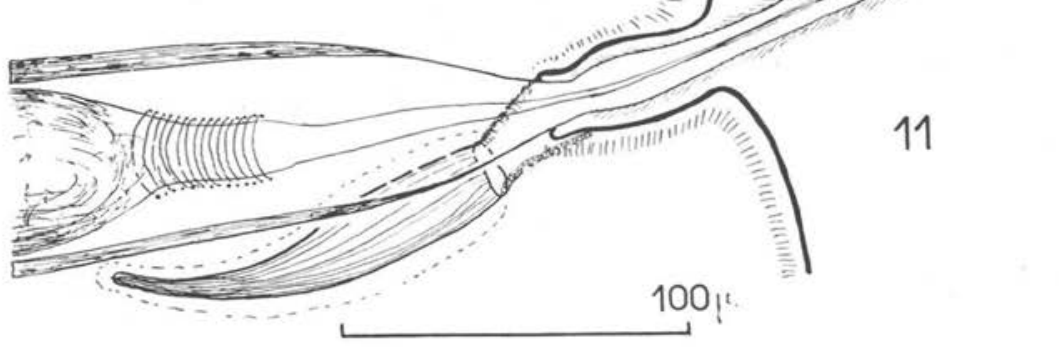

Planche 3. $-H$. $(H$.$) capellae Baer, 1940. Type. 10. Crochets. 11. Terminaisons géni-$ tales of et $q$.
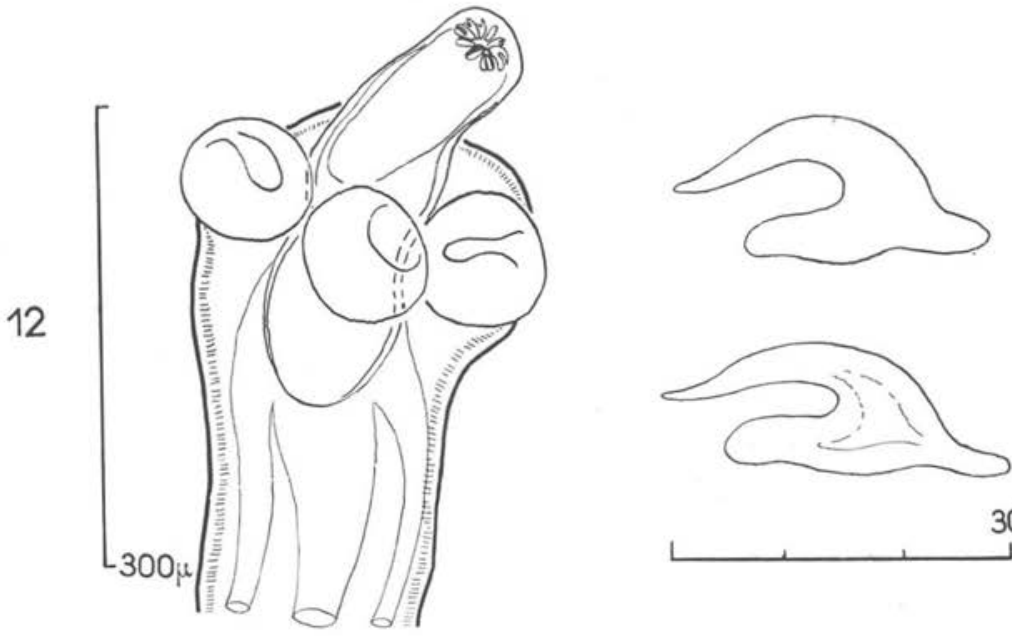

Planche 4. - Légende page suivante. 


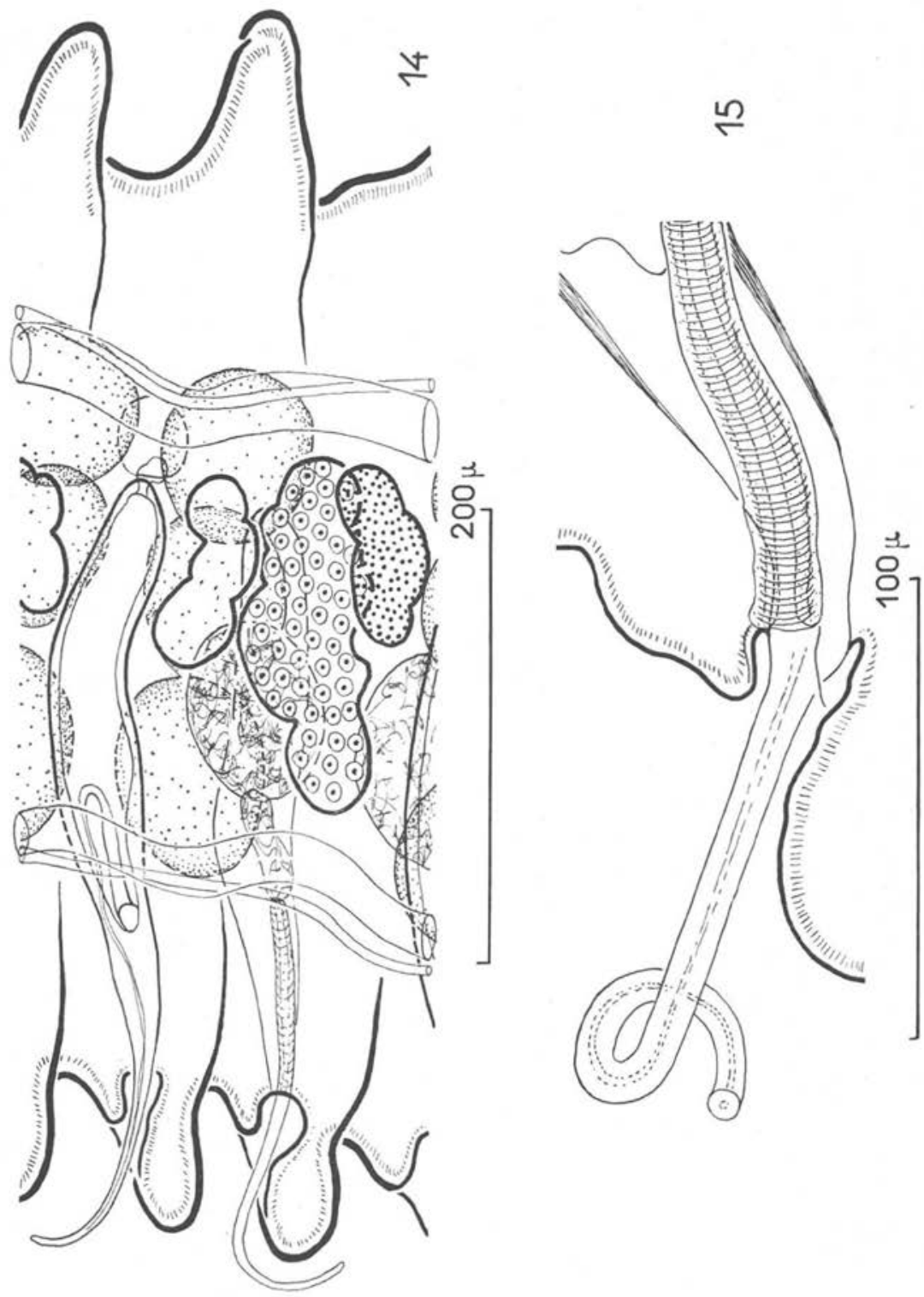

Planche 4. $-H$. $(H$.$) clandestina (Krabbe, 1869). Exemplaires de Tringa sp. (Midi$ de la France). Joyeux et Baer, 1939. 12. Scolex. 13. Crochets. 14. Anneaux mûrs. Vue ventrale. (Des organes ont été omis dans les anneaux pour la clarté du dessin). 15. Terminaisons génitales $\hat{\sigma}$ et ?. 

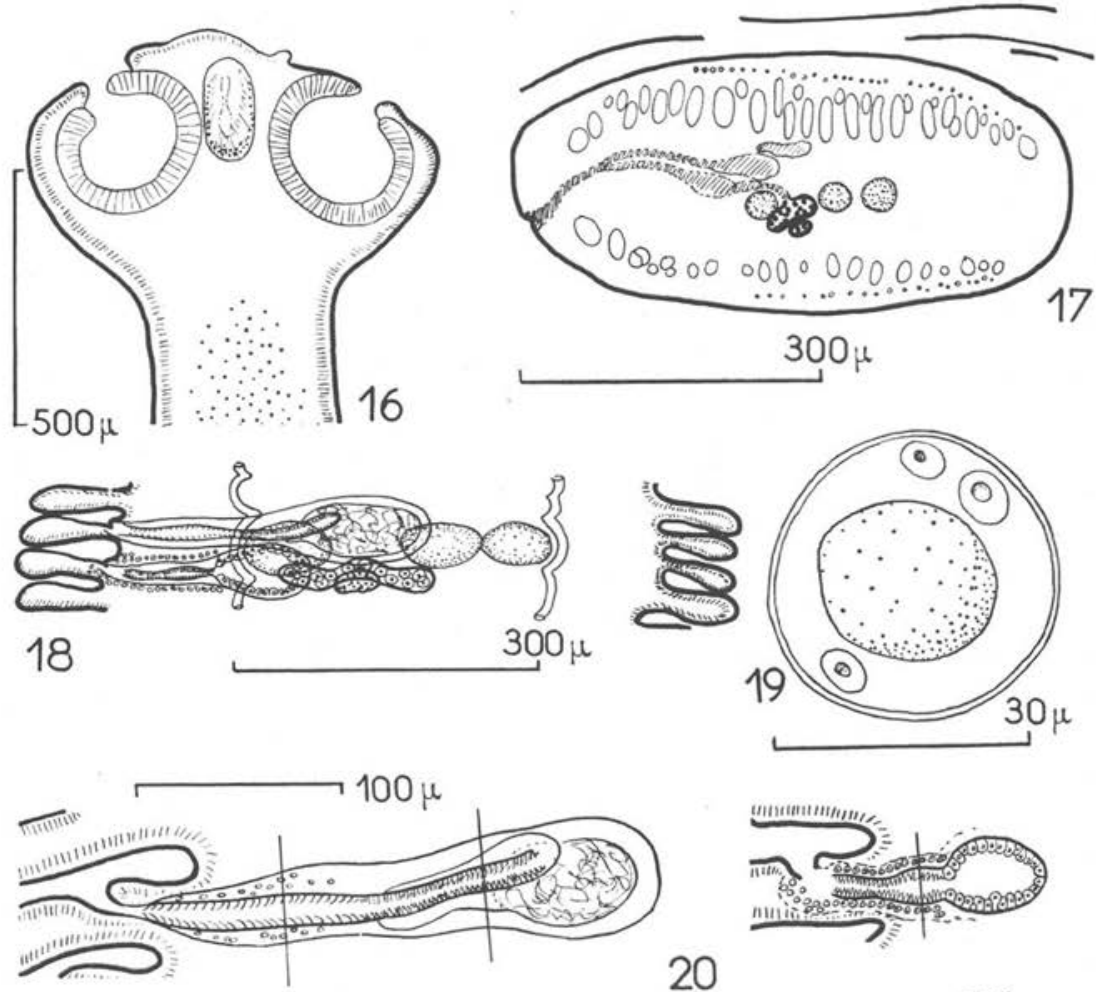

20
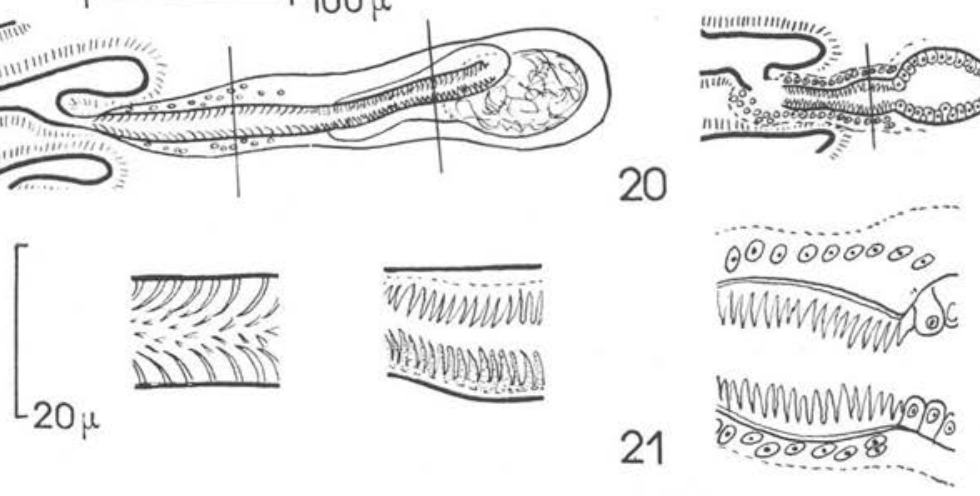

Planche 5. - H. $(H$.$) glandularis Fuhrm., 1909. Types (d'après coupes histologiques).$ 16. Scolex. 17. Anneaux immatures (coupe transversale). 18. Rapport des organes (reconstitution schématique d'anneaux mûrs). 19. CEuf immature. 20. Poche du cirre et vagin. 21. Détail des structures du cirre invaginé, du canal déférent et du vagin (observés au niveau indiqué par les traits). 


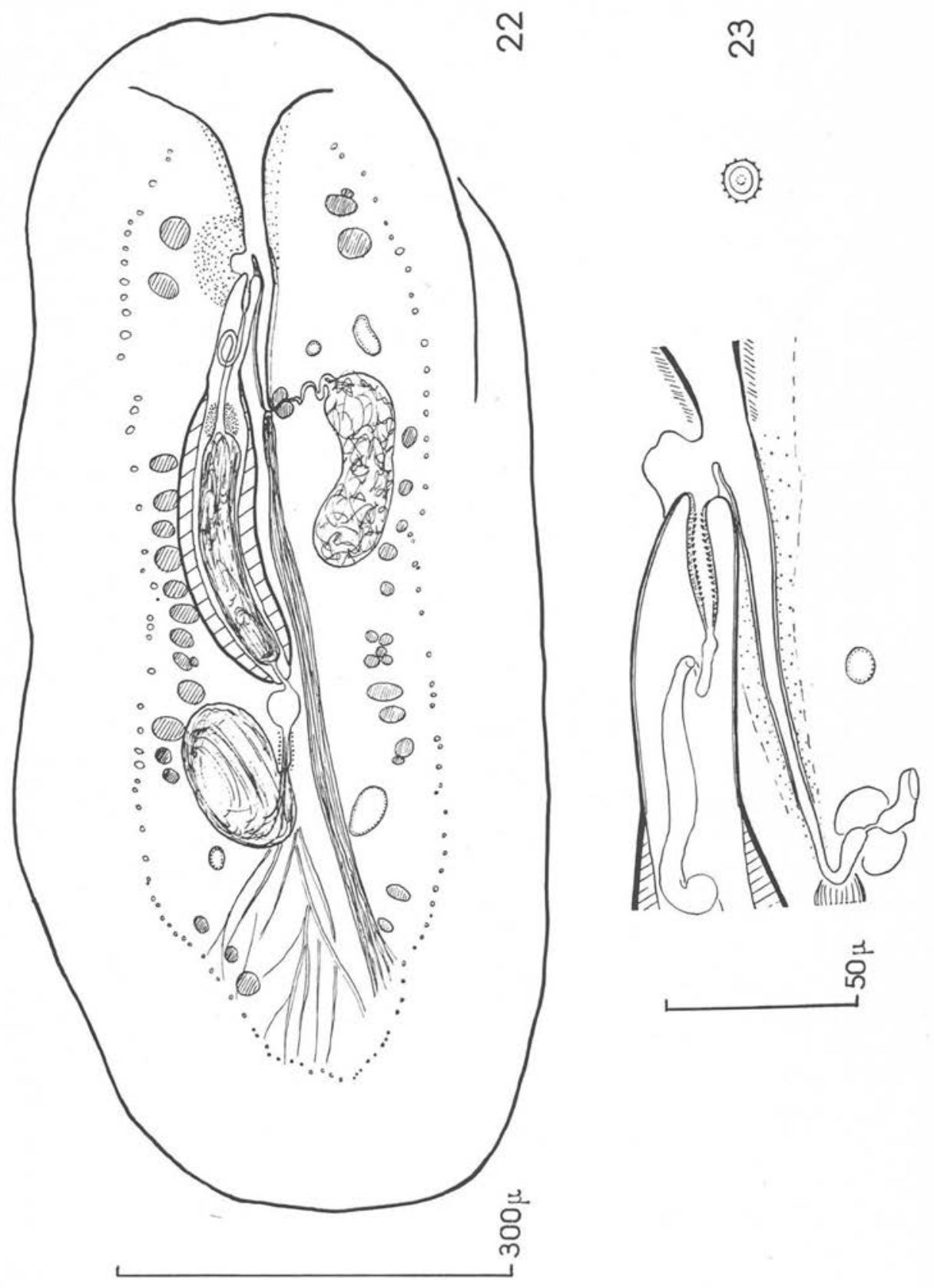

Planche 6. $-H$. (H.) himantopodis (Krabbe, 1869). Types. 22. Coupe d'anneau gravide jeune. 23. Conduits génitaux mâle et femelle. A droite, coupe optique du cirre évaginé. 

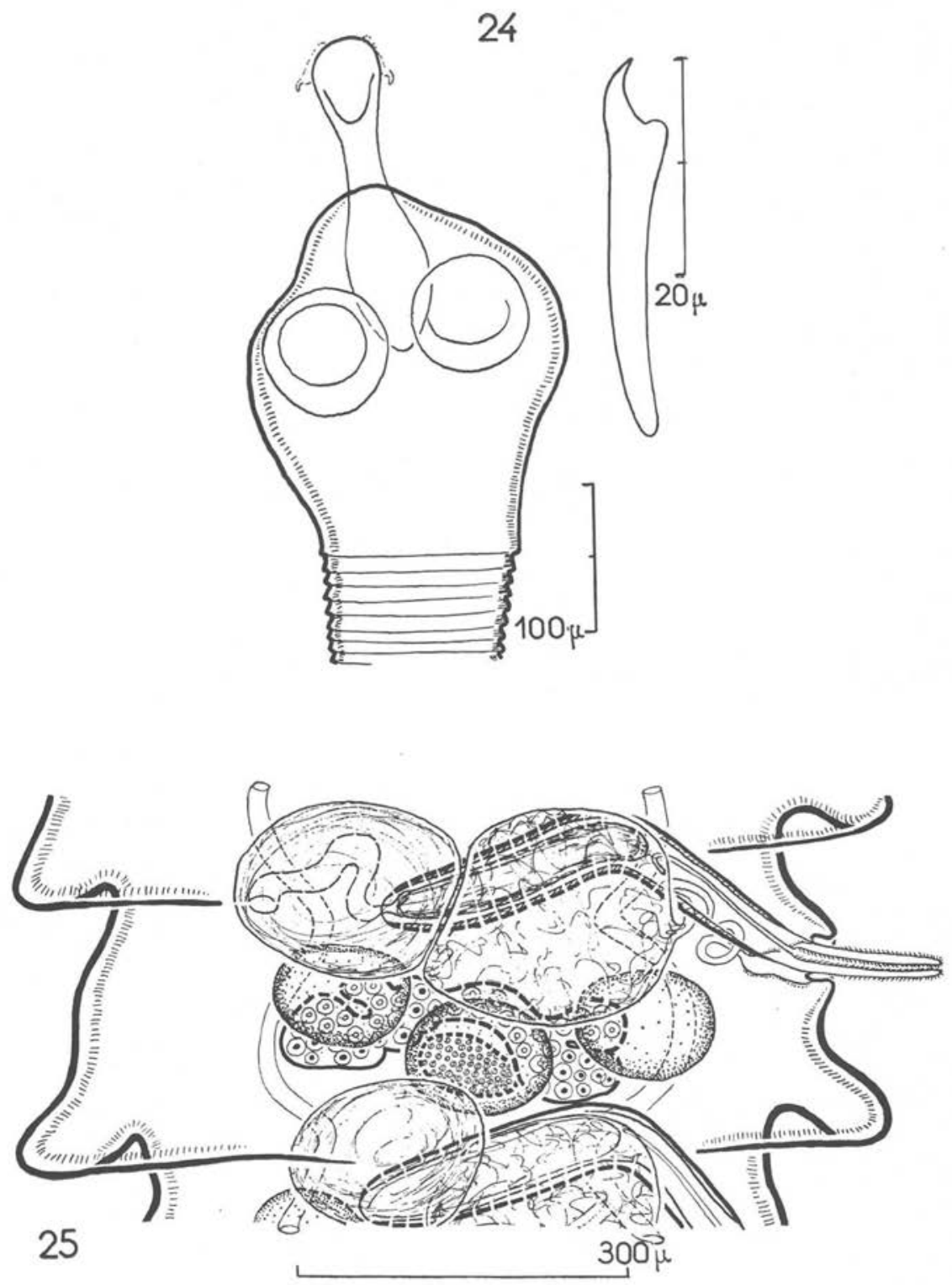

Planche 7. - Légende page suivante. 

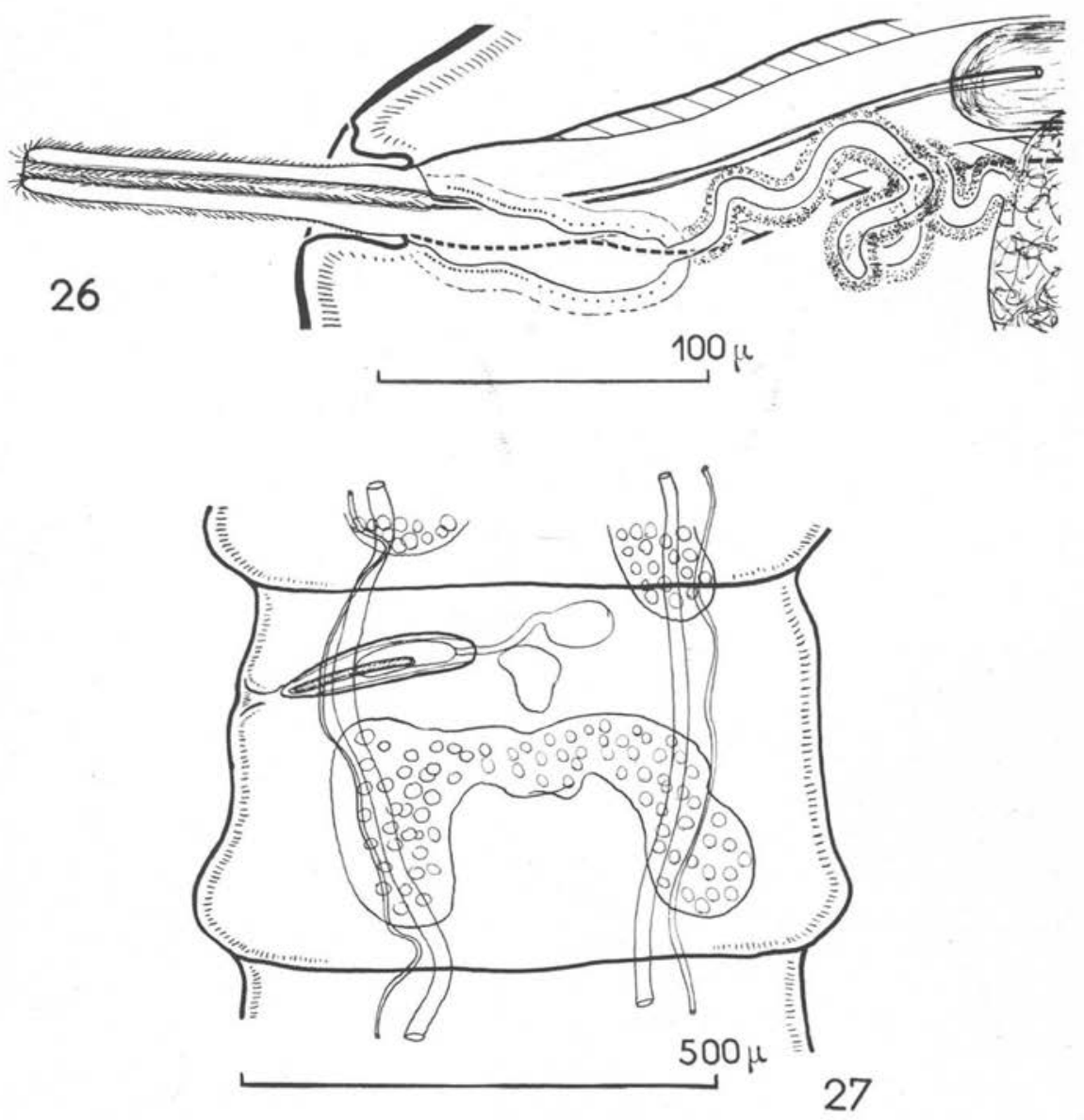

Planche 7. $-H$. (H.) interrupta (Rud., 1802). Exemplaires de Shen Tseng, 1933. 24. Scolex et crochets. 25. Anneau mûr, vue dorsale. 26. Conduits génitaux mâle et femelle. Vue ventrale. 27. Anneau gravide jeune (Exemplaire de Joyeux et Baer, 1928). 

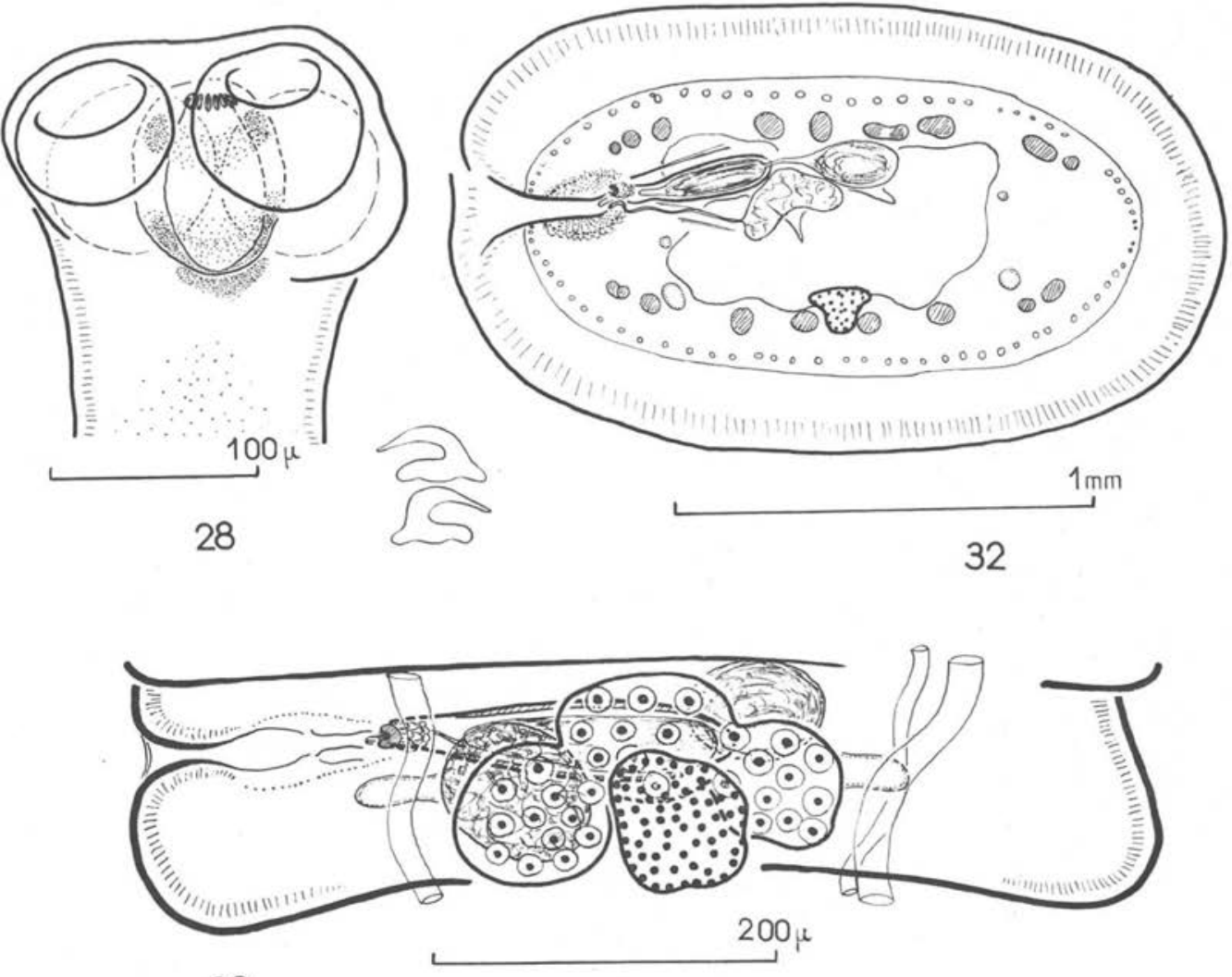

29

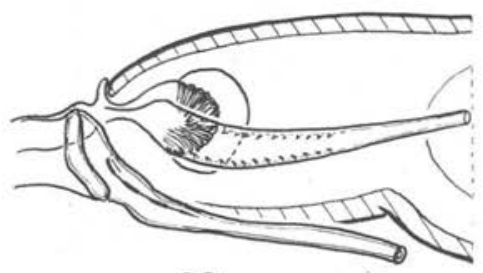

30

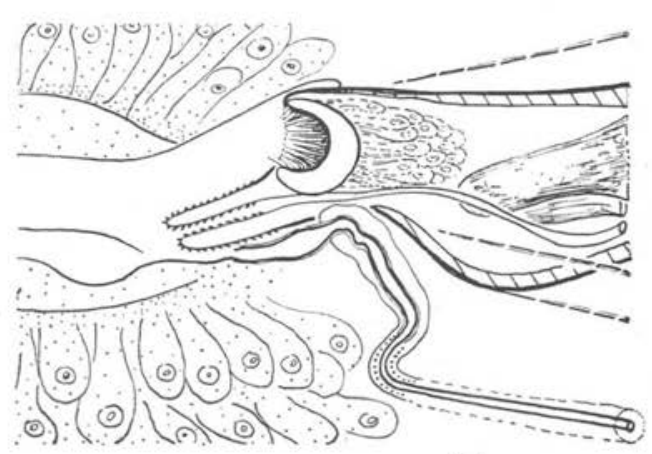

31

Planche 8. - Hym. (H.) kwangensis (Southwell et Lake, 1939). Types. 28. Scolex et crochets. 29. Anneau femelle. Vue ventrale. 30. Conduits génitaux (cirre invaginé). Coupe transversale. 31. Conduits génitaux (cirre partiellement évaginé) et sac accessoire (coupe transversale). 32. Anneau gravide jeune (?). Coupe transversale. 


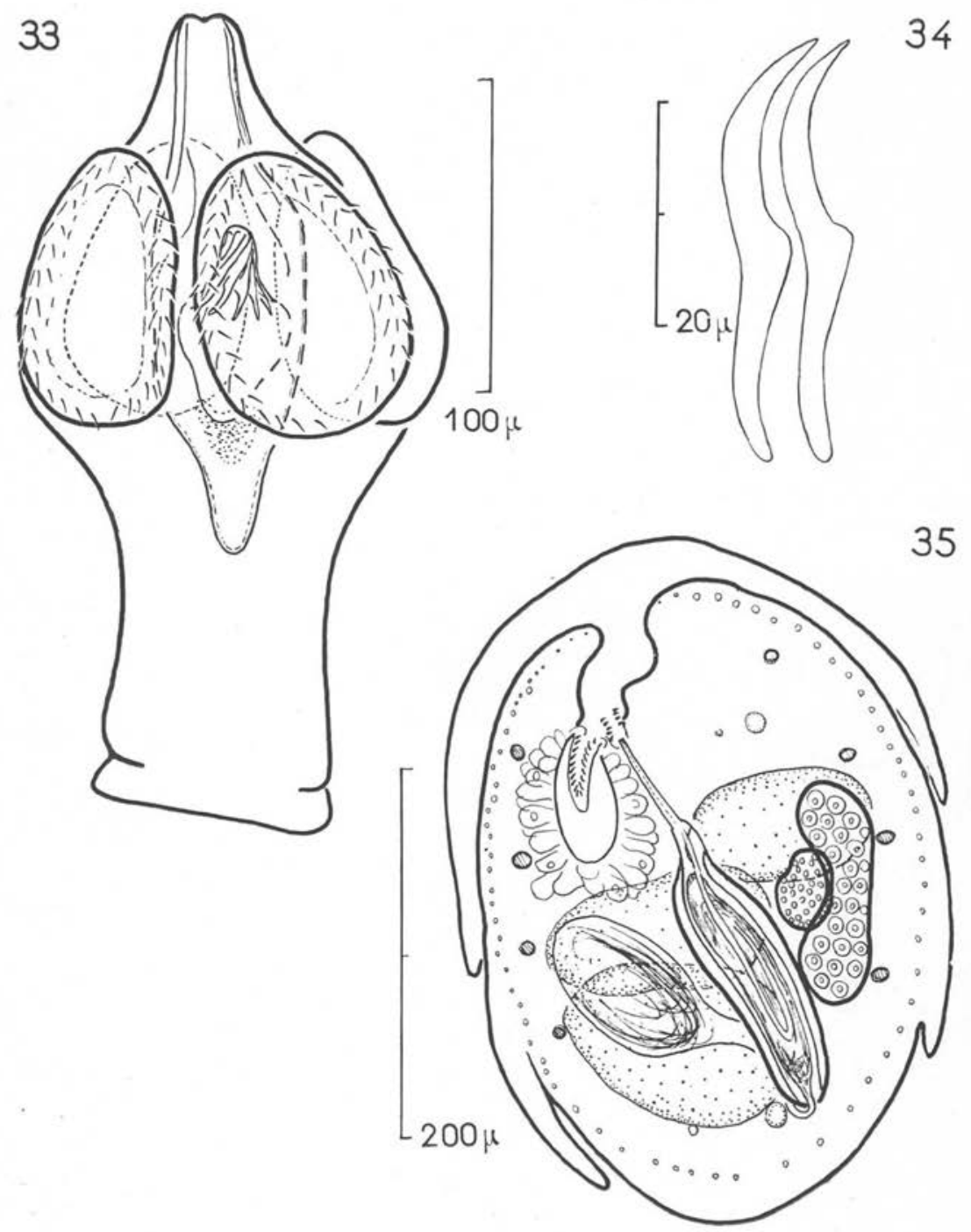

Planche 9. - Légende page suivante. 

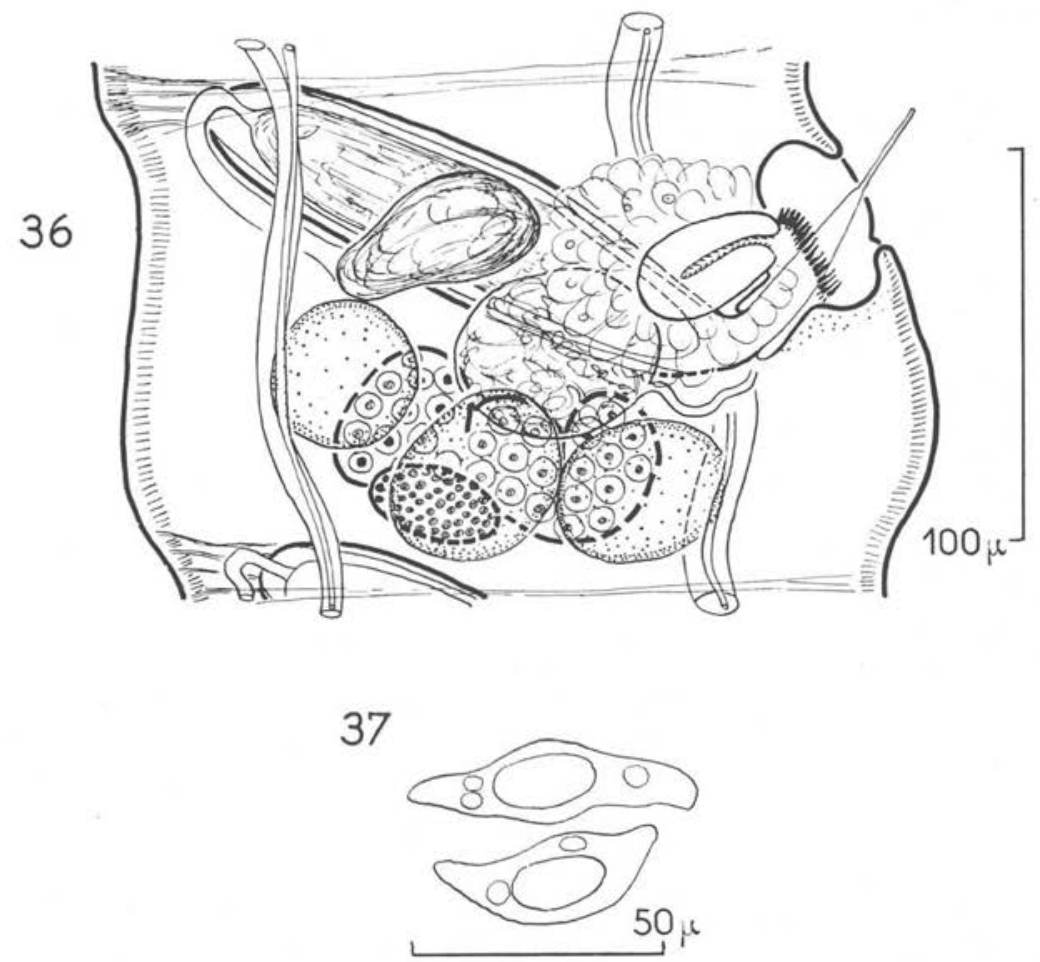

Planche 9. - Hym. (E.) longirostris (Rud., 1819). 33. Scolex (Exemplaire de Meggitt, 1927). 34. Crochets (Exemplaires de Baer et de Meggitt). 35. Coupe transversale d'anneau mûr (exemplaires de Baer, 1956). 36. Anneau mûr (exemplaires de Meggitt, 1927); vue dorsale. 37. CEufs immatures. 


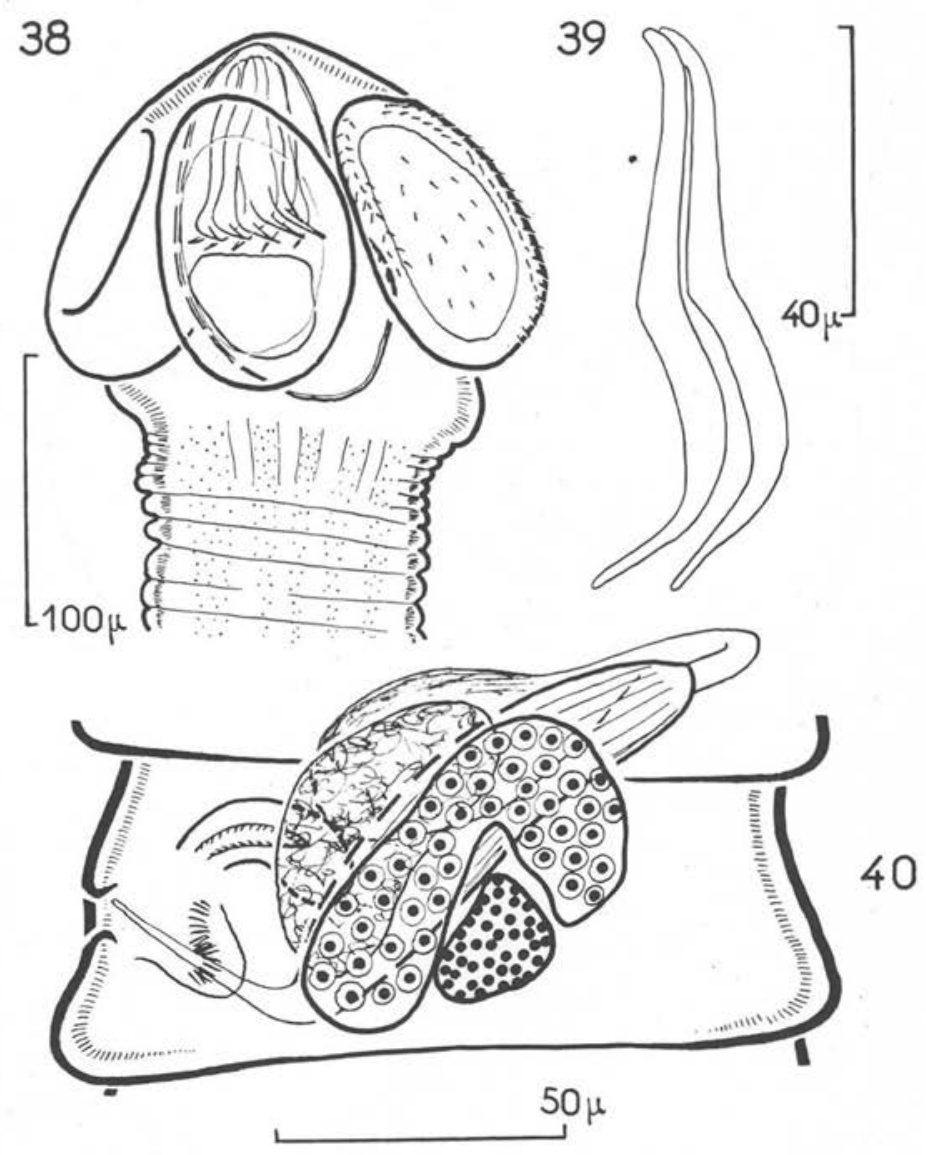

Planche 10. - Hym. (E.) magnisaccis (Meggitt, 1927). Types. 38. Scolex (légèrement écrasé). 39. Crochets. 40. Anneau mûr (vue ventrale). 

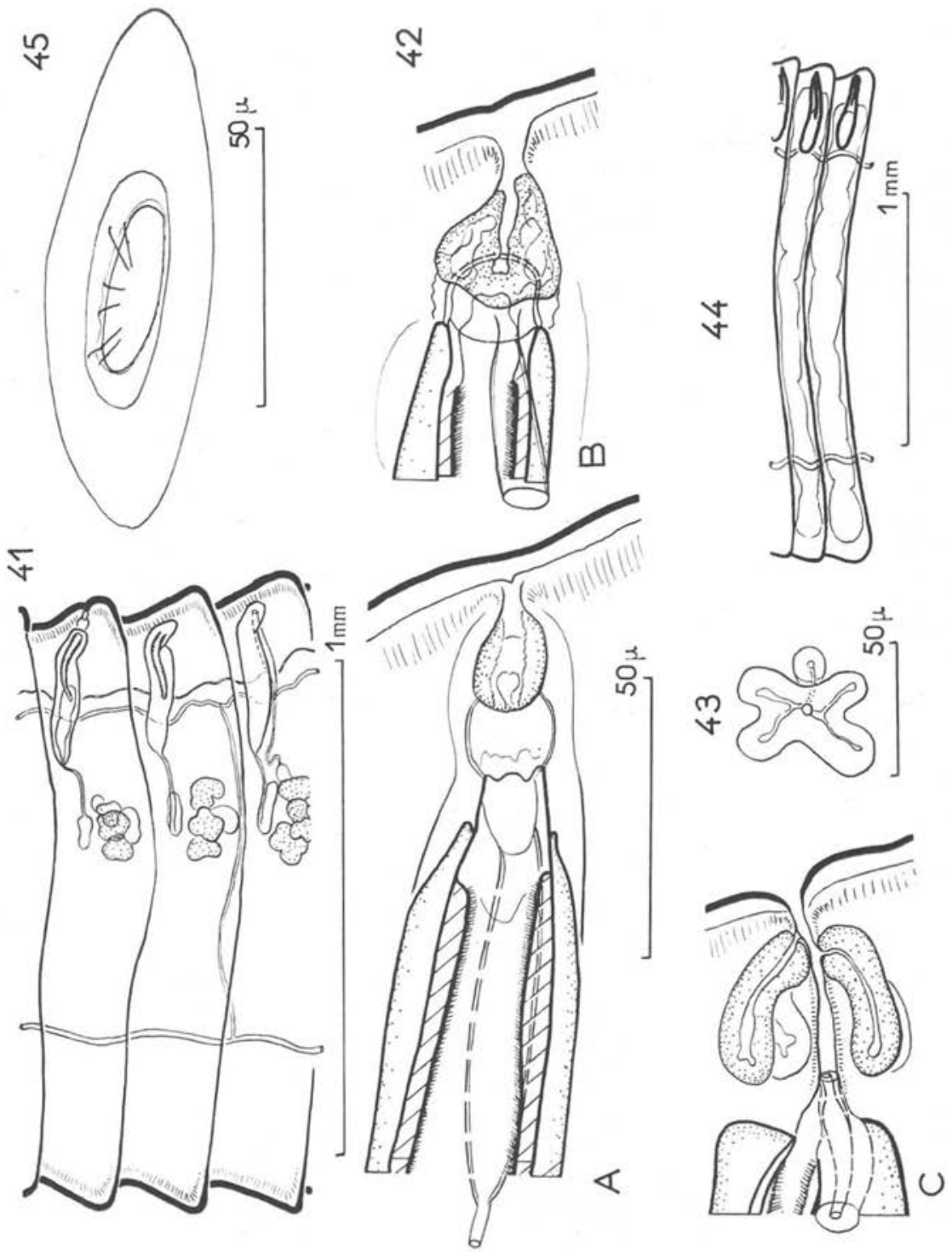

Planche 11. - Hym. (H.) porale (Meggitt, 1927). Types. 41. Anneaux mâles. Vue dorsale. 42. Poche du cirre. A, B, C organogénèse de l'atrium génital (sac accessoire en pointillé). 43. Sac accessoire, vue apicale (à droite, le vagin ventral). 44. Anneau gravide. 45. CEuf. 

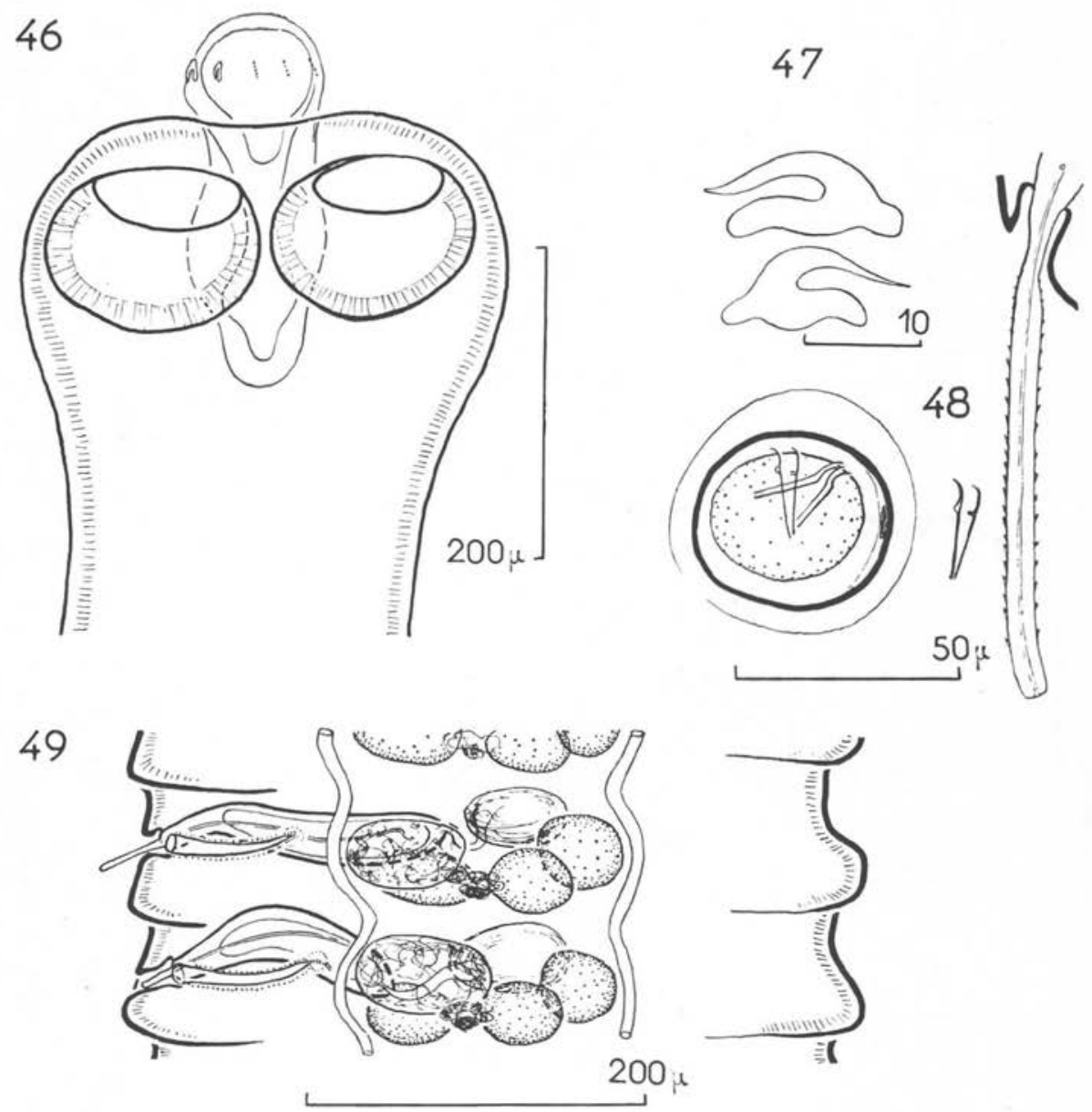

Planche 12. - Légende page suivante. 

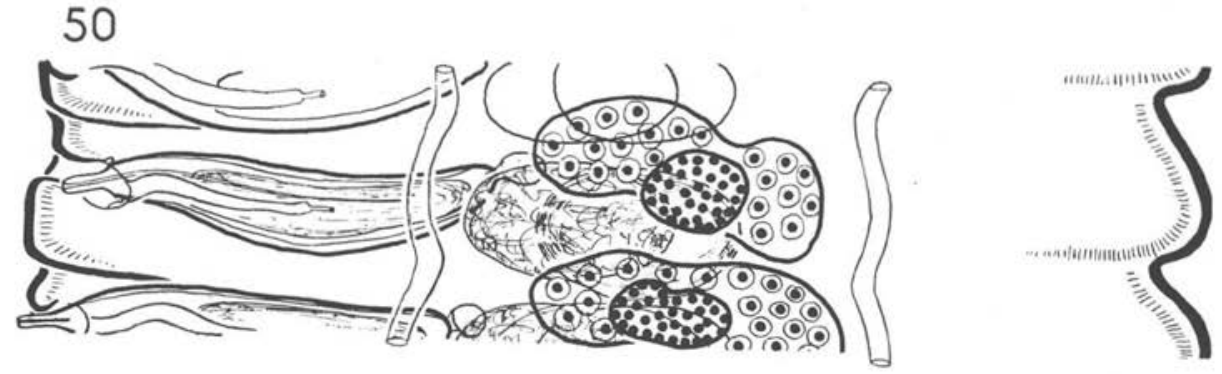

51
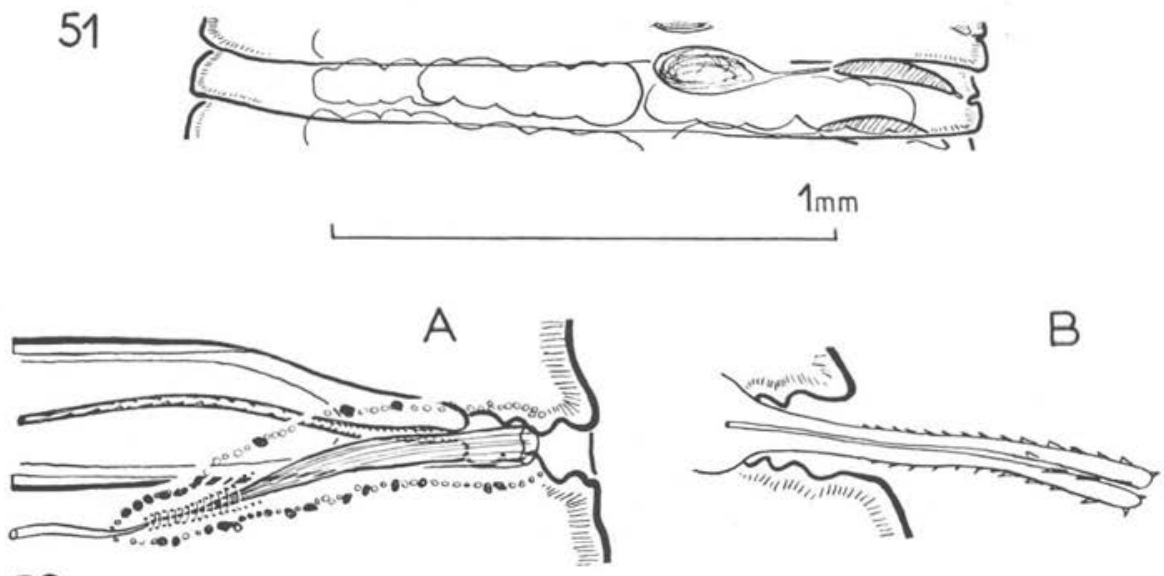

52

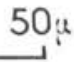

Planche 12. - Hym. (H.) recurvirostrae (Krabbe, 1869). Types (sauf figures 49 et 52). 46. Scolex. 47. Crochets. 48. CEuf mûr et cirre évaginé. 49. Anneau mâle. 50. Anneau femelle. 51. Anneau gravide. 52. Conduits génitaux: a) cirre invaginé et mise en évidence du vagin central ; b) cirre partiellement évaginé. 


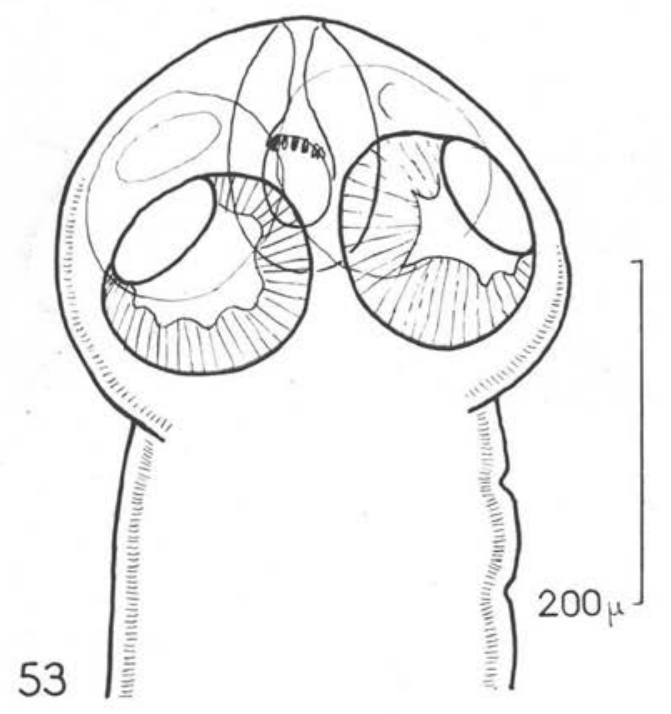

54
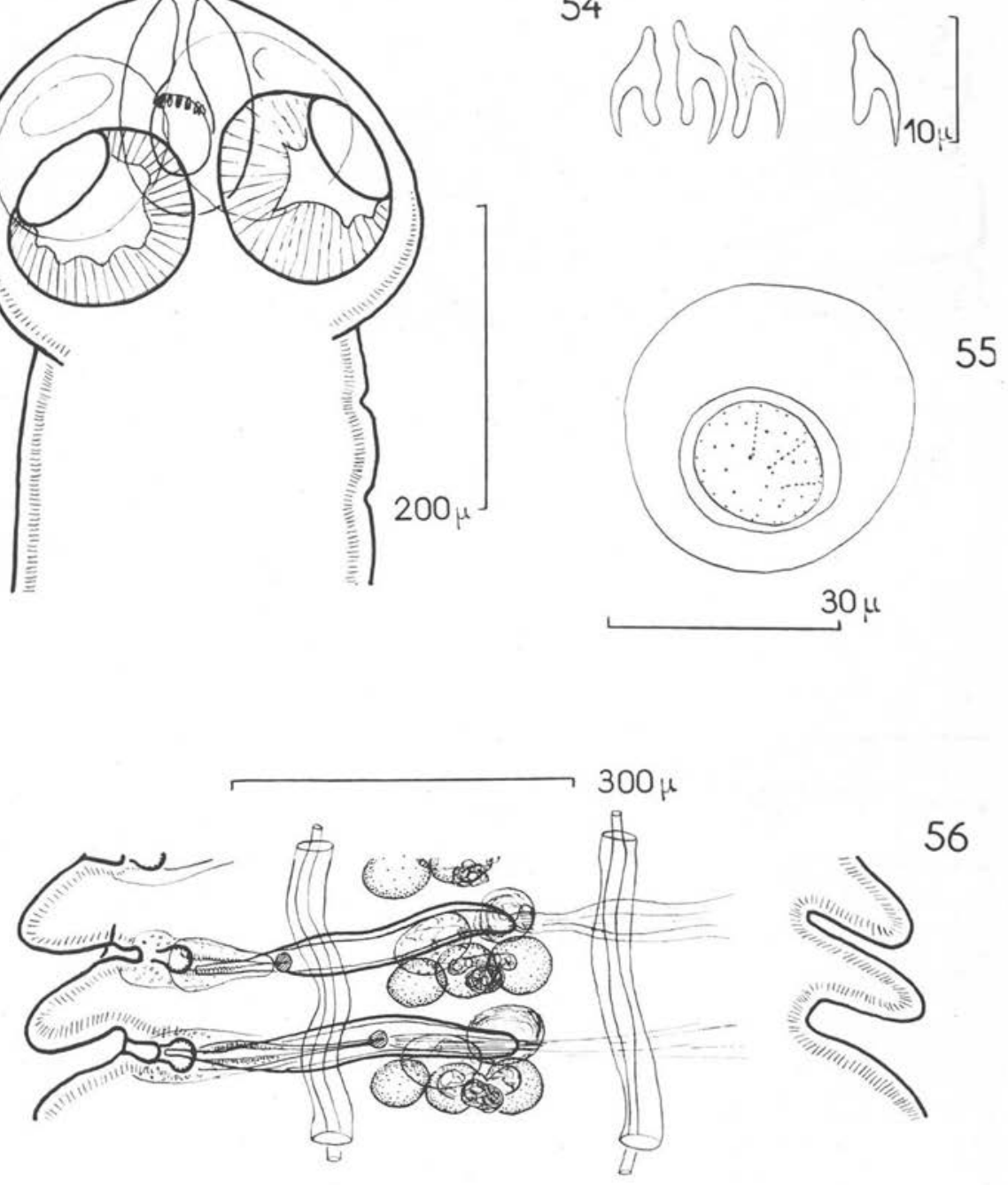

Planche 13. - Légende page suivante. 

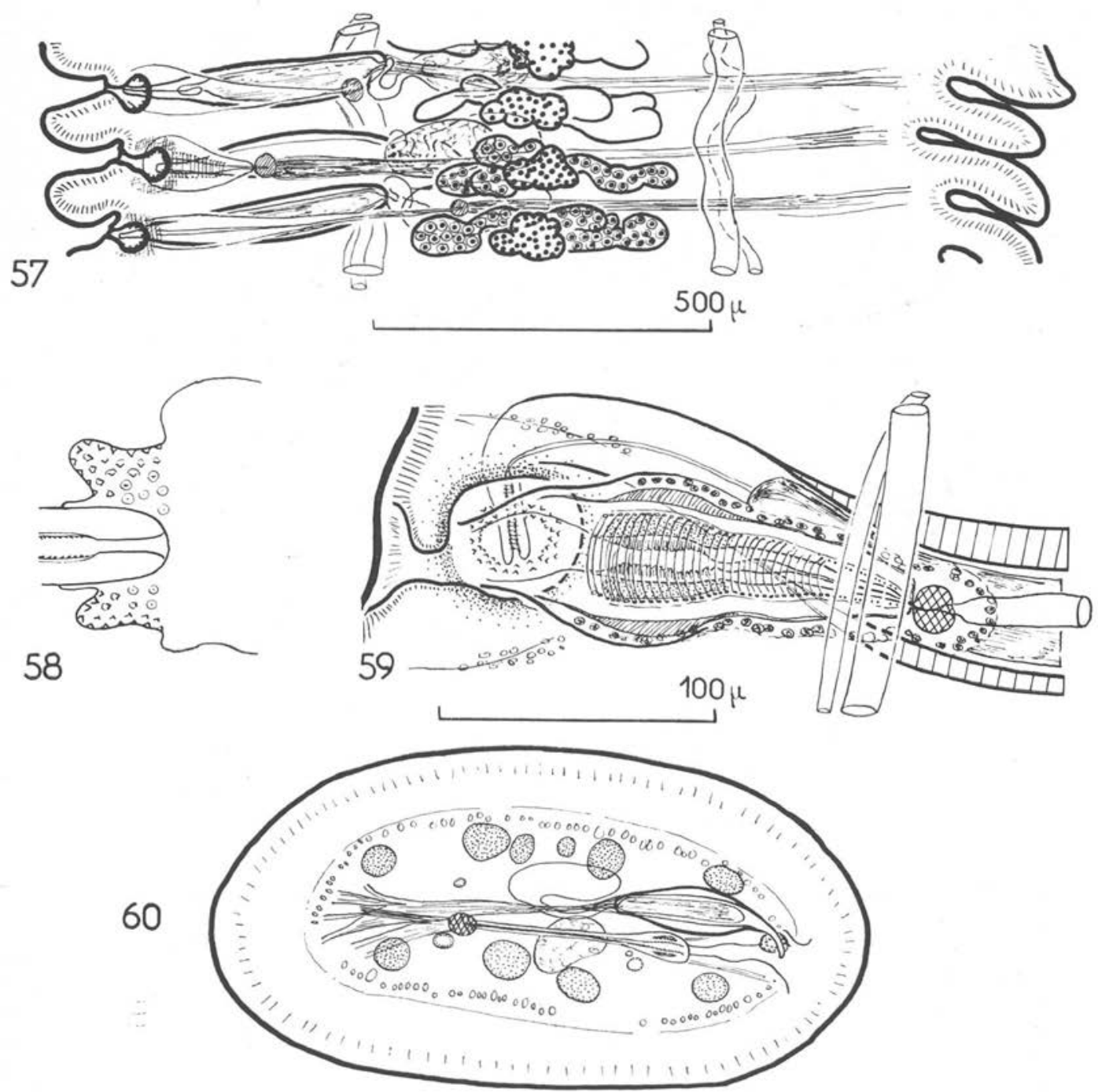

Planche 13. - Hym. (H.) tsengi Joyeux et Baer, 1940. 53. Scolex. 54. Crochets. 55. CEuf immature. 56. Anneaux mâles (vue ventrale). 57. Anneaux femelles (vue ventrale). Dans une tentative de clarification, certains organes ont été omis. 58. Atrium génital schématique. 59. Conduits génitaux et atrium (vue ventrale). 60. Coupe transversale d'un anneau immature. (56-57: exemplaires de Shen Tseng. 53-54-55-58-59: exemplaires de Joyeux. 60 : exemplaires de Joyeux et Baer, 1940). 


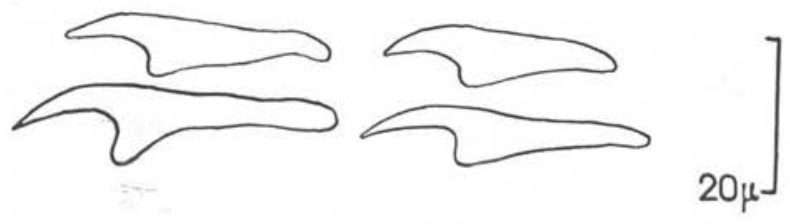

61

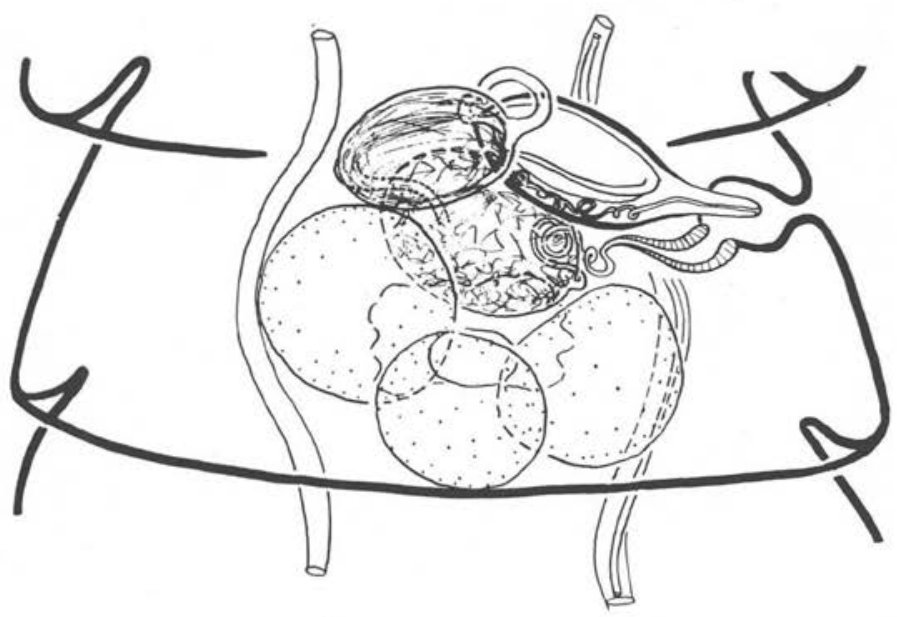

62
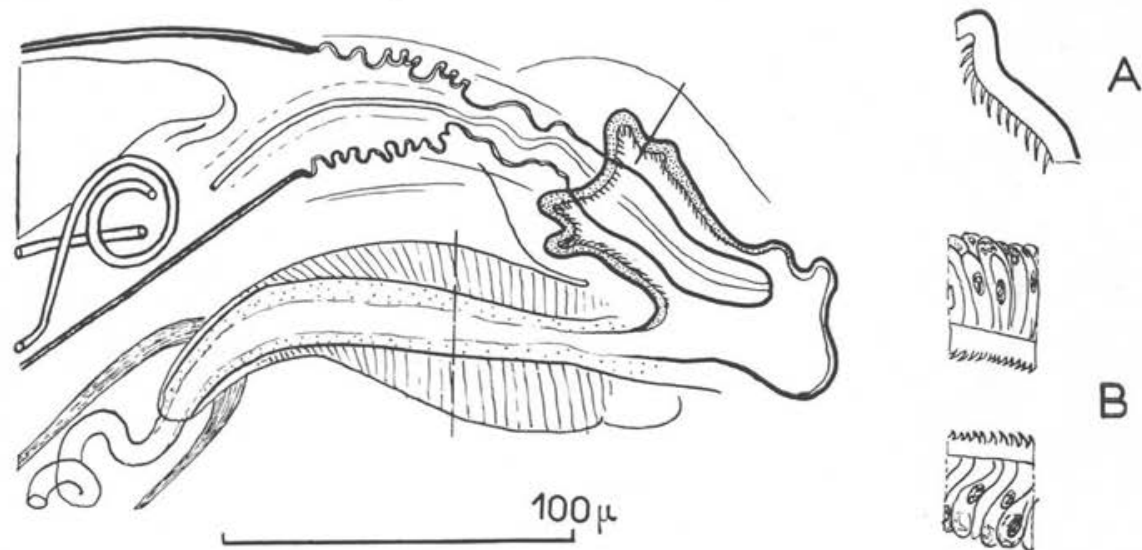

B

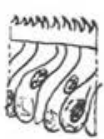

63

Planche 14. - Hymenolepis (Hym.) vaginata Baczynska, 1914. Types. 61. Crochets. 62. Anneau mûr. Vue dorsale (reconstitution schématique d'après coupes histologiques). 63. Conduits génitaux et atrium (coupe histologique). A. Détail de la paroi atriale mâle. B. Détail du vagin. 

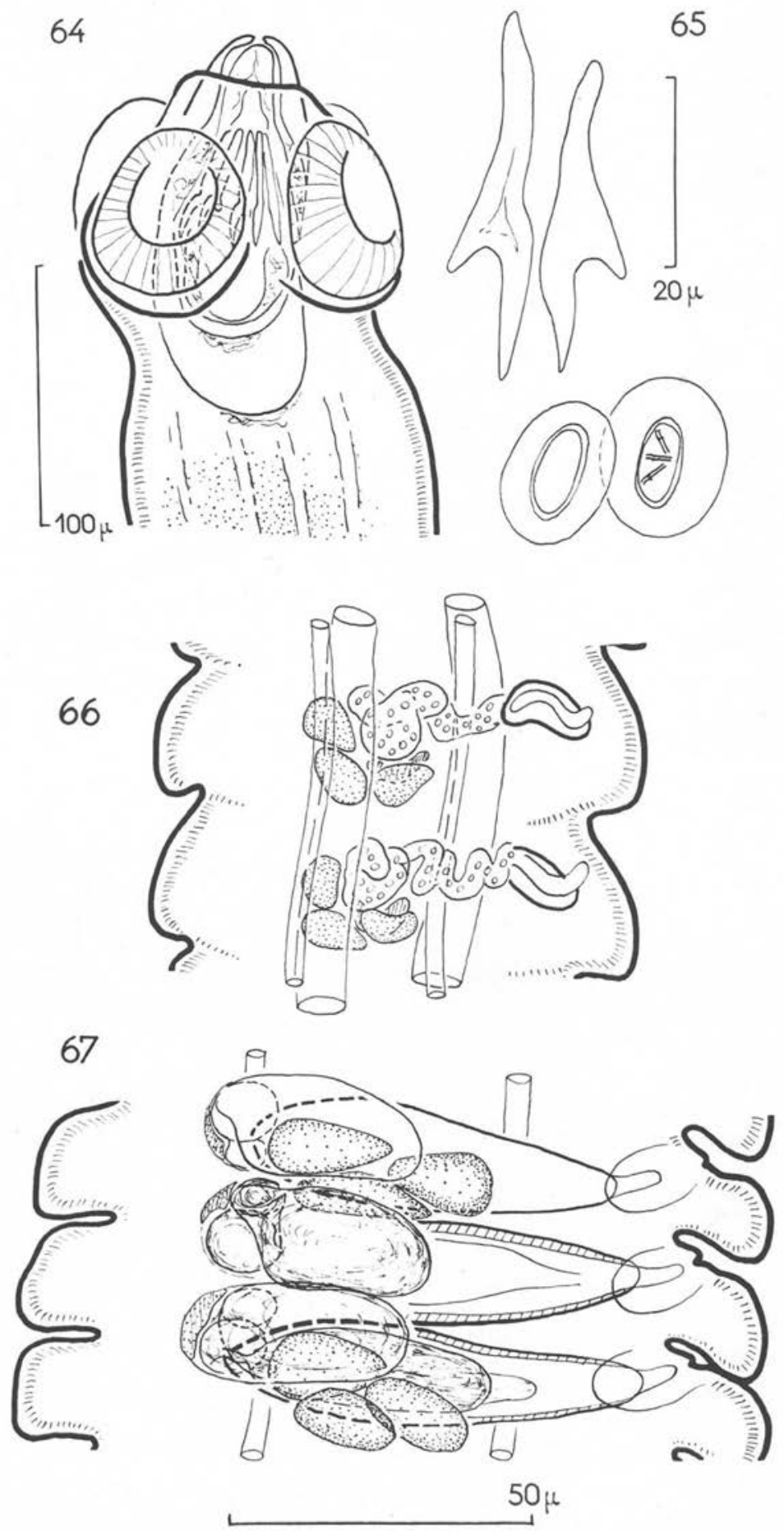

Planche 15. - Légende page suivante. 

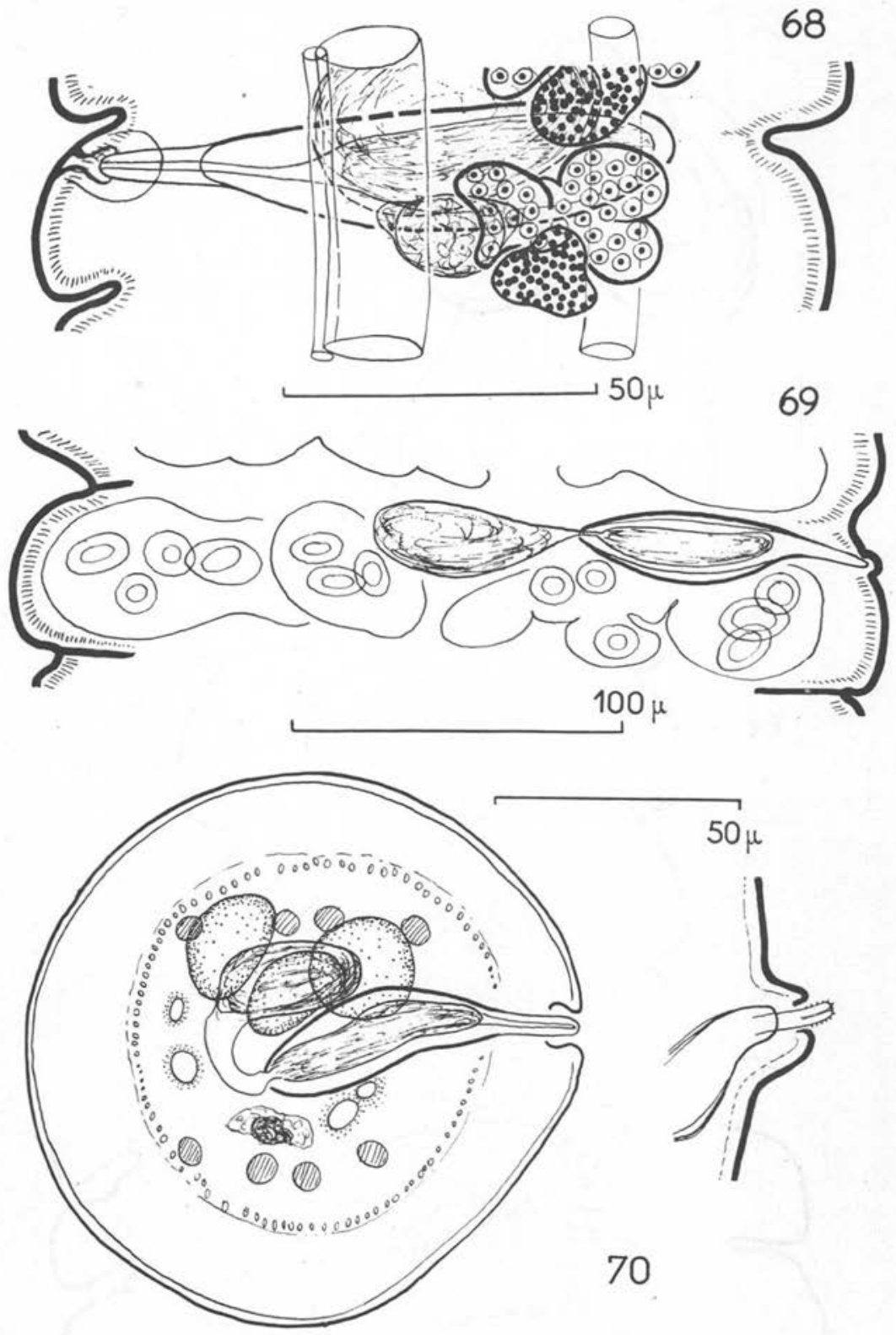

Planche 15. - Hymenolepis (Hym.) aploparaksoïdis n. sp. Types. 64. Scolex. 65. Crochets. CEufs mûrs. 66. Anneaux mâles immatures. 67. Anneaux mâles mûrs. 68. Anneau femelle mûr. 69. Anneau gravide. 70. Coupe transversale d'anneau mâle. Détail d'un cirre évaginé. 


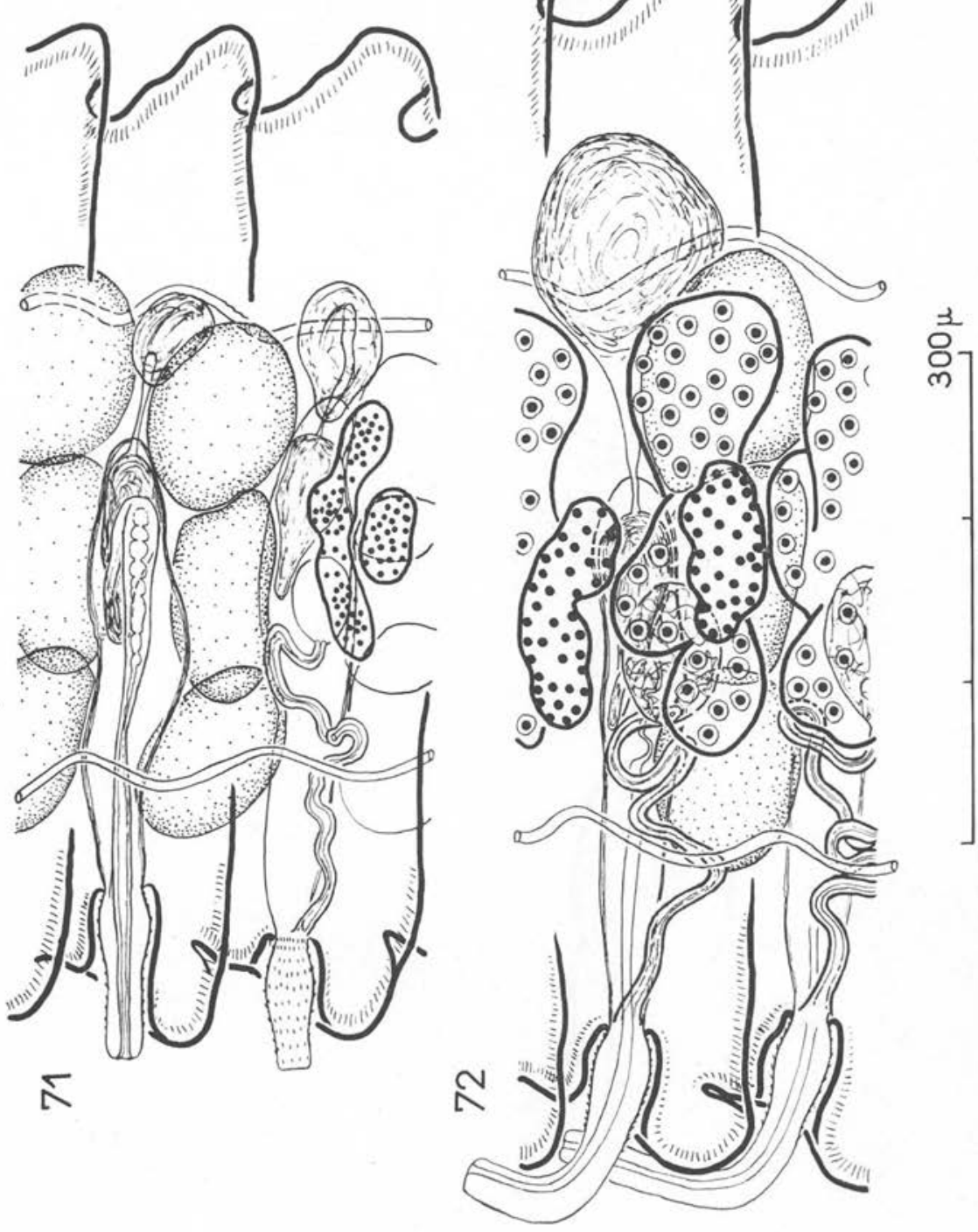

Planche 16. - Légende page suivante. 


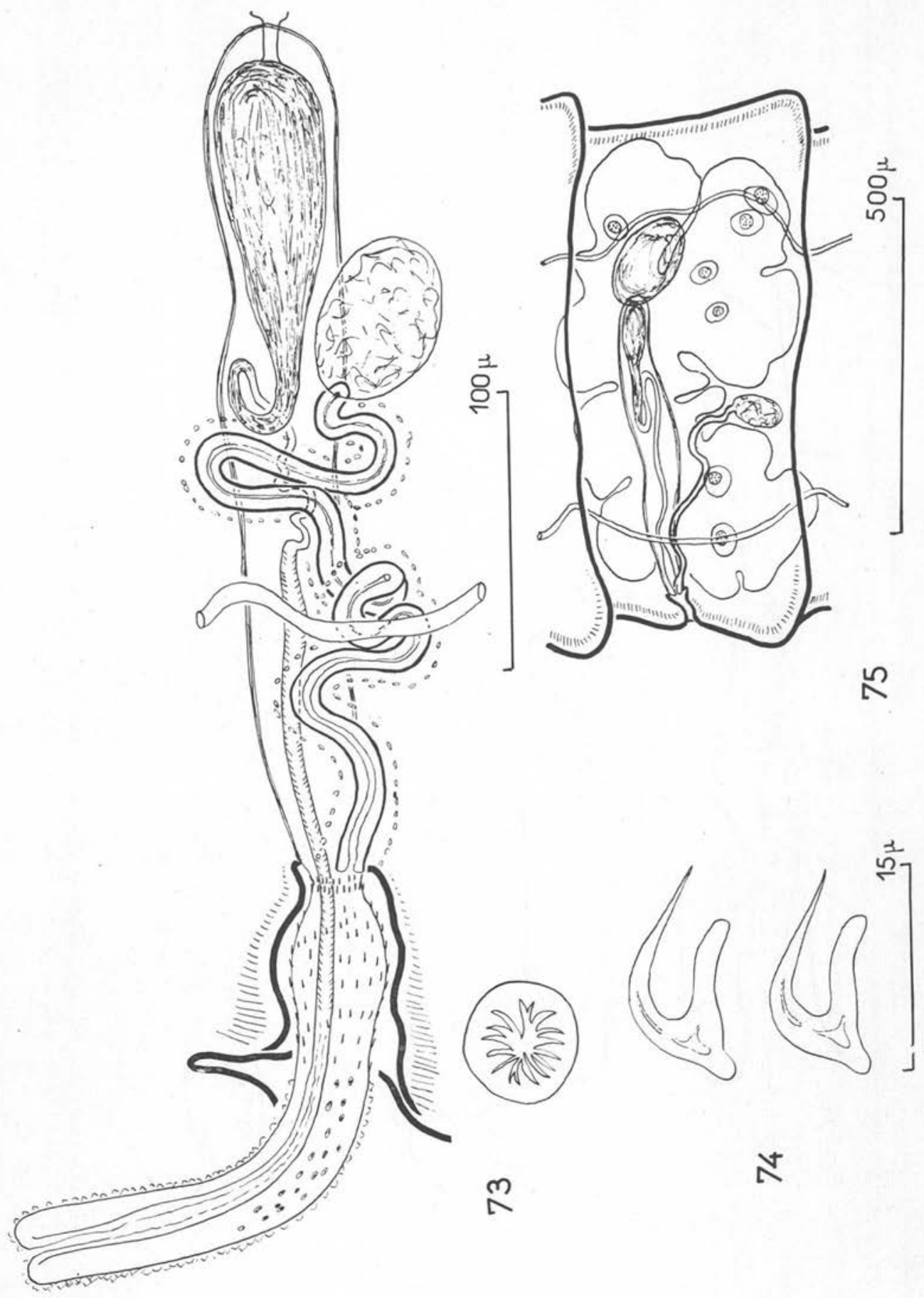

Planche 16. - Hymenolepis (Hym.) rybickae n. sp. Type. 71. Anneaux mâles, vue ventrale; dans l'anneau inférieur, mise en évidence des organes femelles. 72. Anneaux mûrs, hermaphrodites, vue ventrale. 73. Conduits génitaux mâles et femelles et cirre évaginé. Vue ventrale. L'ornementation distale du cirre est incertaine, de même que celle du canal déférent. A gauche, coupe optique du vagin montrant les villosités. 74. Crochets du rostre. 75. Anneau gravide (vue ventrale). 